\title{
Analysis of timekeeper implicates antagonism between CK2 and PP2A during Drosophila neurogenesis
}

\author{
Ezgi Kunttas \\ West Virginia University
}

Follow this and additional works at: https://researchrepository.wvu.edu/etd

\section{Recommended Citation}

Kunttas, Ezgi, "Analysis of timekeeper implicates antagonism between CK2 and PP2A during Drosophila neurogenesis" (2008). Graduate Theses, Dissertations, and Problem Reports. 4393.

https://researchrepository.wvu.edu/etd/4393

This Thesis is protected by copyright and/or related rights. It has been brought to you by the The Research Repository @ WVU with permission from the rights-holder(s). You are free to use this Thesis in any way that is permitted by the copyright and related rights legislation that applies to your use. For other uses you must obtain permission from the rights-holder(s) directly, unless additional rights are indicated by a Creative Commons license in the record and/ or on the work itself. This Thesis has been accepted for inclusion in WVU Graduate Theses, Dissertations, and Problem Reports collection by an authorized administrator of The Research Repository @ WVU. For more information, please contact researchrepository@mail.wvu.edu. 


\title{
ANALYSIS OF TIMEKEEPER IMPLICATES ANTAGONISM BETWEEN CK2 AND PP2A DURING DROSOPHILA NEUROGENESIS
}

\author{
Ezgi Kunttas
}

\author{
Thesis submitted to the \\ Eberly College of Arts and Sciences \\ West Virginia University \\ in partial fulfillment of the requirements \\ for the degree of
}

\author{
Master of Science \\ in \\ Biology
}
Dr Ashok P. Bidwai, Ph.D., Chair Dr Clifton P. Bishop, Ph.D.
Dr Philip E. Keeting, Ph.D.

\section{Department of Biology}
Morgantown, West Virginia 2008

Keywords: CK2, Timekeeper, PP2A, Notch Signaling, D. melanogaster, S. cerevisiae Copyright 2008, Ezgi Kunttas-Tatli 


\title{
Analysis of Timekeeper implicates antagonism between CK2 and PP2A during Drosophila neurogenesis
}

\author{
Ezgi Kunttas
}

\begin{abstract}
It is increasingly becoming apparent that dynamic control of phosphorylation and dephosphorylation underlies the proper spatial and temporal activities of many signaling circuits including Notch $(\mathrm{N})$. Emerging evidence suggests that protein kinase CK2 mediates the effects of $\mathrm{N}$ during Drosophila eye and bristle development via phosphorylation of $\mathrm{E}(\mathrm{spl})$ repressors. Previous studies in our laboratory shown that reducing levels/activity of CK2 elicits rough eyes due to excess $\mathrm{R} 8$ founders and ectopic and split bristles due to excess SOP's, both indicative of impaired lateral inhibition and $\mathrm{N}$ signaling. While these studies implicate a role for $\mathrm{CK} 2$ in the regulation of $\mathrm{E}(\mathrm{spl})$, the identity and/or involvement of a cognate phosphatase remain unknown. A dominant mutation of CK2 $\alpha$, Timekeeper (Tik), harbors two amino acid substitutions, $M^{161} \mathrm{~K}$ and $\mathrm{E}^{165} \mathrm{D}$. The former substitution is thought to predominantly contribute to the dominant behavior of Tik, since it lies in the ATP binding pocket. However, the $E^{165} \mathrm{D}$ mutation might also contribute to the behavior of Tik, given that it resides in a region, which harbors the HEN/HRKL motif, mediates recruitment of the phosphatase PP2A. Therefore, the possibility arises that the analysis of Tik might uncover the relationship between CK2 and PP2A. To test this hypothesis, we first generated two variants of CK2 that harbor either of the Tik substitutions, which were then used in vivo in Drosophila. We find that misexpression of either the MK or the ED variant elicits ectopic bristles whose frequency and penetrance closely approximate that seen with Tik. We have also tested for the ability of these variants to suppress the neural defects of $N^{\text {spl }}$ flies and found that MK and ED suppress the retinal defects of $N^{s p l}$. Then, we have tested the role of PP2A during eye and bristle development in wild type and/or $N^{\text {spl }}$ flies. We find that increasing PP2A dosage (UAS-mts) elicits ectopic and missing bristles in $\mathrm{N}^{+}$flies, both indicative of a loss of $\mathrm{N}$ signaling. In addition, we find that increased dosage of PP2A suppresses the rough and reduced eye phenotypes of $N^{s p l}$ flies, a result that is also associated with decreased CK2 levels/activity. Together, these results suggest that PP2A activity might attenuate, whereas CK2 augments, inhibitory $\mathrm{N}$ signaling. Given the previously described genetic and direct biochemical interactions between CK2 and $\mathrm{E}(\mathrm{spl}) \mathrm{M} 8 / \mathrm{M} 5 / \mathrm{M} 7, \mathrm{PP} 2 \mathrm{~A}$ might serve in an antagonistic manner to CK2.
\end{abstract}




\section{ACKNOWLEDGEMENTS}

It has been almost 5 years since I moved to Morgantown and started a new life and my professional career. These years were among the best years of my career and my life. Therefore, I feel compelled to thank all of those who cared for me throughout these years.

First and foremost, I am grateful to my family in Turkey. My mom Kamuran and my dad Veli made this journey possible for me financially and mentally. I have always felt their love and support through the good and not so good days. You have always been a source of encouragement, trust and inspiration to me. I would also like to thank to my family here, my husband, Emre. During these past years, we had the chance to grow up together, and became each other's everything. I am so lucky that I have found you in Egypt and have the chance to share this life together. Things just aren't right without you. I would also like to thank to my sister, Sima, for her support and love.

Besides from my family, I am also thankful for all the support that I received from my laboratory, without whom this work wouldn't be possible. First, I would like to thank to my research mentor, Dr Ashok Bidwai. Without your vision, experience and expertise, I wouldn't be able to work on this great project. I am glad to have you as my mentor and thankful to him for his guidance throughout these rookie years as a scientist. I would also like to thank my committee members Dr Clifton Bishop and Dr Philip Keeting for their input and suggestions throughout my project. In addition, I like to thank to the other members of my lab; Anasua, Bhaskar, Pallavi, Jee-Eun, Umesh and Sofia. You were always ready to help and made this project easier as well as more enjoyable. I consider myself lucky to be able to spend three years in this nurturing environment.

Lastly, I would express my gratitude to the Department of Biology, specifically to Dr Cumming and Dr Wells, for the facilities they provided. In addition, I would like to thank the staff, Marleen, Pat and Wendy, for their kindness and support. 


\begin{tabular}{|c|c|}
\hline Table of Contents & Page \\
\hline Abstract & ii \\
\hline Acknowledgements & iii \\
\hline Table of Contents & Iv \\
\hline List of Figures & v-vi \\
\hline List of Tables & vii \\
\hline Abbreviations & viii-ix \\
\hline $\begin{array}{l}\text { Chapter } 1 \\
\text { Introduction }\end{array}$ & $1-56$ \\
\hline $\begin{array}{l}\text { Chapter } 2 \\
\text { The dominant effects of Tik during eye and bristle development appear to be } \\
\text { a consequence of a 'double hit' }\end{array}$ & $57-103$ \\
\hline $\begin{array}{l}\text { Chapter } 3 \\
\text { Potential antagonistic roles of CK2 and PP2A in Notch Signaling in } \\
\text { Drosophila }\end{array}$ & $104-112$ \\
\hline $\begin{array}{l}\text { Chapter } 4 \\
\text { Conclusions }\end{array}$ & $113-116$ \\
\hline $\begin{array}{l}\text { Chapter } 5 \\
\text { References }\end{array}$ & $117-127$ \\
\hline Appendix A & 128 \\
\hline
\end{tabular}




\begin{tabular}{|c|c|}
\hline List of Figures & Page \\
\hline $\begin{array}{l}\text { Figure 1: Structure of CK2 holoenzyme and activity comparison of CK2 with PKA } \\
\text { and CDK. }\end{array}$ & 6 \\
\hline Figure 2: Alignment of CK2 $\alpha$ subunits. & 9 \\
\hline Figure 3: Functional domains of $\mathrm{CK} 2 \alpha$ and $\mathrm{CK} 2 \beta$ : & 11 \\
\hline Figure 4: Alignment of CK2 $\beta$ subunits. & 13 \\
\hline Figure 5: Structure of CK2 $\beta$ autophosphorylation and Zinc binding domains. & 15 \\
\hline Figure 6: Structures of CK2 alleles. & 18 \\
\hline Figure 7: Global roles of CK2. & 20 \\
\hline Figure 8: Regulation of CK2. & 25 \\
\hline Figure 9: CK2 interacting protein-1 (CKIP-1) and HIKE motif. & 28 \\
\hline Figure 10: Amino acid substitutions in Tik. & 32 \\
\hline Figure 11: Structure of PP2A holoenzyme. & 37 \\
\hline Figure 12: Alignment of PP2A catalytic subunits. & 39 \\
\hline Figure 13: Global roles of PP2A. & 42 \\
\hline Figure 14: The Enhancer of split Complex. & 49 \\
\hline Figure 15: Notch mediated Lateral Inhibition. & 51 \\
\hline Figure 16: Role of Lateral Inhibition during bristle and eye morphogenesis. & 53 \\
\hline Figure 17: Analysis of dropout clones. & 65 \\
\hline Figure 18: Mating type two-hybrid assay. & 68 \\
\hline Figure 19: The Gal4-UAS system in Drosophila. & 72 \\
\hline Figure 20: The schematic of constructs & 75 \\
\hline Figure 21: Structural comparison between $\mathrm{M}^{161} \mathrm{~K}$ and $\mathrm{K}^{66} \mathrm{M}$ substitutions. & 77 \\
\hline Figure 22: Analysis in yeast rescued by Ts alleles of yCK2 $\alpha$. & 80 \\
\hline Figure 23: Analysis in yeast rescued by Ts alleles of dCK2 $\alpha$. & 82 \\
\hline $\begin{array}{l}\text { Figure 24: The mean generation time and terminal phenotypes of strains rescued } \\
\text { by dCK } 2 \alpha-M K \text { and } d C K 2 \alpha-E D\end{array}$ & 84 \\
\hline Figure 25: Expression and stability of dCK2 $\alpha$ constructs. & 87 \\
\hline Figure 26: Interaction of dCK2 $\alpha$ variants with CK2 $\beta$. & 90 \\
\hline Figure 27: Mapping and balancing of the transgenic lines. & 93 \\
\hline
\end{tabular}




\begin{tabular}{|c|c|}
\hline $\begin{array}{l}\text { Figure 28: Ability of UAS-CK2 } \alpha-M K \text { and UAS-CK2 } \alpha-E D \text { constructs to elicit bristle } \\
\text { abnormalities. }\end{array}$ & 96 \\
\hline $\begin{array}{l}\text { Figure 29: Ability of } U A S-C K 2 \alpha-M K \text { and } U A S-C K 2 \alpha-E D \text { constructs to elicit bristle } \\
\text { abnormalities. }\end{array}$ & 98 \\
\hline $\begin{array}{l}\text { Figure 30: Ability of UAS-CK2 } \alpha-M K \text { and UAS-CK } 2 \alpha-E D \text { constructs to suppress the } \\
\text { eye phenotype of } N^{s p l} \text {. }\end{array}$ & 100 \\
\hline $\begin{array}{l}\text { Figure 31: Ability of UAS-mtsRNAi and UAS-mtsDN constructs to elicit bristle } \\
\text { abnormalities and comparison to UAS-Tik. }\end{array}$ & 107 \\
\hline $\begin{array}{l}\text { Figure 32: Ability of UAS-mts construct to elicit bristle abnormalities and } \\
\text { comparison to UAS-Tik. }\end{array}$ & 109 \\
\hline Figure 33: Ability of UAS-mts construct to elicit eye defects. & 111 \\
\hline $\begin{array}{l}\text { Figure 34: Ability of UAS-mts construct to suppress the eye phenotype of } N^{s p} I \text {, and } \\
\text { comparison to UAS-CK2 } \alpha-M K \text {. }\end{array}$ & 112 \\
\hline Figure 35: Potential antagonistic roles of $\mathrm{CK} 2$ and PP2A during $\mathrm{N}$ signaling. & 116 \\
\hline
\end{tabular}




\begin{tabular}{|l|l|}
\hline List of Tables & Page \\
\hline Table 1: S. cerevisiae strains & 62 \\
\hline Table 2: Temperature sensitivity of S. cerevisiae strains & 64 \\
\hline Table 3: Primers used during this study & 128 \\
\hline
\end{tabular}




\section{ABBREVIATIONS}

AD-fusion: Activation domain fusion

AMPPNP: 5'-adenylyl-beta,gamma-imidodiphosphate

And: Andante

$\operatorname{Arg}(\mathrm{R})$ : Arginine

Ato: Atonal

ATP: Adenosine Triphosphate

bHLH: basic-helix-loop-helix

cAmp: Cyclic AMP

CADASIL: cerebral autosomal dominant arteriopathy with subcortical infarcts and leukoencephalopathy

CDK: Cyclin dependent kinase

CKIP-1: CK2 interacting protein 1

DB-fusion: DNA binding domain fusion

DI: Delta

dCK2: Drosophila CK2

Dm: Drosophila melanogaster

Dsh: Dishevelled

ED: $d C K 2 \alpha-E^{165} D$

EDTA: Ethylene-diamine-tetra-acetate

EGFR: Epidermal growth factor receptor

$E(S p l) C$ : Enhancer of Split complex

Frz: Frizzled

Glu (E): Glutaimic acid

GOF: Gain of function

GTP: Guanosine Triphosphate

HEAT: are Huntington's disease associated protein (Huntington), elongation factor 3 (EF3),

A subunit of PP2A and a lipid kinase TOR1 lle (I): Isoleucine

$\mathrm{KM}: \mathrm{dCK} 2 \alpha-\mathrm{K}^{66} \mathrm{M}$

LB: Luria Broth

LOF: Loss of function

Lys (K): Lysine

MAPK: Mitogen Activated Protein Kinases

Met (M): Methionine

MF: Morphogenetic furrow

MK: dCK $2 \alpha-M^{161} \mathrm{~K}$

mts: mictortubulestar, catalytic subunit of PP2A

NPT: Non-permissive temperature

N: Notch

$N^{\text {spl }}$ : Notch split

OA: Okadaik acid

ONPG: O-Nitrophenyl_-D-Galactopyranoside

ORF: Open Reading Frame

PP2A: Protein phosphatase 2A

PCR: Polymerase Chain Reaction

PER: Period

PKA: cAMP-dependent protein kinase

PNC: Proneural clusters

PT: Permissive temperature

Ser: Serine 
SDS-PAGE: Sodium dodecyl sulphate - Polyacrylamide gel electrophoresis

SOP: Sensory organ precursor

$\mathrm{Su}(\mathrm{H})$ : Suppressor of Hairless

Thr (T): Threonine

Tik: Timekeeper

TikR: Timekeeper Revertant

TIM: Timeless

Ts: Temperature sensitive

Tyr (Y): Tyrosine

UAS: Upstream Activating Sequence

Y2H: Yeast two hybrid

yCK2: Yeast CK2

yCKA1/2: Yeast CKA1 and CKA2 genes

YPD: Rich glucose medium

YPG: Rich galactose medium

$W^{+}:$mini-white gene

WT: Wild type 
CHAPTER-1.

INTRODUCTION 
During the development of an organism, a fertilized egg (zygote) gives rise to number of cells that form the complex and diverse multicellular structures in the adult organism. This raises numerous questions; such as how equipotent cells become different from each other and how they form organs with positional specificity and complexity? This complexity is regulated through intricate array of cell-cell communications, collectively termed as signal transduction. Signal transduction is a process by which external stimuli are converted into signals within the cell. These signals lead to a variety of changes, such as the regulation of gene expression, cell division/differentiation, altered cellular morphology, cell migration, cell-cell contacts or cellular metabolism. Although there are multiple mechanisms of signal transduction, analyses in various model systems have uncovered an astonishing conservation of the components that define these signaling circuits. It is becoming increasingly apparent that a dynamic control of these communication pathways underlies the proper spatial and temporal activities of these signaling circuits. One of the means by which this dynamic control is conferred upon a signaling pathway is the posttranslational modification of proteins i.e., phosphorylation or dephosphorylation. One third of all cellular proteins are regulated via reversible phosphorylation, which is achieved, by the opposing actions of protein kinases and phosphatases (reviewed in (Glover, 1998)). During these processes, specific residues of target proteins are phosphorylated by protein kinases and these phosphates can be removed by protein phosphatases. This mechanism is especially involved in signal transduction pathways in which multiple proteins become incorporated into complexes that transmit information and control such cellular events such as cell cycle progression, gene expression, apoptosis, and cytoskeletal organization.

\section{HISTORY OF THE PROTEIN KINASE SUPERFAMILY}

The addition of a phosphate group to a protein molecule had been known since the $19^{\text {th }}$ century; however, the function was believed at that time to be of some nutritional 
significance since phosphorous was known to be essential for our diet (Rimington, 1926). In 1954, the laboratory of Eugene Kennedy was the first to describe a kinase following partial purifications of an enzyme from rat liver homogenates, which they named "protein phosphokinase" (Burnett and Kennedy, 1954). They discovered that this enzyme was able to add a phosphate group onto certain serine residues of the milk protein casein, using ATP as a phosphate donor. The significance of kinases and protein phosphorylation remained unclear until cAMP-dependent protein kinase $A(P K A)$ was described by Edwin Krebs and Edmond Fischer in 1955 (reviewed in (Krebs, 1986; Krebs, 1959)). They identified PKA while studying glycogen metabolism and the activation of the enzyme, glycogen phosphorylase. They found that glycogen phosphorylase was subject to reversible phosphorylation and that its phosphorylation status influenced its activity. This was the first evidence that phosphorylation regulated enzymatic activity and these pioneering studies have now led to the discovery of hundreds of additional kinases.

The eukaryotic protein kinases make up one of the largest superfamilies of homologous proteins and are involved in regulating virtually every aspect of organismal biology (Hunter, 1995). Although these enzymes have different structures, are involved in diverse cellular processes, and recognize a wide range of substrates, nevertheless, they share some basic architectural features. These conserved features allow these enzymes to transfer the terminal (gamma) phosphate of either ATP or GTP to the hydroxyl group of serine (Ser), threonine (Thr) and/or tyrosine (Tyr) residues in their target proteins. This common mechanism is achieved by homologous kinase (catalytic) domains, which are about 300 amino acids in length. The catalytic domain is responsible for the binding of the phosphate donor with a divalent cation $\left(\mathrm{Mg}^{+2}\right.$ or $\left.\mathrm{Mn}^{+2}\right)$, and the transfer of the gammaphosphate from ATP/GTP to the target proteins. 
Eukaryotic kinases can be divided into three groups based on their target specificity (Edelman et al., 1987; Yarden and Ullrich, 1988). The first group of kinases is named Ser/Thr protein kinases since they exclusively phosphorylate Ser and/or Thr residues on their targets and this group represents by far the predominant category of protein kinases. The second group only targets tyrosine residues in their substrate proteins and are called tyrosine kinases. The last group includes the uncommon dual specificity kinases, which can phosphorylate Ser/Thr, and Tyr residues, which are referred as the "switch hitters'. In this introduction, I will focus on the eukaryotic Ser/Thr protein kinase CK2.

\section{Protein Kinase CK2:}

As mentioned earlier, the laboratory of Eugene Kennedy discovered casein kinases in the mid-1950s' (Burnett and Kennedy, 1954) including Protein kinase CK2 (a.k.a casein kinase 2). Therefore, CK2 has been under study for more than 50 years. During this time, a significant body of evidence has been gathered about numerous targets and the CK2 mediated cellular events. In this introduction, I focus on various aspects of this kinase which are: a) the composition and structure, b) molecular and genetic studies in model organisms, c) the major targets and biological roles, and d) the regulation.

CK2 is a highly conserved Ser/Thr kinase, which is ubiquitously expressed among eukaryotic organisms (Glover, 1998; Pinna, 1994). Analyses in various model systems, such as yeast and mammalian cell lines have revealed that CK2 is essential for viability (Hanna et al., 1995; Pepperkok et al., 1994). Some of the processes regulated in part by CK2 include cell cycle progression, apoptosis, DNA replication and repair, transcription, translation, and cytoskeletal architecture (reviewed in (Glover, 1998). Over 300 identified substrates that are phosphorylated by CK2 makes this enzyme a global regulator of cell biology (Litchfield, 2003; Meggio and Pinna, 2003). Among the target proteins are enzymes involved in nucleic acid synthesis, e.g., RNA polymerase II and DNA topoisomerase II, 
transcription factors including c-Myc and p53, signal transduction proteins including protein kinase $\mathrm{C}$ and calmodulin, cytoskeletal and structural proteins e.g., beta-tubulin among others (Litchfield, 2003; Meggio and Pinna, 2003). The high number of substrates is, perhaps, reflects the broad distribution of CK2 within the cell. For example, CK2 is found in the cytosol, in the nucleus and is often reflected in the plasma membrane and other cellular compartments such as golgi apparatus and ribosomes (Litchfield, 2003). This distribution appears to reflect its diverse functions with regards to the classes of proteins whose functions are regulated by this enzyme. The minimal consensus sequence for substrate recognition and phosphorylation is (Ser/Thr)-Asp/Glu-x-Asp/Glu where $\mathrm{X}$ can be any amino acid other than Lys/Arg (Kuenzel et al., 1987). Furthermore; CK2 is one of the few kinases that can utilize ATP and GTP with equivalent efficiencies (Tuazon and Traugh, 1990; Tuazon and Traugh, 1991). One enigmatic aspect of CK2 is that its activity appears to be constitutive, and to date; no second messenger has been identified that is required for its regulation. This by no means suggests that CK2 serves simple housekeeping functions, given its roles in processes that are dynamically controlled in vivo.

\section{A) Composition and structure of CK2:}

CK2 is composed of two catalytic alpha subunits (35-44 kDa) and two regulatory beta subunits (24-28 kDa) that associate with each other to generate the $\alpha 2 \beta 2$ heterotetrameric holoenzyme (Glover, 1998; Pinna, 1990; Pinna, 1994). There are two isoforms of the catalytic subunit, $\alpha$ and $\alpha$ ', which are encoded by different genes. Mainly most organisms contain both forms of this subunit with the exception of Drosophila, which contains only the $\alpha$ isoform (Glover et al., 1983). There are three isoforms of the $\beta$ subunit,

$\beta, \beta^{\prime}$ and ssl. Although most organisms contain primarily the $\beta$ isoform, $\beta$ ' is found in Saccharomyces cerevisiae and ssl in Drosophila melanogaster (Bidwai et al., 1999; Karandikar et al., 2003). However, the roles of these isoforms remain unclear. 


\section{A}

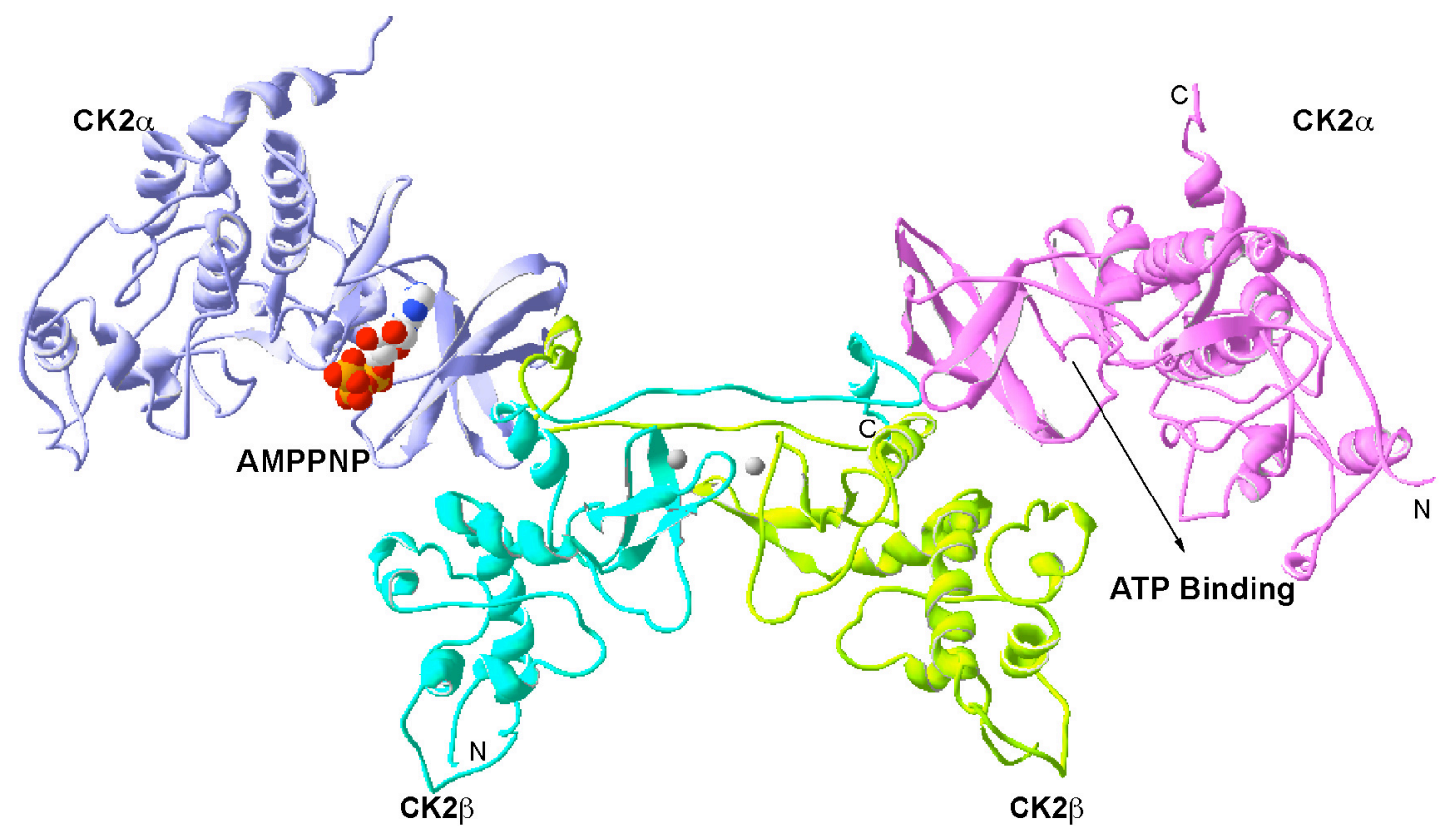

B

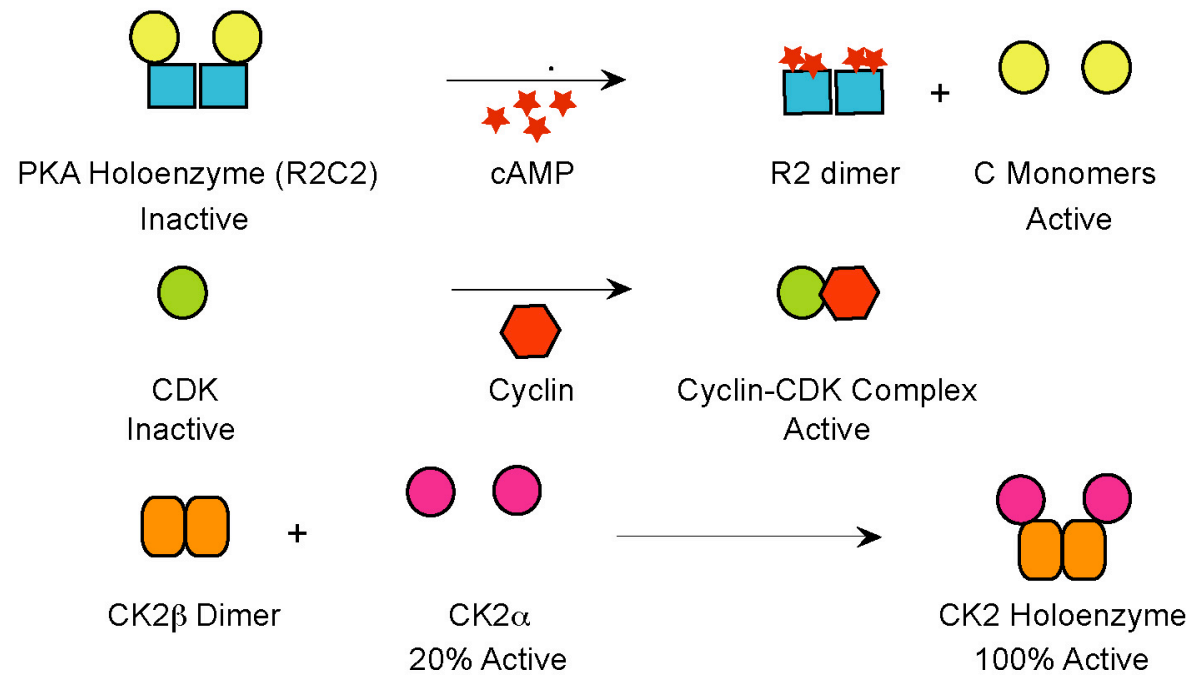

Figure1: Structure of CK2 holoenzyme and activity comparison of CK2 with PKA and

CDK: A) The ribbon diagram represents the tetrameric structure of CK2 holoenzyme $(\alpha 2 \beta 2)$. The two regulatory subunits (shades of green) form the $\beta-\beta$ dimer, which then recruits two $\alpha$ subunits (shades of purple). The active sites are facing outward, which can be visualized by the location of the AMPPNP (unhydrolyzable ATP analogue). The diagram is created with Swiss-PdbViewer (aka DeepView) via using the coordinates of the pdb file 1jwh (Niefind et al., 2001). B) Unlike PKA or CDK, whose activities are tightly regulated at the level of assembly/disassembly of the holoenzyme, CK2 is partially active as a monomer and fully active in a tetrameric conformation. 
Since the tetrameric conformation of CK2 is quite similar to that of CAMP dependent protein kinase (PKA), it was initially thought that CK2 might similarly be regulated by assembly/disassembly of the holoenzyme (Taylor, 1989). PKA is completely inactive in a tetrameric state and association of the regulatory subunits act as on/off switch for the activity (Fig. 1). Another enzyme, which is also regulated via assembly/disassembly is cyclin dependent kinase (CDK), which is completely inactive in a monomeric state and association of the regulatory subunit (cyclin) turns on the enzymatic activity (Draetta, 1990). These, however, turn out not to be the cases for CK2, since both monomeric CK2 $\alpha$ and tetrameric CK2 displays catalytic activity (Fig. 1). Therefore association of the $\beta$ subunit does not act as an on/off switch, but rather acts as a modulator of the enzymatic activity. In a tetrameric form the activity of CK2 increases $\sim 5$-fold towards most target proteins with some exceptions. Calmodulin is one such example, whose phosphorylation is inhibited upon the association of the $\beta$ subunit (Bidwai et al., 1993). In addition, association of CK2 $\beta$ seems to stabilize the holoenzyme against denaturation (Cochet and Chambaz, 1983)

Structurally, CK2 has the general shape of a butterfly with a two-fold symmetry (Fig. 1) (Niefind et al., 2001). The central $\beta$-dimer recruits the two alpha subunits. Specifically, the alpha subunits do not contact each other, and are positioned distal to the central CK2 $\beta$-dimer (Chantalat et al., 1999). The interactions between CK2 $\alpha$ and CK2 $\beta$ occur only at the $\mathrm{N}$-terminal region of the $\alpha$ subunit, which contains a conserved region observed in other kinases (Knighton et al., 1991). This means that no $\alpha$-specific structural element interacts with the $\beta$ subunit, which raises the possibility that other kinases with similar structural elements can also interact with CK2 $\beta$. For instance, kinases such as A-Raf or cMos can form stable complexes with $\mathrm{CK} 2 \beta$, which have been implicated to suggest that CK2 $\beta$ might have other functions (Boldyreff and Issinger, 1997). However, the significance of these interactions with CK2 $\beta$ remain to be confirmed. 


\section{Catalytic subunit ( $\alpha$ and/or $\left.\alpha^{\prime}\right)$ :}

An alignment of CK2 $\alpha$ isoforms (Fig. 2) demonstrates that this subunit is highly conserved throughout evolution (Padmanabha et al., 1990). As mentioned previously, there are two isoforms of this subunit. Although this two forms of the $\alpha$ subunit exhibit $>90 \%$ sequence identity mostly in the $\mathrm{N}$ terminal 330 residues, they are encoded by separate genes (Litchfield, 2001). In contrast to the $\mathrm{N}$ terminal region, the $\mathrm{C}$ terminal region of these isoforms are completely unrelated. This may suggest unique cellular functions for each isoform. Based on genetic studies conducted in S. cerevisiae, the two isoforms can compensate for each other to maintain cellular viability (Hanna et al., 1995; Pepperkok et al., 1994). However, each isoform is also found to have separate functions. The $\alpha$ isoform encoded by CKA1 gene is required to establish cell polarity in yeast (Rethinaswamy et al., 1998). In contrast, CK2 $\alpha^{\prime}$ encoded by CKA2 gene is involved in cell cycle progression (Hanna et al., 1995; Pepperkok et al., 1994). Similarly, isoform specific functions are also observed in mammals based on the evidence that they have different subcellular localization at different stages of the cell cycle (Litchfield, 2001). In addition to the cellular position, the $\alpha$ and $\alpha^{\prime}$ isoforms are differentially phosphorylated during cell division (Litchfield, 2001). The unique $\mathrm{C}$-terminal region of the $\alpha$ isoform contains multiple phosphorylation sites and also a "PXXP" motif, a SH3 domain interaction motif (Litchfield, 2001). Additional support for functional diversity is derived from the evidence that $\alpha$, but not $\alpha^{\prime}$, can interact with the phosphatase PP2A (Heriche et al., 1997). Another protein that can interact with $\alpha$ but not with $\alpha^{\prime}$, is CK2 interacting protein 1 (CKIP-1) (Bosc et al., 2000; Litchfield, 2001). Both of these $\alpha$ specific interacting partners will be discussed in the regulation of CK2 section of this thesis. However, the precise functions of each isoform remain to be discovered in mammals. 




Figure 2: Alignment of CK2 $\alpha$ subunits. The sequence alignments of CK2 $\alpha$ among different species were done using the multiple sequence alignment software (ClustalW). The various alleles of CK2 are indicated and listed. Dm-Drosophila melanogaster, XI-Xenopus laevis, Gg-Gallus gallus, HsHomo sapiens. 
The structure of CK2 $\alpha$ shares some fundamental architectural elements with other eukaryotic protein kinases: These include $\beta$-sheet rich $\mathrm{N}$-terminal domain, the $\alpha$-helical $\mathrm{C}$ terminal domain and the active site that lies in the interfacial region (Fig. 3). The N-terminal extension contains conserved sequence motifs "SEARVY" and "EYWDYE", which act to stabilize the catalytic hinge region upon substrate binding (Kudlicki et al., 1984). The activation segment $\left(\mathrm{Asp}^{175}\right.$ to $\left.\mathrm{Glu}^{201}\right)$ is attached to the $\mathrm{N}$-terminal segment of the alpha subunit without interacting with the beta subunits (Niefind et al., 2001). This negates the possibility that CK2 $\beta$ binding changes the conformation of this segment as in CDK. For example, the CDK2 activation segment acts like an on/off switch upon cyclin A binding (Solomon et al., 1990). However, in the case of CK2, the conformation of the activation segment remains unchanged in its monomeric/tetrameric status (Niefind et al., 2001).

\section{Regulatory subunit ( $\beta$ and/or $\left.\beta^{\prime}\right)$ :}

The CK2 $\beta$ subunit does not share sequence similarity with any known protein, however, it is highly conserved with its homologs in other species (Fig. 4). For example, human and Xenopus CK2 $\beta$ 's differ by only a single residue. The structure of the beta subunit can be divided into four portions: the $\alpha$-helical $\mathrm{N}$ terminal domain, the acidic region, the Zinc finger (juxta-dimer interface) region and the C-terminal tail (Fig. 3). First, the $\mathrm{N}$ terminal domain contains two autophosphorylation sites at $\mathrm{Ser}^{2}$ and $\mathrm{Ser}^{3}$, which are highly conserved between species (Fig. 5) (Meggio et al., 1983). Autophosphorylation is proposed to influence binding of other proteins, such as Nucleolar phosphoprotein Nopp140, and ribosomal protein L41 (Meier and Blobel, 1992). However, the significance of this region or its phosphorylation still remain unclear. This $\mathrm{N}$ terminal domain also contains a putative 'destruction box', which is located close to the autophosphorylation sites (Jauch et al., 2002). This destruction box is a conserved sequence motif of nine amino acids




A

$\operatorname{CK} 2 \alpha$

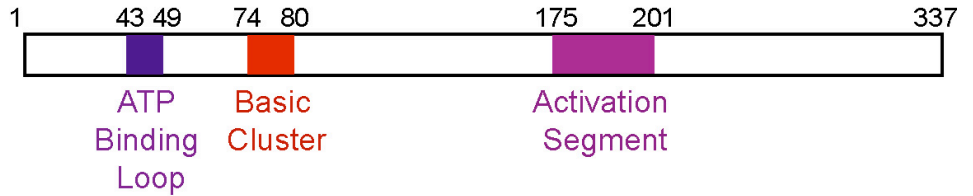

CK2 $\beta$



B $\operatorname{CK} 2 \alpha$

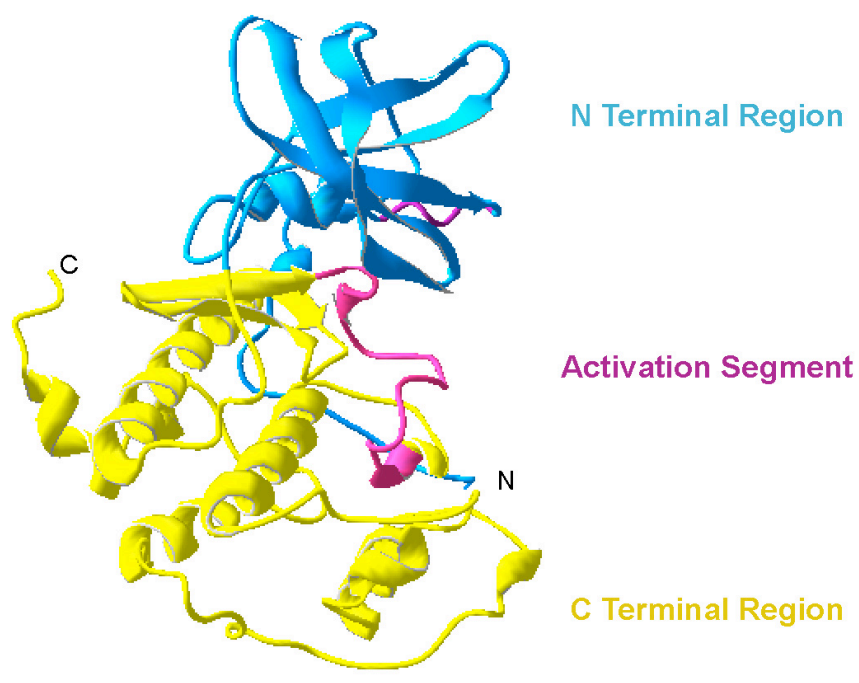

C $\quad$ CK2 $\beta$

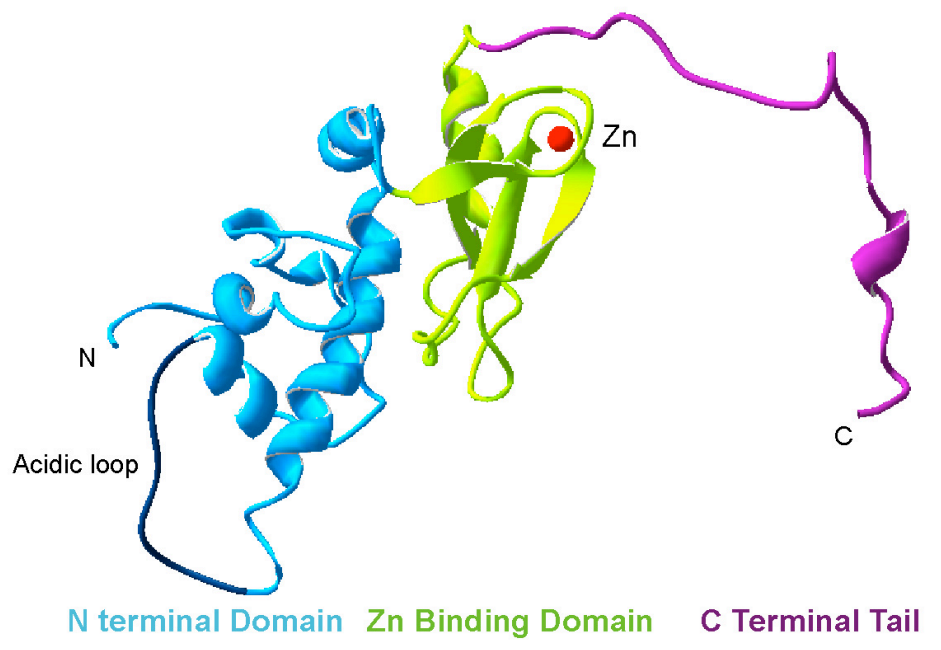

Figure 3: Functional domains of CK2 $\alpha$ and CK2 $\beta$ : A) Schematic of various functional domains of CK2 $\alpha$ and CK2 $\beta$. B) Ribbon diagram of CK2 $\alpha$ showing different functional domains, the $\mathrm{N}$ terminal region (blue), the activation segment (pink) and the $C$ terminal region (yellow). C) Ribbon diagram of CK2 $\beta$ showing different functional domains, the $\mathrm{N}$ terminal domain (blue), the Zinc finger domain (green) and the $\mathrm{C}$ terminal tail (puple). (pdb file $1 \mathrm{jwh}$ ) 
(Allende and Allende, 1995). Second, the acidic loop region, from $\mathrm{Asp}^{55}$ to $\mathrm{Asp}^{64}$, conserved and thus proposed to be critical for modulation of CK2. However, loss of this internal acidic region does not change the assembly or activation of CK2 $\alpha$ but affects specificity that suggests a putative regulatory role for this region. Third, the juxta-dimer interface region or the Zinc finger domain is required for $\beta$ dimerization, an interaction that is vital for holoenzyme assembly. There are four conserved cysteine residues that mediate the dimerization and recruitment of the $\alpha$ subunit, Cys ${ }^{109}$, Cys ${ }^{114}$, Cys $^{137}$ and Cys ${ }^{140}$ (Fig. 5). This also means that the dimerization is required prior to holoenzyme formation. Lastly, the C terminal tail contains a second phosphorylation site at $\operatorname{Ser}^{209}$ residue, which is catalyzed by $\mathrm{p} 34^{\mathrm{cdc} 2}$ in a cell cycle dependent manner (Bosc et al., 1995; Litchfield, 2003). This is not vital for in vivo function but appears to render the enzyme less efficient for cell cycle progression (Litchfield, 2003).

\section{B) Molecular and genetic studies in model organisms:}

Over the years, there has been an enormous amount of information discovered about CK2 and its functions. Subcloning the cDNA of CK2 made it possible to study the specific roles of this enzyme in vivo by using various model systems. The initial studies employed yeast S. cerevisiae and revealed various roles of CK2 during cell cycle progression, which positioned CK2 in the 'pantheon' of important enzymes (Glover et al., 1994). Other model organisms have also been used over the years; however, for the relevance to my studies, I will only discuss two of them, yeast and Drosophila.

\section{Alleles of CK2:}

To date two alleles of CK2 $\alpha$, timekeeper (Tik) and its partial revertant TikR were identified in Drosophila (Fig. 6) (Lin et al., 2002). Tik was identified as a dominant modifier of the circadian clock during a screen for clock defects. Molecular analysis demonstrated 


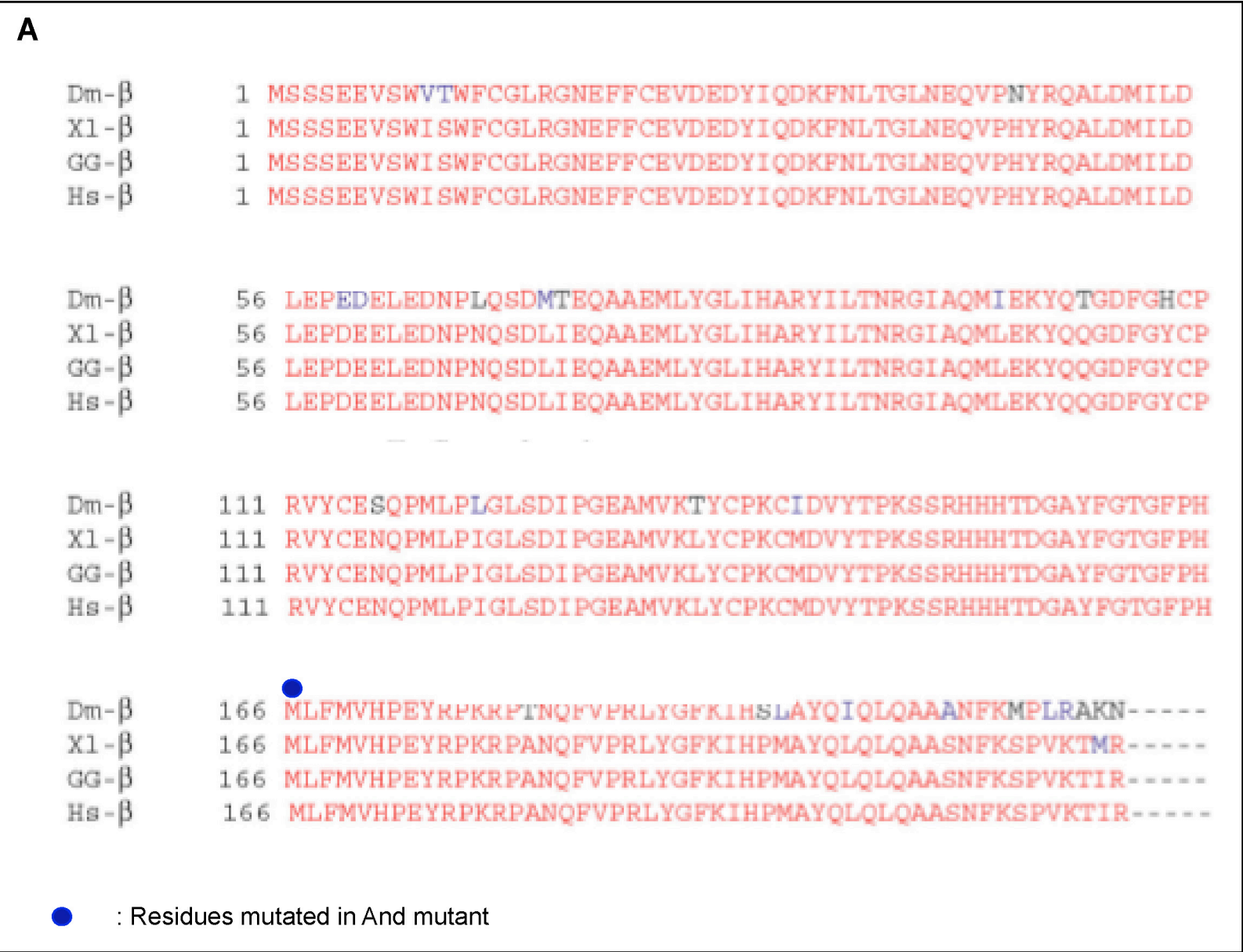

Figure 4: Alignment of CKb subunits. The sequence alignments of CK2 $\beta$ subunit amongst various species were done using the multiple sequence alignment software (ClustalW). And allele in Drosophila is indicated.. Dm-Drosophila melanogaster, XI-Xenopus laevis, Gg-Gallus gallus, HsHomo sapiens. 
that Tik harbors two substitutions, $\mathrm{M}^{161} \mathrm{~K}$ and $\mathrm{E}^{165} \mathrm{D}$. The former residue is invariant amongst all isoforms of CK2 $\alpha$ that have been characterized to date. However, the latter residue is conserved only in metazoan organisms. In the crystal structure of human CK2 $\alpha$, $\mathrm{Met}^{161}$ is located within the ATP binding pocket, which is somewhat hydrophobic (Niefind et al., 2001). The substitution of this non-charged residue with a positively charged Lys is predicted to interfere with ATP binding and thus negatively impact phosphorylation. Consistent with this prediction, recombinant bacterially expressed Tik displays no discernible catalytic activity (Lin et al., 2002). As a result, Tik homozygotes are larval 1 lethal (Lin et al., 2002). While it has not been formally demonstrated, the second substitution in Tik (Glu ${ }^{165}$ to Asp) is thought to not contribute to the loss of function (activity) behavior. This possibility is likely given that this residue is not conserved in all CK2 $\alpha$ isoforms and its position in a hinge region facing away from the active site would argue against a role in mediating intramolecular contacts or interactions with CK2 $2 \beta$. The second allele of CK2 $\alpha$ is TikR, a partial revertant that displays attenuated defects of the circadian clock when compared with Tik. It was found that TikR harbors the two original mutations found in Tik, and in addition deletes seven amino acids (234-240) and substitutes an additional $\operatorname{Arg}^{242}$ Glu (Lin et al., 2002). Given the presence of the $M^{161} \mathrm{~K}$ substitution, TikR is predicted to display no catalytic activity, and is thus lethal like Tik when homozygous. Thus, both Tik and TikR abrogate CK2 activity. The question that arises from these findings is why then does TikR behave as a 'revertant'. One possibility is that the internal deletion in TikR compromises proper folding and thus its overall structure is deformed (Lin et al., 2002). As a result, the TikR protein might be unable to make a complex with endogenous CK2 $\beta$ to generate the tetrameric holoenzyme (Lin et al., 2002). This, however, is unlikely to be the case for Tik, which might be able to make a complex and poison the holoenzyme, thus acts as a dominant-negative. 


\section{A CK2 $\beta$}

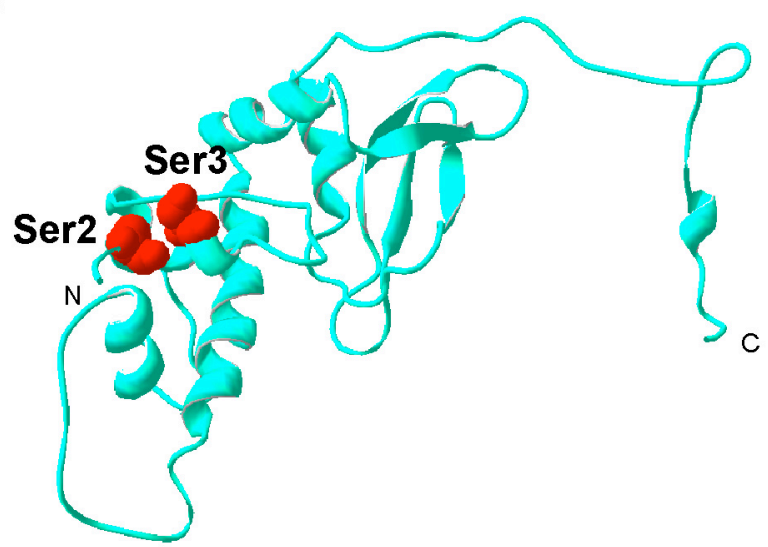

B $\mathrm{CK} 2 \beta$

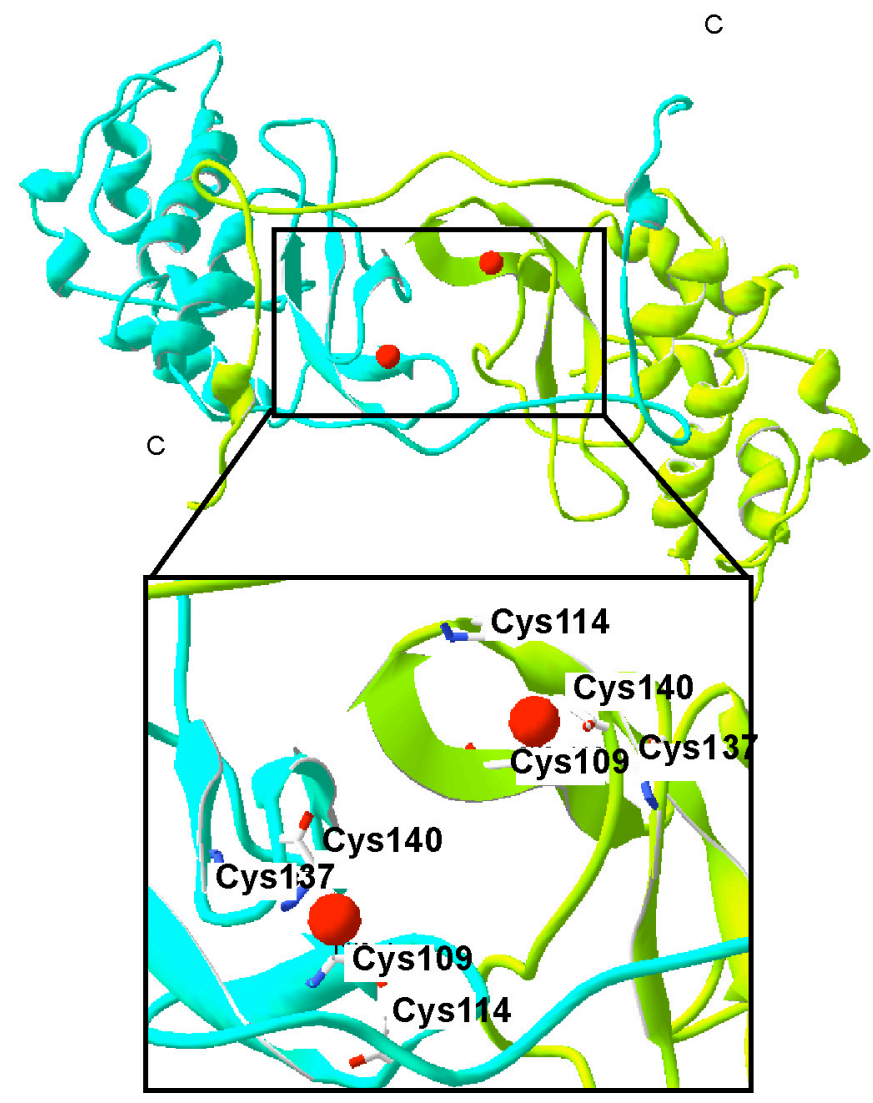

Figure 5: Structure of CK2 $\beta$ autophosphorylation and Zinc binding domains: A) Two autophosphorylation sites on the immediate $\mathrm{N}$ terminal region of CK2 $\beta$, Ser2 and Ser3 (red), are shown in the ribbon diagram. B) The ribbon diagram representing the zinc finger domain found in CK2 $\beta$ mediates $\beta$-dimerization ( $\mathrm{pdb}$ file $1 \mathrm{jwh}$ ). There are four essential (and conserved) Cys residues in each subunit which are positioned to surround the $\mathrm{Zn}$ ion (red). 
There is only a single allele for Drosophila CK2 $\beta$, Andante $(A n d)$, which is also discovered as a result of an altered clock behavior (Fig. 6) (Rasmussen et al., 2005). And contains a single amino acid substitution, $\mathrm{M}^{161} \mathrm{I}$, which is located at the $\alpha / \beta$ interface region (Rasmussen et al., 2005). This substitution is proposed to disrupt the interaction between $\alpha$ and $\beta$ and elicits lengthened clock behavior (Rasmussen et al., 2005). However, this proposition is controversial since some experimental data of human CK2 $\beta$ with the $\mathrm{M}^{161}$ I mutation does not support this prediction (Rasmussen et al., 2005). For the interest of this thesis, And will not be discussed any further.

\section{S. cerevisiae:}

The budding yeast, S. cerevisiae, is one of the first organisms, in which CK2 functions were studied via the genetic routes. The initial findings from mutagenic analysis suggested a role for the yeast CK2 $\alpha$ gene $(\mathrm{yCK} 2 \alpha)$ in cell viability, as deletion of both of the catalytic subunits was lethal (Padmanabha et al., 1990; Rasmussen et al., 2005). These cells displayed "shmoo" phenotypes (pseudomycelia), which are a hallmark of cell cycle defects in this organism (MacKay, 1974). It was shown that cDNAs encoding Drosophila CK2 (dCK2) could rescue the lethality, illustrating high functional conservation of CK2 over 500 million years of evolution (Bidwai et al., 1992). This presented a great opportunity for scientists to study the functions of CK2 from different organisms by testing for yeast complementation. Furthermore, yeast also enables the isolation of temperature sensitive alleles of CK2 $\alpha$, which encode for functional proteins at permissive temperatures that are non-functional at nonpermisive temperatures. In contrast to catalytic subunits, deletion of the genes $C K B 1$ and $C K B 2$ encoding the regulatory subunits has no effect on viability, but renders cells to be salt sensitive (Bidwai et al., 1995).

\section{D. melanogaster:}


Starting from early twentieth century, Drosophila has been employed as a model organism. Thomas Hunt Morgan was the first to employ Drosophila as a model organism to study genetics. He was awarded with a Nobel Prize in 1923 for his discoveries on the role of chromosomes in heredity. Although, he and his students employed this model system extensively, Drosophila became a popular model system after the discovery of homeobox genes by Edward Lewis. In addition to Lewis, the studies by Christiane Nusslein-Volhard and Eric Weischaus established a solid place for this model organism in studying genetics and development. The studies of Lewis, Nusslein-Volhard and Weischaus were also awarded with a Nobel Prize in 1995. These findings among others made Drosophila one of the most widely used model organism in developmental biology and genetics today.

Drosophila CK2 is composed of a single isoform of CK2 $\alpha$ (Glover et al., 1983). This is in contrast to other metazoans, which contain two distinct isoforms called $\alpha$ and $\alpha$ ' as mentioned previously. While the holoenzyme purified from Drosophila embryos exhibits the presence of a single beta subunit, the Drosophila genome harbors two additional genes that encode polypeptides that resemble CK2 $\beta$, i.e., $\beta$ ' and SSL (Suppressor of Stellate Like)(Bidwai et al., 1999; Karandikar et al., 2003). The functions and/or relevance of these two alternative CK2 $\beta$-like genes are currently unclear. In Drosophila, CK2 $\alpha$ is localized to the third chromosome, whereas CK2 $\beta$ is X-linked (Bidwai et al., 1999). CK2 plays a crucial role during development of Drosophila. The important CK2 targets include Antennapedia (Antp), Engrailed (En), Cactus and E(spl)M8 (Bourbon et al., 1995; Jaffe et al., 1997; Liu et al., 1997; Packman et al., 1997).

There are multiple ways to study CK2 by using Drosophila as a model organism. The most common methods that were employed to date include forward genetics (phenotype-togenotype) approach or reverse genetics (genotype-to-phenotype). In the case of the forward 


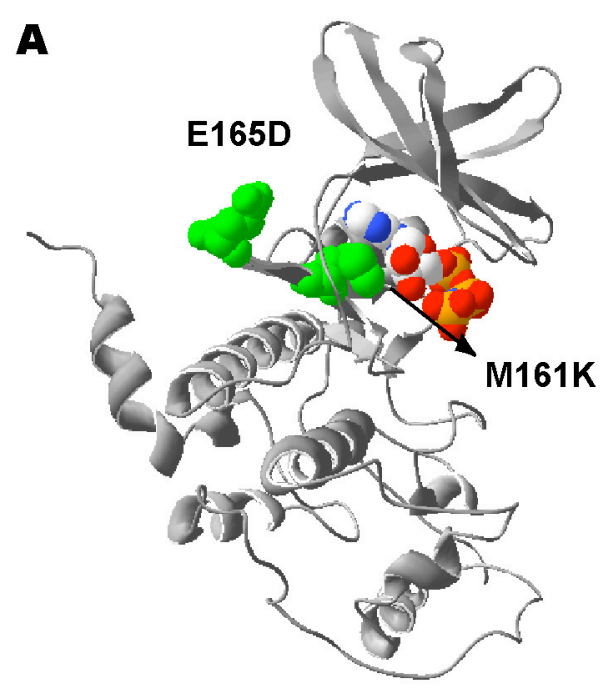

CK2 $\alpha-$ Tik

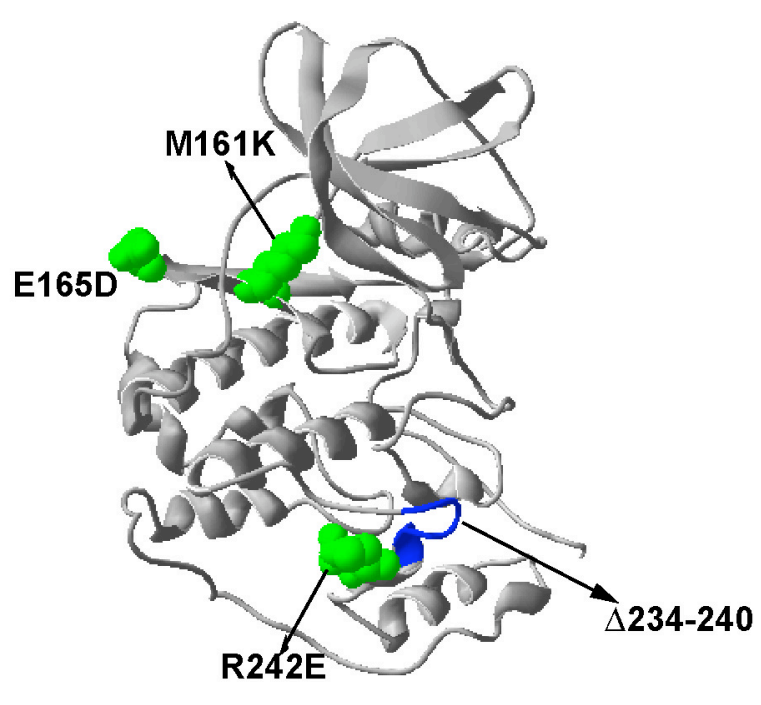

CK2 $\alpha-$ TikR

$\mathbf{B}$

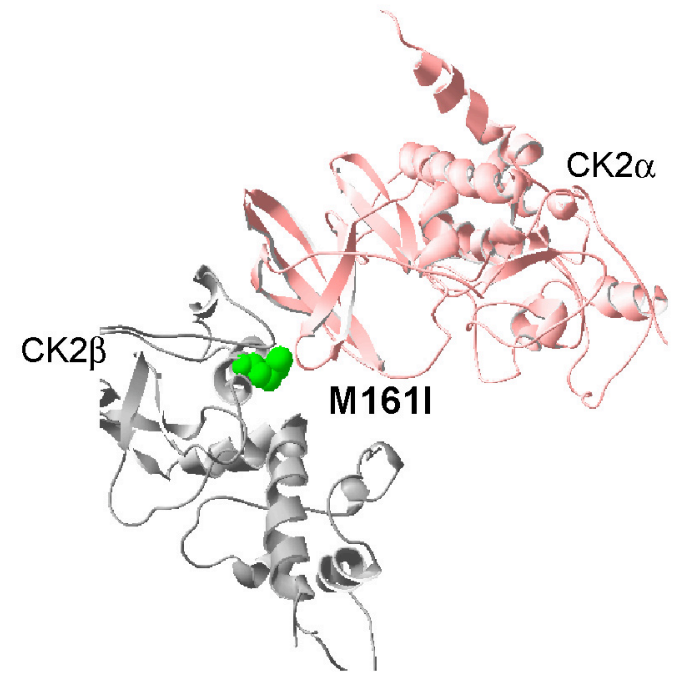

\section{CK2 $\beta$-And}

Figure 6: Structures of CK2 alleles: A) Ribbon diagrams of two alleles of CK2 $\alpha$, Timekeeper (Tik) and its partial revertant (TikR) are depicted. The positions of the amino acid substitutions (green) and/or deletions (blue) are indicated for each allele. B) Ribbon diagram of CK2 $\beta$ allele Andante (And) is shown. The position of the single substitution is indicated in green. 
genetics approach, phenotypes can be detected during mutagenized genomic screens, which are generated via chemical mutagens. The mutated genes can be discovered by mapping the phenotypes in the genome. Mutations in the genes encoding CK2 subunits can be discovered with this method like in the case of Tik, TikR and And. However, it is difficult to obtain viable CK2 mutations from genetic screens due to cell autonomous roles of CK2, which will be discussed in the following section. The alternative method is called 'reverse genetics', which provides scientist to employ knowledge from other model systems such as yeast. A common method is to generate a known point mutation that might alter the activity of CK2 and/or a target of interest and generate transgenic fly stocks by using targeted gene expression. One of the most common methods is Gal4-UAS system, which enables us to study the ectopic expression of a transgene in a tissue or time specific manner (see Methods) (Brand and Perrimon, 1993; Duffy, 2002).

\section{C) Major targets and roles of CK2:}

CK2 is a highly pleiotropic enzyme with more than 300 substrates identified to date (10\% of the cellular 'phosphoproteome') (Meggio and Pinna, 2003; Pinna, 2002). This is a very unusual property for an enzyme, whose complete repertoire of targets still remain to be identified almost fifty years after its initial purification. The feature of CK2 also causes this enzyme to be involved in virtually all aspects of cell biology. Most of the targets that are phosphorylated by CK2 are involved in signaling networks and gene expression and transcription (Pinna, 2002). The major cell autonomous roles that will be discussed in the following pages include cell cycle, cell survival and apoptosis. The major cell specific roles include circadian clock and Notch signaling among others (Fig. 7)

\section{1) Cell autonomous roles of CK2:}

\section{Cell cycle:}

Cell cycle progression is mediated by cyclin-dependent protein kinase (Cdk) family, 


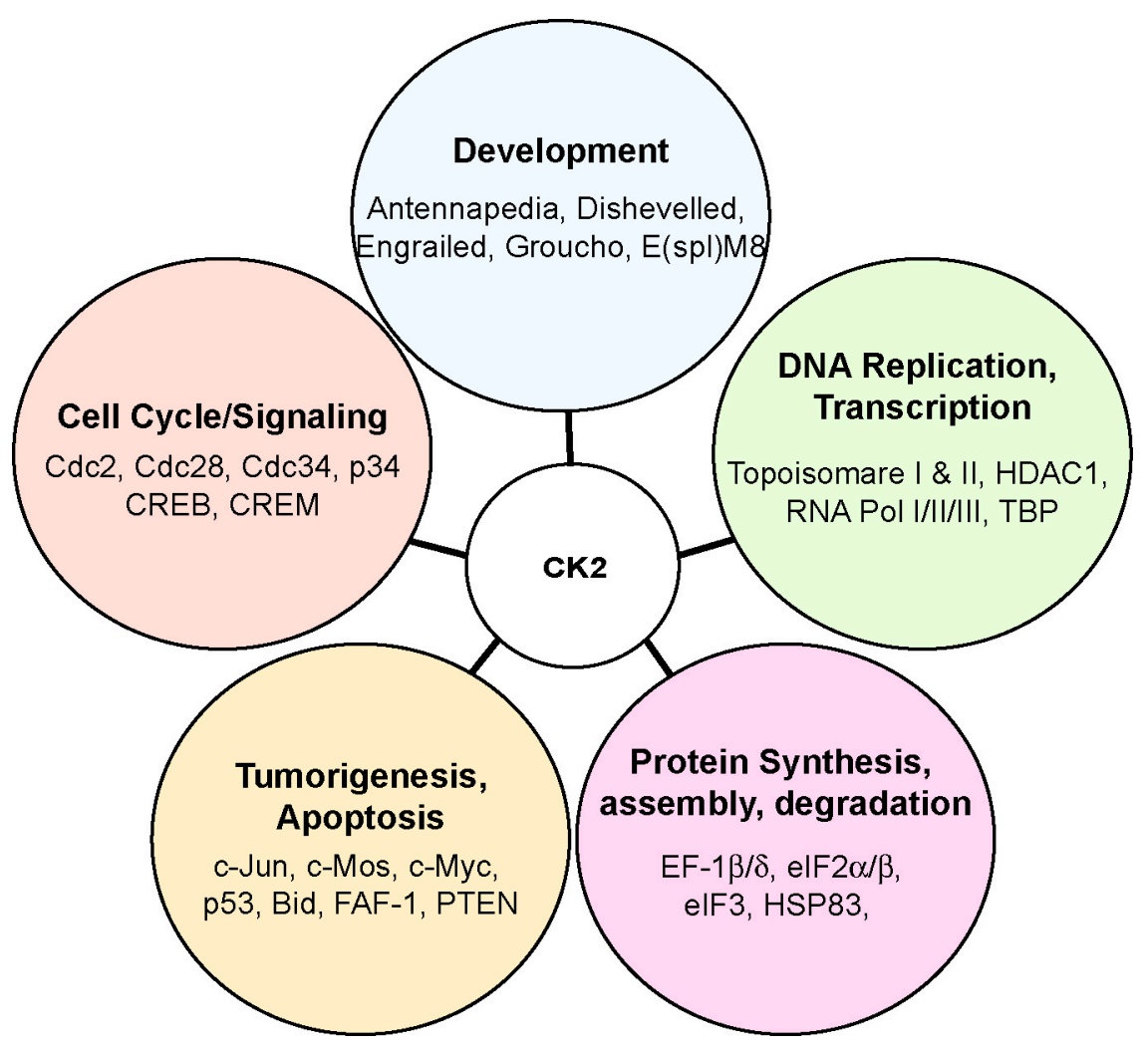

Figure 7: Global roles of CK2: CK2 is a highly pleiotropic enzyme which is expressed ubiquitously. It is involved in a wide variety of cellular processes, some of which are sorted into groups such as development, DNA replication/transcription, protein synthesis/assembly/degradation, tumorigenesis/apoptosis and lastly cell cycle/signaling. This range of involvement in cellular metabolism results in a wide range of target proteins. Some of the significant targets are represented in the figure. These targets have been either identified by direct phosphorylation in vitro/in vivo, or predicted based on the presence of the consensus sequence. 
which is regulated positively by Cdk-activating kinase (CAK) and by association with cyclins and negatively by Cdk inhibitors (CDIs) (Kaldis, 1999). The active form of CDKs can phosphorylate downstream targets, which can regulate the entry into the next phase of the cell cycle (Kaldis, 1999). CK2 is involved in cell cycle progression since the activity of the enzyme rapidly increases during cell division both in normal and cancerous cells, and oscillates in coordination with the G1/S and G2/M phases of the cell cycle (Glover, 1998). In addition, a number of proteins involved in the cell cycle are phosphorylated by CK2 such as cyclin-dependent protein kinase p34 ${ }^{\mathrm{cdc} 2}$ and cdc34, which play an essential role for cell division (Russo et al., 1992). Genetic analyses in yeast have provided insights into the function of CK2 during cell cycle progression. Disruption of CK2 in temperature sensitive yeast strains leads to cell cycle arrest either at the G1/S or G2/M transitions (Russo et al., 1992).

\section{Cell survival and apoptosis:}

Although CK2 does not play a single major role in the ladder of cellular functions, combinatorial loss of all CK2 regulated events results in lethality (Padmanabha et al., 1990). For instance, among the number of CK2 targets, RNA polymerase III (Pol III) machinery plays an essential role for cell survival (Ghavidel and Schultz, 2001). The core component of RNA Pol III, TATA-binding protein (TBP), subunit of TFIIIB, is phosphorylated by CK2, which in turn changes the activity of this complex (Ghavidel and Schultz, 2001). Thus, compromising CK2 activity causes a decline in overall tRNA and 5SrRNA synthesis, which is mediated by RNA Poll III complex (Ghavidel and Schultz, 2001). In addition, CK2 is involved in caspase-mediated apoptosis via phosphorylation of key elements such asHS1, Max, and Bid (Litchfield, 2003; Olsen et al., 2006; Ruzzene et al., 2002). Phosphorylation by CK2 downregulates the caspase-dependent degradation of these pro-apoptotic proteins, suggesting that, CK2 antagonizes apoptosis. All of these studies support the idea that CK2 
plays a key role for cell survival. Over the years, the evidence suggests that CK2 is involved in cancer (Litchfield, 2003). There are number of targets, such as, p53 (tumor suppressor) and c-Myc, c-Myb and c-Jun (protooncogenes), which are all negatively regulated by CK2 (Filhol et al., 1992; Henriksson, 1996; Oelgeschlager, 1995). In addition, the level of CK2 increases drastically in human tumors (Litchfield, 2003).

\section{2) Cell specific/developmental roles:}

\section{Wnt signaling:}

Wnt signaling is an important signaling pathway during development and cancer (reviewed in (Cadigan, 1997)). The name comes from the combination of Drosophila wingless (wg) gene (mutants without wings) and human homologoue of wg, int-1 gene. Binding of the Wnt proteins to the receptors of the Frizzled (Frz) family starts the canonical Wnt pathway (Cadigan, 1997). Receptor activation elicits the activation of Dishevelled (Dsh) family proteins, which in turn inactivate axin/GSK-3/APC complex ( $\beta$ F-catenin destruction complex) (Polakis, 2000). Therefore, ultimately this results in stabilized $\beta$-catenin levels in the cytoplasm, which translocate into the nucleus. There are multiple protein kinases and protein phosphatases that regulate the Wnt pathway. The reversible phosphorylation plays a crucial role during Dsh binding to the $\beta$-catenin destruction complex (Cadigan, 1997). One of the kinases that are involved in this pathway is CK2, which can interact with nuclear $\beta$ catenin and Dsh (Gao and Wang, 2006). It has been shown that CK2 positively affects the translocation of $\beta$-catenin and is required for the proper actions of Wnt-enhancer complexes (Gao and Wang, 2006).

\section{Circadian clock:}

Circadian clocks are internal timekeepers that provide the daily rhythmicity, which is orchestrated via three components: input pathways, central oscillator and output pathways. 
The input pathways are the events in which environmental cues, such as temperature and light, are transduced to the oscillator (reviewed in (Allada, 2003; Berger, 2004). The oscillator is the core of the clock that provides the rhythmicity via molecular feedback loops. Lastly, the output pathways are the biological processes that are regulated via the oscillator.

Drosophila is the first organism where the core components of circadian mechanism were discovered. There are two regulatory feedback loops that generate the rhythm. In the first loop, CLOCK (CLK) and CYCLE (CYC) proteins form a dimer and bind to the promoters of period (per) and timeless (tim) genes (Allada, 2003). This binding then leads to transcription of PER and TIM proteins, which function in the second loop. In the second loop, PER and TIM proteins form a dimer and inhibit their own transcription by removing CLK and CYC. It takes about 24 hours to complete these two loops, which are synchronized to light and dark schedule.

The feedback loops are regulated at different levels such as transcriptional, posttranscriptional and posttranslational that predominantly involves protein-protein interactions and programmed protein turnover (Allada, 2003). Phosphorylation as a means of posttranslational modification has a key role during this process for the maintenance and length of the circadian period. CK2 is the only common kinase that plays an important role in different circadian systems of fungi, plants, and animals, and thus represents an evolutionary link among different species (Akten et al., 2003). In Drosophila, CK2 is expressed in the circadian pacemaker neurons and plays an important role during posttranslational modification of PER (Lin et al., 2002). The mutant flies for CK2 $\alpha$ (TimekeeperTik) exhibit an abnormally long circadian periods and delayed nuclear entry of PER (Lin et al., 2002). Mutant flies for CK2 $\beta$ (Andante-And), also display lengthened clock and accumulation of PER and TIM proteins in the cytoplasm (Rasmussen et al., 2005). Given 
that Tik and And flies do not exhibit other developmental defects, it is plausible to suggest that CK2 plays a key function, which seems to be rate limiting in the molecular clock.

\section{Notch signaling:}

Notch $(\mathrm{N})$ signaling is one of the most important signaling pathways involved in cell fate specification during various developmental processes such as neurogenesis, oogenesis and myogenesis both in invertebrates and higher organisms (reviewed in (ArtavanisTsakonas et al., 1995; Artavanis-Tsakonas et al., 1999; Lai, 2004)). The N signaling pathway has been exceptionally well studied in Drosophila neurogenesis, i.e. eye and bristle development. The main components of $\mathrm{N}$ signaling in this organism are: the membrane receptor Notch $(N)$ (, its ligand Delta $(D I)$, the transcription factor Suppressor of Hairless $(S u(H)), A S C$ proteins and ato, and the members of the Enhancer of Split $(E(s p /))$ complex, which are the ultimate targets of activated $\mathrm{N}$. The $E(s p /)$ locus encodes for seven basic helix loop helix (bHLH) proteins and a non bHLH protein Groucho (Gro) (Delidakis and ArtavanisTsakonas, 1991).

Emerging evidence now suggests that CK2 plays an important role in mediating the effects of the $\mathrm{N}$ signaling pathway during eye and bristle development. Studies from our laboratory suggest that the $\mathrm{E}(\mathrm{spl})$ repressors are regulated by CK2 (Bose et al., 2006; Karandikar et al., 2004). It was found that substitution of the CK2 phospho-acceptor of E(spl)M8 with a 'phospho-mimetic' Asp generates a dominant allele that accentuates bristle development and blocks eye development in a manner similar to hyperactivity of $\mathrm{N}$ signaling (Karandikar et al., 2004). These results have now been corroborated by the finding that compromising CK2 levels/activity by RNAi or dominant negative (UAS-Tik) constructs compromises lateral inhibition (Bose et al., 2006). 


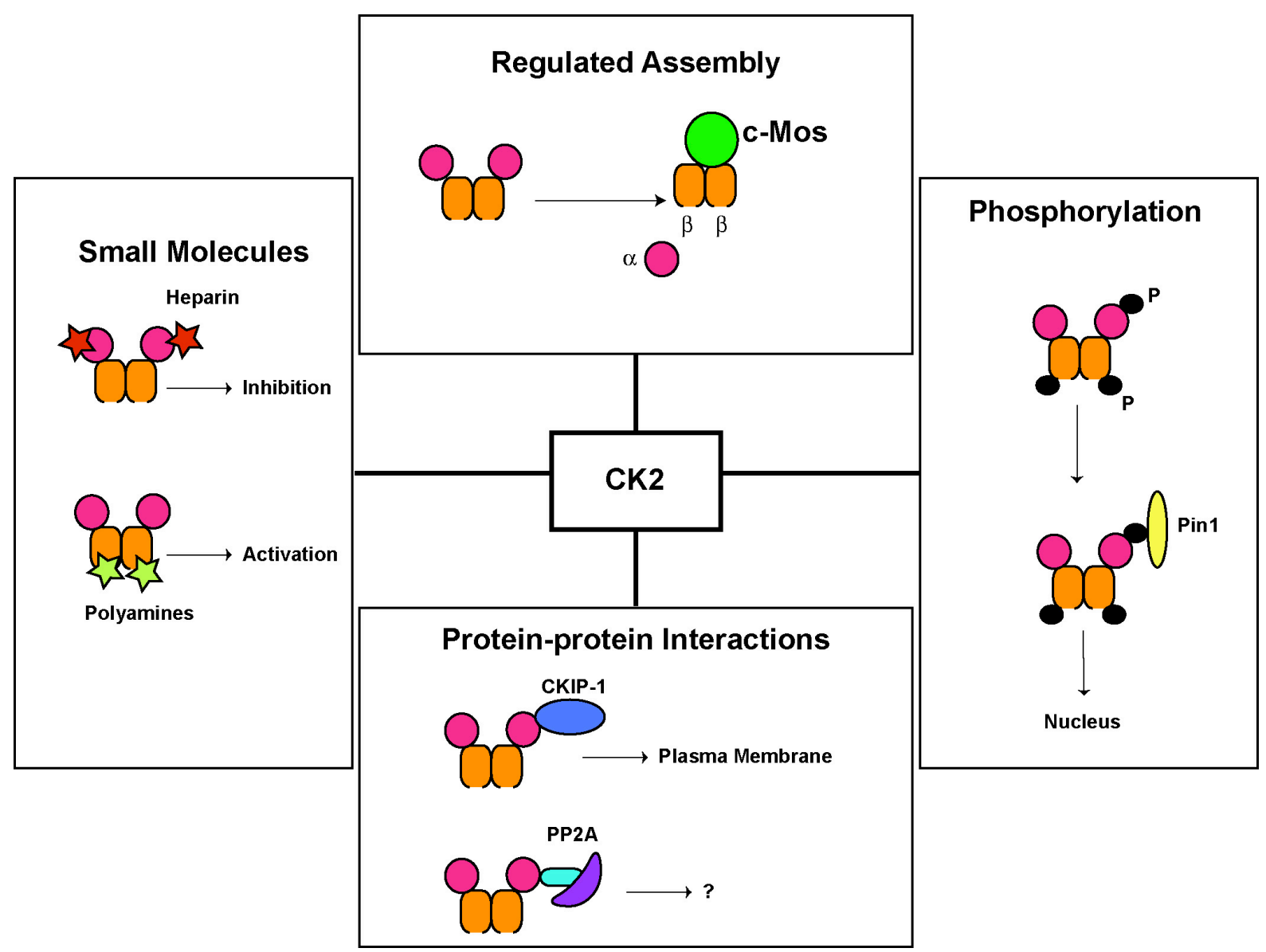

Figure 8: Regulation of CK2: The possible means to regulate CK2 activity, which can be categorized into assembly/disassembly of the holoenzyme, post-translational modifications (phosphorylation), small molecules and protein-protein interactions. 
The mutants of $N$ exhibit severe developmental defects in Drosophila, one of which is the recessive split allele identified in 1987 by the laboratory of and Spyros Artavanis-Tsakonas. A single amino acid substitution $1 \mathrm{e}^{578}$ to $\mathrm{Thr}$ in the extracellular domain of $\mathrm{N}$ elicits highly reduced and rough eyes as well as split and missing bristles (Li et al., 2003). In these mutants the cells that are supposed to gain a neural fate become sensitive to the inhibitory actions of $\mathrm{N}$, thus, become non-neural cells ( $\mathrm{Li}$ et al., 2003). There are multiple paralogs of the Drosophila $\mathrm{N}$ and its components in mammalian system. Therefore, alterations in $\mathrm{N}$ function are found to underlie in number of human diseases including leukemia, CADASIL (hereditary stroke disorder) and chronic liver diseases (Tournier-Lasserve, 1998). Therefore, it is vital for us to study the details of this pathway. I will talk, in detail, about $\mathrm{N}$ signaling during eye and bristle neurogenesis later in this chapter.

\section{D) Regulation of CK2:}

Regulation of CK2 has been disputed over the years due to the existence of multiple, sometimes contradictory, mechanisms. These mechanisms can be divided as: the assembly/disassembly of the holoenzyme, covalent modifications (phosphorylation and ubiquitination), small molecules (polyamines), and protein-protein interactions (CKIP and PP2A) (Fig. 8).

\section{1) Assembly/disassembly of the holoenzyme:}

Assembly/disassembly of a holoenzyme is an important mechanism for some enzymes, such as CDKs. Recruitment of regulatory subunits (cyclins) are essential for the activity of CDKs, thus, assembly of the holoenzyme is the sole mechanism to regulate the activity (Draetta, 1990). In the case of CK2, however, the recruitment of the regulatory subunit increases the activity of the enzyme for most of the target proteins with some exceptions. As mentioned before, Calmodulin has a lower affinity towards binding to the 
holoenzyme (Bidwai et al., 1993). Therefore, assembly/disassembly of the CK2 holoenzyme cannot be the sole mechanism to regulate the enzymatic activity but seems to modulate the enzyme. In parallel to this, the observation that CK2 $\beta$ can form stable complexes with proteins like c-Mos or a-Raf, raises the possibility that CK2 assembly might actually be a regulatory mechanism (Litchfield, 2003).

\section{2) Posttranslational modifications:}

There are multiple posttranslational modifications that have been suggested to regulate CK2, such as phosphorylation and ubiquitination (Litchfield, 2003). First, phosphorylation of the activation loop is a common mechanism to switch on the catalytic activity of numerous protein kinases. For instance, MAP kinases are inactive in the nonphosphorylated form but are switched on by phosphorylation (Goldsmith, 1998). Unlike these enzymes, however, CK2 displays a robust level of activity independent of its phosphorylation status (Litchfield, 2003). There are multiple phosphorylation sites on CK2 $\alpha$ and CK2 $\beta$. The 4 phosphorylation sites on CK2 $\alpha$ (but not $\alpha^{\prime}$ ) are located in the unique C terminal domain, which might influence the binding of other proteins to this region and target specificity (Litchfield, 2001). Furthermore, the autophosphorylation sites on CK2 $\beta$ are phosphorylated via CK2 $\alpha$ at $\operatorname{Ser}^{2}$ and Ser3 in a cell cycle dependent manner (Bidwai et al., 1995). Mutational analysis of these sites did not elicit loss of function but rendered the enzyme less efficient in vitro (Zhang et al., 2002). Autophosphorylation event has been suggested to increase the stability of the holoenzyme against ubiquitination. In the absence

of CK2 $\beta$, CK2 $\alpha$ has been found to undergo phosphorylation at $\operatorname{Tyr}^{182}$, which is located within the activation loop (Donella-Deana, 2001). However, the implications of this event still remain to be resolved. 
A



CK2 $\alpha^{\prime} \stackrel{1}{\longrightarrow}^{350}$



B



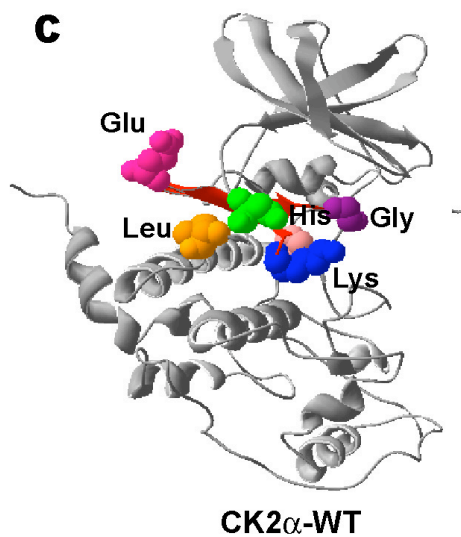

D

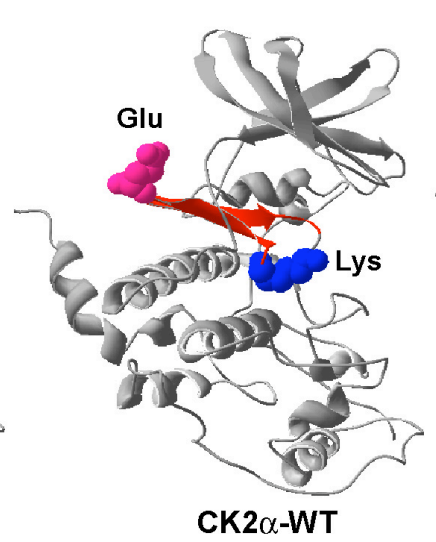

CK2 $\alpha-W T$

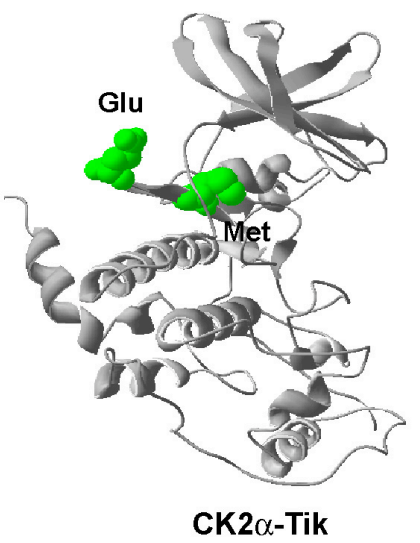

CK2 $\alpha-$ Tik



Figure 9: CK2 interacting protein-1 (CKIP-1) and HIKE motif. A) The schematic

representing unique $\mathrm{C}$-terminal domains of $\mathrm{CK} 2 \alpha$ and $\mathrm{CK} 2 \alpha$, which can differentially interact with CKIP-1 through its PH domain. B) CK2 contains a 'HIKE' domain that is found in strong PH domain interacting proteins (alignment from (Ciccarelli FD, 2000)). C) The five HIKE residues (Asp, Leu, His, Lys, Glu and Gly). Two of these are thought to be critical, which are depicted separately (Lys (blue) and Glu (pink)). The aminoacid substitutions found in Tik are also shown since Glu residue is common between HIKE domain and Tik. D) The signature structural element in HIKE, $\beta$-stand-loop- $\beta$ strand, is shown in red. The locations of Tik mutations are shown are shown on that strand. 


\section{3) Small molecules:}

Second messenger dependent kinases are regulated via small molecules (cAMP, $\mathrm{Ca}^{++}$or lipids). CK2 is not a member of this group of enzymes, which makes it harder to elucidate the regulatory mechanisms. Although the second messengers like cAMP are not involved with CK2, other small molecules seem to interact with CK2. In vitro studies indicate that CK2 is inhibited by negatively charged molecules like heparin and activated by positively charged molecules like polyamines (Hathaway et al., 1980; Hathaway and Traugh, 1984). In vivo studies in mice also supported the idea that polyamines activate CK2 activity via interacting with the CK2 $\beta$ subunit. However, the in vivo significance and precise method of these types of interactions between small molecules and CK2 needs to be clarified.

\section{4) Protein-protein interactions:}

Protein-protein interactions are another means to regulate the activities of protein kinases. There are number of kinases that can be regulated via interacting with other proteins, which can directly or indirectly influence the activity. For instance, the interaction between CDK2 and the inhibitory protein p21 abrogates catalytic activity of the kinase, which is precisely controlled during cell cycle progression through the $\mathrm{S}$ phase (Harper et al., 1993). Protein-protein interactions might also alter target specificity or localization of a kinase. An excellent example is the A-kinase anchor protein 5 (AKAP5), which alters localization of PKA by binding to its regulatory subunits (Coghlan et al., 1995).

CK2 has multiple interaction partners, which might act directly or indirectly by changing the activity, specificity, or localization. Interaction partners like Hsp90 or Cdc37 influence the catalytic activity directly (Bandhakavi et al., 2002; Miyata and Yahara, 1992). For instance, Hsp90 binds and protects CK2 from self-aggregation and enhances its catalytic activity. In the case of Pin1 and FACT complex, however, the interaction changes 
the target specificity (Litchfield, 2003). Thirdly, proteins like CKIP-1, Tubulin and FAF-1 change the localization of CK2 within the cell (Litchfield, 2003). There are other interacting partners of CK2, whose impacts have not yet resolved, such as phosphatase PP2A (Heriche et al., 1997). Therefore, it is important to study the partners of CK2 to shed light on how it is regulated in vivo. The remaining part of the discussion will focus on two of these interacting partners, which are related to this work.

\section{CK2 interacting protein 1(CKIP-1):}

As mentioned earlier, the C-terminal domains of CK2 $\alpha$ and CK2 $\alpha$ ' are unique to each isoform (Fig. 9). This dissimilarity has been suggested to be responsible for the functional specialization of CK2 isoforms by specific recruitment of interacting proteins (reviewed in (Litchfield, 2001)). One of these interaction partners is CKIP-1, which interacts with CK2 $\alpha$ but not with CK2 $\alpha^{\prime}$. CKIP-1 contains two protein-protein interaction domains, an $\mathrm{N}$ terminal pleckstrin homology (PH) domain and a C terminal leucine zipper motif (Fig. 9) (Bosc et al., 2000; Olsten ME, 2005). First, the $\mathrm{N}$ terminal $\mathrm{PH}$ domain is found to be responsible for its interactions with CK2 $\alpha$. The interaction between CKIP-1 and CK2 leads to the recruitment of the kinase to the plasma membrane (Litchfield, 2001). Previous studies indicate that deletion of the $\mathrm{PH}$ domain abolishes the interaction with $\mathrm{CK} 2 \alpha$. The PH domain of CKIP-1 binds to the HIKE domain of CK2 $\alpha$ (Olsten ME, 2005). HIKE is a conserved sequence motif, which was identified in strong $\mathrm{PH}$ domain interacting proteins such as, $\mathrm{G}$ proteins and protein kinases (Fig. 9) (Ciccarelli FD, 2000). HIKE contains five conserved residues (Asp, Leu, His, Lys, Glu, Gly), which are located in a $\beta$ strand-loop- $\beta$ strand within the HIKE motif (Fig. 9) (Litchfield, 2001). Although the overall structures of the kinases that contain this motif are quite different, the signature $\beta$ strand-loop- $\beta$ structural element is common in all of them (Fig. 9). After comparing this $\beta$ strand-loop- $\beta$ strand in different proteins, the lengths of the strands were found to be very similar but the angle between the two seems to be flexible 
(Olsten ME, 2005). Furthermore, there are various genetic and metabolic human diseases that are related to the mutations in this motif (Ciccarelli FD, 2000).

The interaction between CKIP-1 and CK2 raises the possibility that CKIP-1 might be acting as a non-enzymatic regulatory partner for CK2 by changing the localization of the enzyme to the plasma membrane. Therefore, CKIP-1 might shed light on how CK2 is regulated in vivo and raises the possibility of the presence of other CK2 interacting proteins. One striking coincidence is the common residues between HIKE motif and the amino acid substitutions of Timekeeper, $\mathrm{M}^{161} \mathrm{~K}$ and $\mathrm{E}^{165} \mathrm{D}$. The glutamic acid residue is also a part of the HIKE consensus sequence and found to be the only different residue between CK2 $\alpha$ and other HIKE motif containing proteins. Therefore, the possibility remains that the $E^{165} \mathrm{D}$ substitution in Tik might abolish the interactions between CKIP-1 and CK2. In addition, both Tik substitutions reside in the signature $\beta$ strand-loop- $\beta$ strand. Therefore, both substitutions in Tik might elicit loss of CKIP-1 interaction.

\section{Protein phosphatase 2A (PP2A):}

PP2A is another unique interaction partner of CK2 $\alpha$, but not CK2 $\alpha^{\prime}$ (Litchfield, 2001). It has been previously shown that the core dimer of PP2A, (catalytic $(C)$ and scaffolding subunit (A)) interact with $\mathrm{CK} 2 \alpha$ (Olsten ME, 2005). The same study also discovered that, this interaction was mediated through a sequence motif, HENRKL, which is conserved in every CK2 $\alpha$ isoform in all metazoan organisms (Fig. 10). In a study conducted with the SV40 t-antigen, it was found that this protein interacts with PP2A via the same HENR/KL motif (Heriche et al., 1997). A binding-deficient version of CK2 $\alpha$ was created during the same study via substituting three of the residues to Ala. The catalytic activity of CK2 $\alpha$ was found to remain the same, which indicated that the mutations didn't create a major structural strain to the enzyme. In addition, the recruitment of the $\beta$ subunit abolished the CK2 $\alpha-P P 2 A$ 


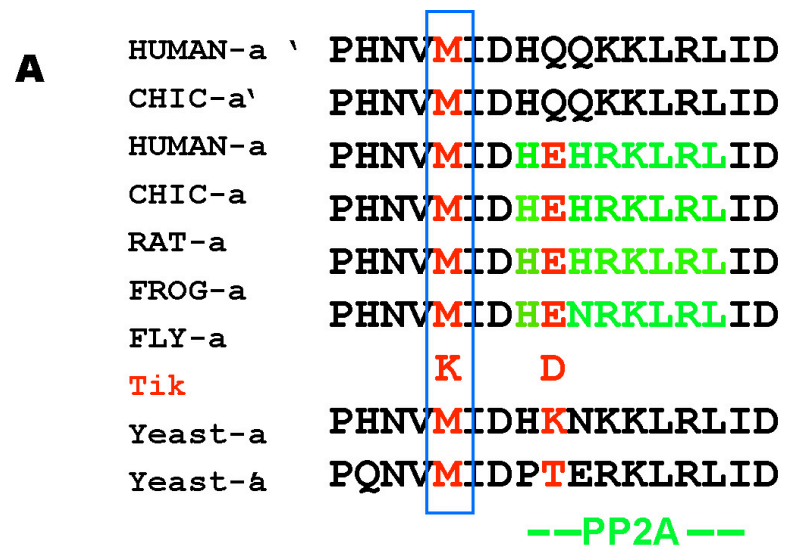

B

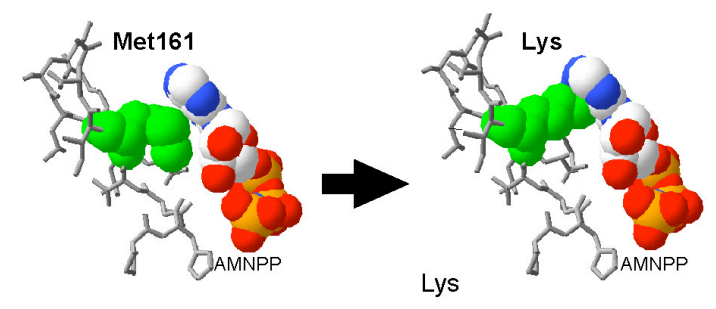

C


Figure 10: Amino acid substitutions in Tik. A) The sequence alignment representing the locations of $M^{161} K$ and $E^{165} D$ substitutions. The MK substitution is invariant unlike $E D$, which is only conserved in metazoans. The PP2A binding site is indicated in green, which colocolizes with the $E^{165} \mathrm{D}$ substitution. B) The MK substitution might disrupt ATP binding due to overlapping Van der Waals radia. C) The MK substitution might create internal structural clashes (purple) problems within the $\beta$-strand. D) The location of $E^{165}$ relative to the structure, which shows that it faces away from the active site. 
interaction, which suggests that PP2A perhaps only associates with $\mathrm{CK} 2 \alpha$, but not with the holoenzyme. This supports the idea that there are separate subpopulations of CK2 present in the cells, free $\mathrm{CK} 2 \alpha$ and the tetrameric holoenzyme. Another interesting finding that came out from this study is the fact that CK2 $\alpha$ could phosphorylate the PP2A catalytic subunit, a modification that appears to increase the activity of PP2A. In addition, the PP2ACK2 interaction also resulted in down regulation of MEK1 activity, which is regulated by PP2A (Lebrin F, 1999). Therefore, it is possible that CK2 is indirectly involved in this circuit byactivating PP2A. However, other in vivo roles of PP2A-CK2 complex still remain to be solved.

Another striking coincidence is the common residues between HENRKL motif and Tik. As stated previously, Tik contains two amino acid substitutions, $M^{161} \mathrm{~K}$ and $\mathrm{E}^{165} \mathrm{D}$. The $E^{165} \mathrm{D}$ substitution is part of the HENRKL motif. Therefore, it is possible that this substitution perturbs the interaction between CK2 and PP2A (Fig. 10). The relevance of this will be discussed later.

\section{HISTORY OF PROTEIN PHOSPHATASE SUPERFAMILY}

Reversible phosphorylation is a major mechanism used to regulate a wide variety of events. As mentioned before, one third of all proteins are regulated by this mechanism. Although there are hundreds of kinases, $>95 \%$ of which are Ser/Thr kinases, the number of phosphatases is not stoichiometric. It is becoming increasingly evident that kinases do not operate as isolated entities, but rather in supra-molecular complexes (Giot et al., 2003). These can include other proteins such as signaling scaffolds, inhibitors, partners and phosphatases. Phosphorylation is emphasized more than dephosphorylation as a regulatory mechanism, since our understanding of substrate specificities of protein kinases is more advanced than that for phosphatases. Like phosphorylation, dephosphorylation is a fundamental means to affect many cellular processes such as activation or deactivation, 
stabilization or destabilization and localization of key components. Although protein kinases are coming from a single gene family, protein phosphatases originate from three distinct gene families (reviewed in (Barford D, 1998)). The phosphatases that can remove the phosphate from Ser and/or Thr residues belong to PPM and PPP families. Dual specificity phosphatases and phosphatases that can dephosphorylate Tyr residues belong to PTP family. Ser/Thr phosphatases are divided further into two groups; type 1 and type 2 phosphatases. Type 2 phosphatases are Protein Phosphatase 2A (PP2A) (constitutively active), Protein Phosphatase 2B (PP2B) $\left(\mathrm{Ca}^{++}\right.$and calcineurin dependent) and Protein Phosphatase 2C (PP2C) $\left(\mathrm{Mg}^{++}\right.$dependent). For the interest of this work I will focus on the PPP family member, PP2A, for the rest of this discussion.

Catalytic domains of all PPP family members share similar architectural features, such as a $\beta$-sandwich $(\beta-\alpha-\beta-\alpha-\beta)$ structure within the active site located at the interface of the $\beta$ sheets (Barford D, 1998). These common elements allow different members of this family to have numerous overlapping targets in vitro. However, target specificity is achieved via different forms of regulation in vivo. For instance, the most abundant member, PP2A, has distinct regulatory subunits, which considerably increases the target specificity of the enzyme (Goldberg, 1999).

\section{Protein phosphatase 2A:}

PP2A is the most abundant member of Ser/Thr phosphatase PPP family in eukaryotes and is highly conserved from yeast to humans (Janssens and Goris, 2001). PP2A is involved in various signaling pathways, cell cycle progression, DNA replication, transcription and translation (reviewed in (Mumby MC, 1993)). In this discussion, I will focus on different aspects of PP2A: a) the composition and structure, b) molecular and genetic studies in model organisms, c) major roles, d) regulation. 


\section{A) Composition and structure:}

PP2A is a trimeric holoenzyme that comprises a structural A subunit, regulatory $B$ subunit and catalytic $\mathrm{C}$ subunit (Fig. 11) (Cho and Xu, 2007). The $65 \mathrm{kDa}$ A subunit (PR65) can form a dimer with the $36 \mathrm{kDa}$ catalytic $\mathrm{C}$ subunit (PP2Ac), which is named the 'core dimer' (Cho and $\mathrm{Xu}, 2007$ ). The various regulatory subunits can be recruited to this core dimer and form several versions of the trimeric holoenzyme. The association of the regulatory subunit is essential for enzyme activity. The B subunits are encoded by four different genes denoted by B, B', B', B'”, all of which are structurally unrelated. The high number of regulatory subunits is thought to provide a means to regulate this highly pleiotropic enzyme in vivo. It has been shown that association of the core dimer with different regulatory subunits changes the overall activity, substrate specificity and the subcellular localization of the holoenzyme.

The crystal structure of PP2A was a great interest for many labs for over 20 years; however, the crystallization of the protein was unexpectedly difficult. The structure was finally solved in 2006, by two independent labs concurrently (Cho and Xu, 2007; Xu et al., 2006). This provides insights into our understanding of various aspects of this complex enzyme and its functions.

\section{Structural A subunit (PR65):}

The structural (or scaffolding) A subunit is essential for the formation of the trimeric holoenzyme (Kobe B, 1999). The primary function of this subunit is to mediate the interactions between catalytic and various regulatory subunits. There are two isoforms, $\alpha$ and $\beta$, with $\% 86$ sequence identity. However, $\beta$ isoform is expressed in a limited manner, which restricts our understanding of this isoform. On the other hand, the sequence similarity among different species from human to yeast is quite low, with only 35\% (Kobe B, 1999). 
The crystal structure of PP2A-A was solved in 1999 by the laboratory of David Barford and revealed the presence of 15 tandemly repeated HEAT motifs (Fig. 11) (Groves MR, 1999; Kobe B, 1999). The HEAT motif was named after the proteins that share the same motif. These are Huntington's disease associated protein (Huntington), elongation factor 3 (EF3), A subunit of PP2A and a lipid kinase TOR1 (Kobe B, 1999). There are two a-helical loops in a single HEAT motif, which are named A-loop and B-loop. These loops are organized in a manner that A-loops face outward and B loops face inward in the overall structure of PR65 (Fig. 11). Although these proteins are not homologous, sequence analysis revealed that this tandemly repeated sequence plays a critical role functionally in various protein-protein interactions. HEAT motifs generally are $37-43$ residues in length, which form 3 to 22 tandem repeats (Groves MR, 1999; Kobe B, 1999). These repeats are formed with two antiparallel $\alpha$ helices with a weak consensus sequence in which hydrophobic residues are spaced with highly conserved Pro, Asp and Arg residues (Groves MR, 1999). Mutational analyses revealed that various repeats can interact with different proteins. For instance, repeats $11-15$ interact with the catalytic subunit, whereas repeats 2-8 interact with these regulatory subunits and viral proteins (Groves MR, 1999). These analyses also identified the residues that are altered during oncogenic transformations. For example, mutations that attenuate/disrupt binding of the $C$ and $B$ subunits are found in number of human lung and breast cancers (Groves MR, 1999).

\section{Regulatory B subunits:}

The four gene families that encode various regulatory subunits for PP2A are B (PR55), B' (B56 or PR61), B"' (PR72), and B'” (PR93/PR110) (Janssens and Goris, 2001). Each B subunit also has number of variations due to alternative splicing. This results in approximately 75 possible holoenzyme combinations. Although the different isoforms of $A$ and $C$ subunits are highly similar, the sequences amongst $B$ isoforms are quite different. 
$\mathbf{A}$

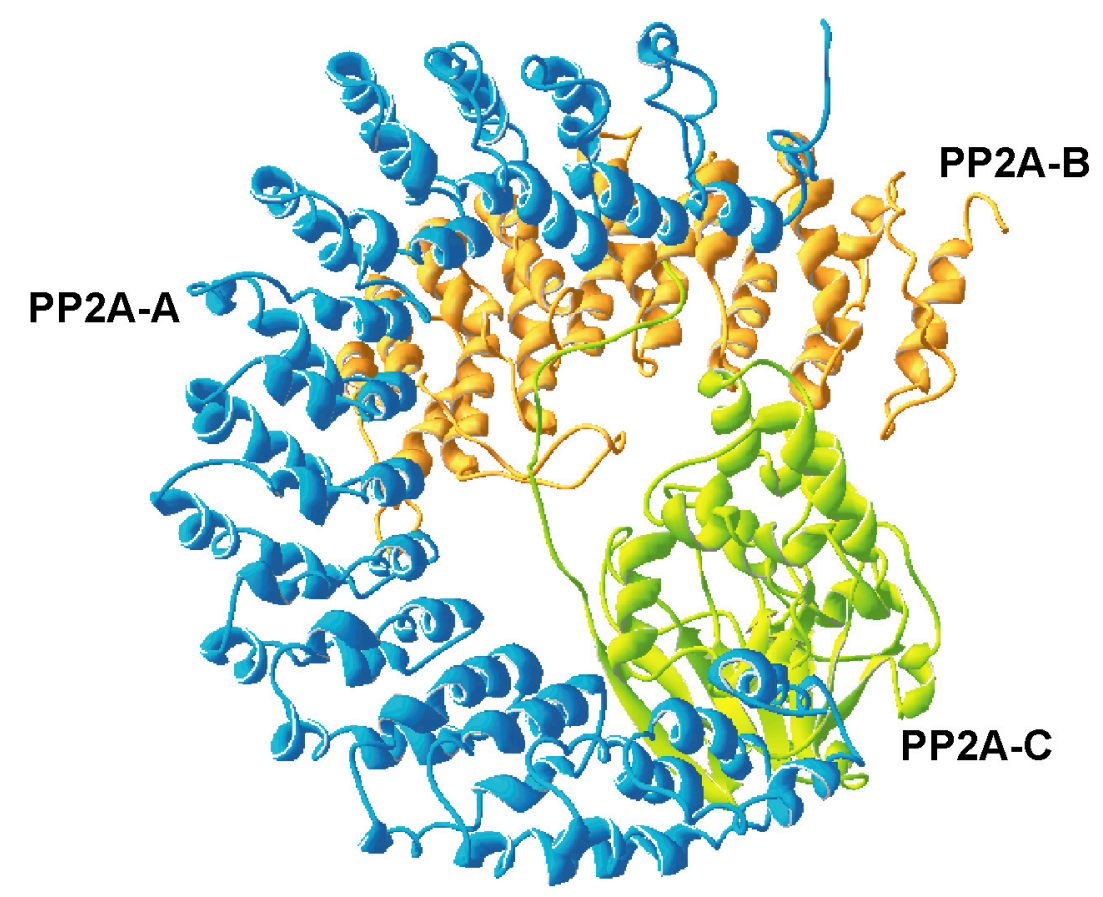

B
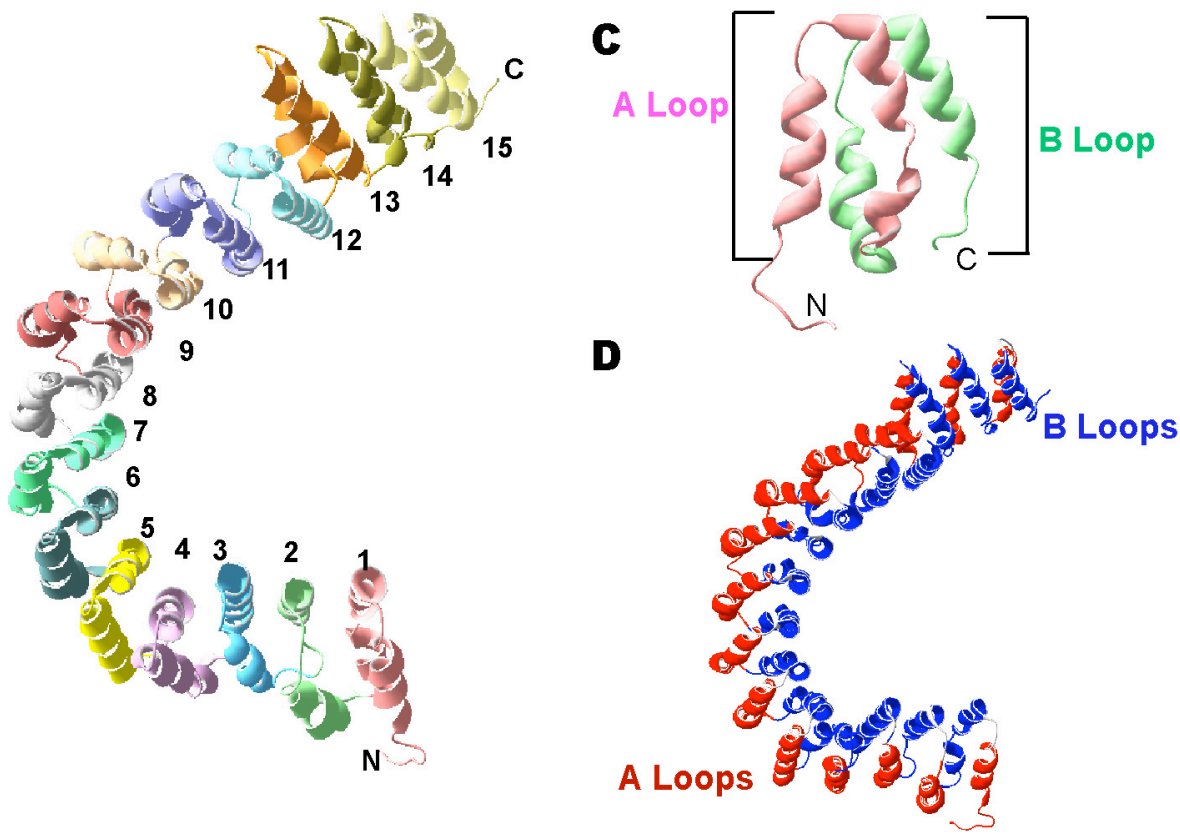

Figure 11: Structure of PP2A holoenzyme: A) Trimeric structure of the PP2A holoenzyme, the structural $A$ subunit (blue), the catalytic $C$ subunit (green) and regulatory $B$ subunit (orange), is shown in the figure ( $p d b$ file 2iae). B) The A subunit contains 15 tandem HEAT repeats. C) Each repeat is composed of two $\alpha$-helical loops (A (pink) and B (green)). D) A loops face outwards, unlike B loops, which face inward and interact with catalytic and regulatory subunits. 
The expression levels amongst these $B$ isoforms also differ in a cell, tissue or developmental specific manner (Xu et al., 2006). Therefore, the B subunit, in essence, establishes the specificity and localization of the trimeric holoenzyme. Since there are numerous isoforms of the regulatory subunit, the crystal structures of only a handful have been solved. For instance, the structure of B'/PR61 isoform revealed the presence of HEAT repeats in this subunit as well (Xu et al., 2006). Heat repeats 4-5 of the B' interact with HEAT repeats 2-5 of the A subunit. This interaction elicits a significant conformational change in the $A$ subunit. The B'/PR61 family is previously shown to play an essential role during cell-cycle progression, through interactions with basic proteins like Shugoshin (Rivera T, 2006).

\section{Catalytic C subunit (PP2Ac):}

There are two isoforms of the catalytic subunit of PP2A, $\alpha$ and $\beta$ that are $97 \%$ similar (Fig. 12) (Cho and Xu, 2007; Xu et al., 2006). The $\alpha$ isoform is predominantly expressed, as compared to the $\beta$ isoform(Cho and Xu, 2007; Xu et al., 2006). The structure and sequence of PP2Ac is highly conserved throughout evolution from Drosophila to human unlike the other subunits of PP2A (Fig. 12). The $\beta$-sandwich $(\beta-\alpha-\beta-\alpha-\beta)$ structure of PP2Ac is very similar to that of other phosphatases like PP1 and calcineurin. One important feature of PP2Ac is the presence of a methylation site at the $C$ terminus Leu ${ }^{309}$ (Longin S, 2007; Mumby, 2001). The reversible methylation is mediated via C-terminal leucine methyltransferase (CMT) and demethylation is mediated via PP2A-specific carboxymethyl esterase (CME) (Longin S, 2007). The significance of this event will be discussed later.

\section{B) Molecular and Genetic Studies in Model Organisms:}

\section{S. cerevisiae:}

As with CK2, budding yeast provided a powerful genetic system for studying the functions of PP2A in cell biology. PP2A is also found in a trimeric form in yeast, a scaffolding 


$$
\begin{aligned}
& \text { M. musculus } \\
& \text { D. rerio } \\
& \text { H. sapiens } \\
& \text { D. melanogaster } \\
& \text { S. cerevisiae }
\end{aligned}
$$

M. musculus

D. rerio

H. sapiens

D. melanogaster

S.cerevisiae

$$
\begin{aligned}
& \text { M. musculus } \\
& \text { D.rerio } \\
& \text { H. sapiens } \\
& \text { D.melanogaster } \\
& \text { S. cerevisiae }
\end{aligned}
$$

$$
\begin{aligned}
& \text { M. musculus } \\
& \text { D. rerio } \\
& \text { H. sapiens } \\
& \text { D. melanogaster } \\
& \text { S.cerevisiae }
\end{aligned}
$$

$$
\begin{aligned}
& \text { M. musculus } \\
& \text { D. rerio } \\
& \text { H. sapiens } \\
& \text { D. melanogaster } \\
& \text { S.cerevisiae }
\end{aligned}
$$

$$
\begin{aligned}
& \text { M. musculus } \\
& \text { D. rerio } \\
& \text { H. sapiens } \\
& \text { D.melanogaster } \\
& \text { S. cerevisiae }
\end{aligned}
$$

MDDKAFTKELDOWVEQLNECKOLNENOVRTLCEKAKE ILTKESNVOEVRCPVTVCGDVHG MDDKAFTKELDOWVEQLNECKOLTENQVRTLCEKAKE I LTKESNVQFVRCPVTVCGDVHC MDEKVFTKELDOWI EQLNECKOLSESQVKSLCEKAKEILTKESNVQEVRCPVTVCGDVHC MEDKATTKDLDQWIEQLNECNQLTETQVRTLCDKAKE ILSKESNVQEVKCPVTVCGDVHC $--T N T N I N O L D O W I$ EHLSKCEPLSEDDVARLCKMAVDVLOFEENVKP INVPVTICGDVHG

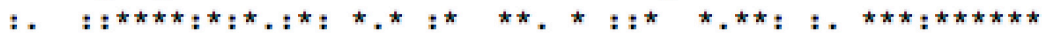

QFHDLMELFRICGKSPDTNYLFMGDYVDRGYYSVETVTLLVALKVRYPERITILRGNHES QFHDLMELFRICGKSPDTNYLFMCDYVDRGYYSVETVTLLVALKVRYPERITILRGNHES QFHDLMELFRIGGKSPDTNYLFMGDYVDRGYYSVETVTLLVALKVRYRERITILRGNHES QFHDLMELFRIGGKSPDTNYLFMGDYVDRGYYSVETVTLLVALKVRYRERITILRGNHES QFHDLLELFKICGPCPDTNYLFMCDYVDRCYYSVETVSYLVAMKVRYPHRITILRGNHES

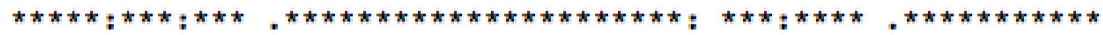

RQITQVYGFYDECLRKYGNANVWKYFTDLFDYLPLTALVDGQIFCLHGGLSPSIDTLDHI ROITQVYGFYDECLRKYGNANVWKYFTDLFDYLPLTALVDGQIFCLHGGLSPSIDTLDHI RQITQVYGF YDECLRKYGNANVWKYFTDLFDYLPLTALVDGQIFCLHGGLSPSIDTLDHI ROITQVYGFYDECLRKYGNANVWKYFTDLFDYLPLTALVDGQIFCLHGGLSPSIDSLDHI RQITQVYGF YDECLRKYGSANVWKMFTDLFDYF PVTALVDNKIFCLHGGLSPMIETIDQV

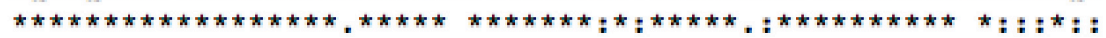

RALDRLQEVPHEGPMCDLLWSDPDDRGGWGISPRGAGYTFGQDISETFNHANGLTLVSRA RALDRLQEVPHEGPMCDLLWSDPDDRGGWGISPRGAGYTFGODISETFNHANGLTLVSRA RALDRLOEVPHEGPMCDLLWSDPDDRGGWGISPRGAGYTFGODISETFNHANGLTLVSRA RALDRLOEVPHEGPMCDLLWSDPDDRGGWGISPRGAGYTFGODISETFNNTNGLTLVSRA RDLNRIQEVPHEGPMCDLLWSDPDDRGGWGISPRGAGFTFCQDISEQFNHTNDLSLIARA

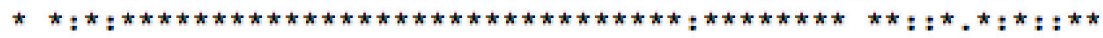

HOLVMEGYNWCHDRNVVTIFSAFNYCYRCGNQAAIMELDDTLKYSFLQFDPAPRRGEPHV HQLVMEGYNWCHDRNVVTIFSAFNYCYRCGNOAAIMELDDTLKYSFLOFDPAPRRGEPHV HQLVMECYNWCHDRNVVTIFSAFNYCYRCGNQAAIMELDDTLKYSFLQFDPAPRRGE PHV HQLVMEGYNWCHDRNVVTIFSAPNYCYRCGNOAALMELDDSLKF SFLQFDPAPRRGEPHV HQLVMEGYSWSHQONVVTIFSAPNYCYRCGNQAAIMEVDENHNRQFLQYDPSVRPGEPTV

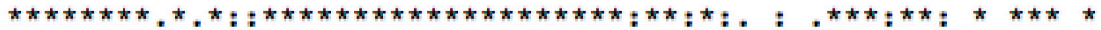

TRRTPDYFL TRRTPDYFL TRRTPDYFL TRRTPDYFL TRKTPDYFL $\star \star \vdots: * \star \star \star * * \star$

Figure 12: Alignment of PP2A catalytic subunits: The highly conserved PP2A catalytic subunits represents $97 \%$ sequence identity. The alignments were created with ClustalW. 
A subunit, regulatory $B$ subunit and catalytic $C$ subunit. The scaffolding $A$ subunit is encoded by a single gene, TPD3, whose deletion renders cells to become cold or temperature sensitive (Jiang, 2006). Regulatory subunits are encoded by two distinct genes, CDC55 and RTS1, which are homologs of $\mathrm{B}$ and $\mathrm{B}$ ' isoforms in mammals, respectively (Jiang, 2006). The B subunits are not essential for viability; however, their deletion makes yeast stress-sensitive. The catalytic $C$ subunit is encoded by two genes, PPH21 and PPH22, which are almost identical (Jiang, 2006). There is no prominent effect upon deletion of either gene. However, deletion of both genes diminishes PP2A activity by $90 \%$ and severely retards cell growth (Janssens and Goris, 2001). There is a third gene, PPH3, which is responsible for the remaining $10 \%$ activity, which encodes for a structurally similar phosphatase. Deletion of all three is found to be lethal (Janssens and Goris, 2001). There are other enzymes in yeast that exhibit structural similarities to PP2A, which are named 2A-like phosphatases (Jiang, 2006). These enzymesare also involved in cell cycle progression and cellular growth.

\section{D. melanogaster:}

Unlike yeast and mammals, only some of the components of PP2A have been characterized in Drosophila to date. These are the structural subunit PR65, catalytic subunit PP2Ac and two of the regulatory subunits PR55 (B) and PR61 (B') (Janssens and Goris, 2001). Although PP2A is expressed throughout Drosophila development, the expression levels change in a tissue- and time-specific manner (Janssens and Goris, 2001). First, PR65 scaffolding subunit levels increase during early developmental stages, especially in the brain and imaginal discs during third instar larvae. The levels of PP2Ac and PR65 appear to be similar, and both proteins colocalize to the same regions, consistent with the possibility that they are found as a core dimer in vivo (Janssens and Goris, 2001). Therefore, levels of PP2Ac are also high during early development 


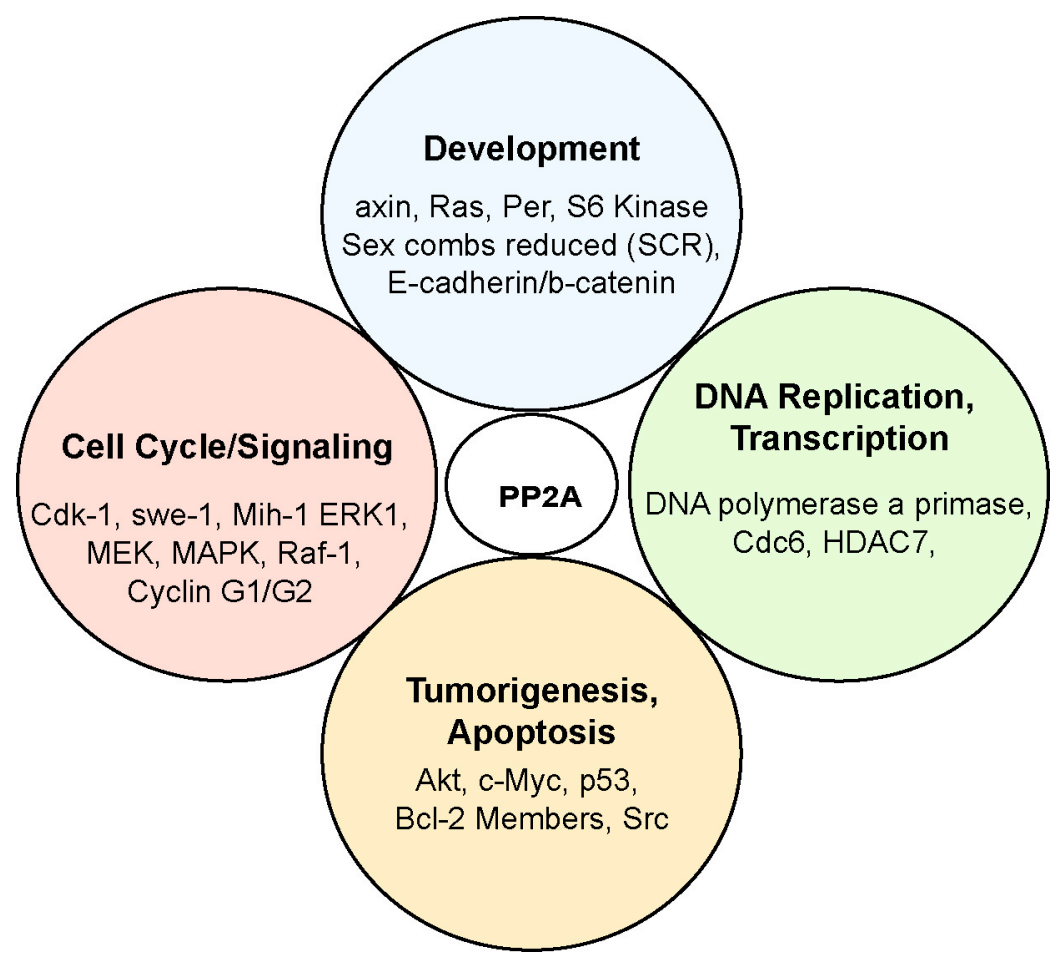

Figure 13: Global roles of PP2A: PP2A is a highly pleitropic enzyme, which is expressed ubiquitously. It is involved in a wide variety of cellular processes, some of which are sorted into groups. These targets represent only a small portion and have been identified by direct dephosphorylation in vitro/in vivo. 
(Janssens and Goris, 2001). The mutants for PP2Ac are unable to complete embryogenesis and exhibit cell cycle defects. There are several phenotypes associated with these mutants related to chromatin structure (Gurland G, 1993). First, the mutants display disorganized chromatin structure with multiple centromeres. It has been found that PP2A is required for microtubule attachment to the DNA during anaphase. These mutants are named 'microtubule star (mts)' because arrays of microtubules radiate from these multicentromeric structures, which look similar to a 'star' (Gurland G, 1993). Accordingly, mutants for PR55 also display mitotic defects, which are called abnormal anaphase resolution (aar1) and twinsP (Mayer-Jaekel RE, 1993; Uemura T, 1993). In aar1 mutants, chromosomes do not get separated during anaphase and stay intact. However, these defects can be rescued by reintroduction of PR55. On the other hand, in the twinsP mutants, the wing imaginal discs develop abnormally, with a mirror image, which reveals the important role of PP2A during tissue specification and in establishing tissue asymmetry (Uemura T, 1993).

\section{C) Major targets and roles of PP2A:}

PP2A is a highly pleiotropic enzyme, which causes this enzyme to be involved in virtually all aspects of cell biology. PP2A has number of targets, which are involved in numerous signaling networks and gene expression/transcription. The global functions of PP2A are summarized in the Figure 13. The major cell autonomous roles that will be discussed in the following pages include cell cycle, cell survival and apoptosis. The major cell specific roles include Wnt signaling, circadian clock and EGFR signaling.

\section{1) Cell autonomous roles:}




\section{Cell cycle and apoptosis:}

As with protein kinases (see above), protein phosphatases such as PP1 and PP2A are also involved in various stages of the cell cycle (Janssens and Goris, 2001). Inhibition of PP1 and PP2A by inhibitory agents, such as Okadaik acid (OA) elicited tumor formation and early entry into mitosis, indicating that these enzymes function as tumor suppressors and negative regulators of the cell cycle (Haystead TA, 1989). However, it is important to study the individual regulatory subunits of these phosphatases since complete inhibition of these enzymes elicit a wide range of defects, which makes it harder to pinpoint a cellular role. Specifically, PP2A is found to block the entry into $S$ and $M$ phase transitions via deactivating Cdk-1 (Janssens and Goris, 2001; Jiang, 2006). In higher eukaryotes, Cdk-1 activity increases in PP2A mutants, which leads to early mitotic entry (Wang Y, 2005). However, inactivation of the catalytic subunit in conditional mutations of $S$. cerevisiae leads to cell cycle arrest at G2/M. Furthermore, PP2A is also involved in cytokinesis, as inactivation of the regulatory subunits result in multinucleated cells (Jiang, 2006).

\section{Viral transformation:}

DNA tumor viruses such as SV40, among others, take advantage of the pleiotropic nature of PP2A upon entering the host cell (reviewed in (Arroyo and Hahn, 2005)). The AC core dimer is a target for these viruses by displacing the endogenous B subunit and changing the target specificity of the holoenzyme. Only SV40 small t-antigen will be discussed in this portion.

PP2A is the only known cellular target for SV40 small t-antigen, which belongs to Simian virus (SV) 40 DNA viruses family (Arroyo and Hahn, 2005). This virus is especially become important since it has the ability to cause oncogenic transformation. This virus can interact with the AC core dimer by binding to HEAT repeats 3-5 of the A subunit (Cho et al., 
2007). Under normal circumstances, the regulatory subunit binds to this region; therefore, the small t-antigen mimics the regulatory subunit and changes the target specificity of the enzyme towards cellular growth proteins such as ERK1, MEK and PKC (Sontag et al., 1993).

\section{2) Cell specific/developmental roles:}

\section{Wnt signaling:}

As described above, ultimate result of Wnt canonical pathway is the accumulation of $\beta$-catenin in the nucleus, which activates the transcription of $\beta$-catenin sensitive genes (Polakis, 2000). Like CK2, PP2A also plays its part during this pathway via dephosphorylating the axin component of the $\beta$-catenin destruction complex (Ratcliffe MJ, 2000). Dephosphorylation of axin makes this complex instable, thus, PP2A plays a suppressive role in this pathway (Ratcliffe MJ, 2000).

\section{EGFR signaling:}

Epidermal growth factor receptor (EGFR) pathway is another canonical pathway, which is used extensively during development of an organism. For instance, EGFR pathway is involved in neuronal specification of photoreceptors during eye development, which is well studied in Drosophila (Flores et al., 2000; Price et al., 1997). In brief, activation of EGFR leads to the activation of the Ras/Raf/MEK/MAPK cascade and PP2A dephosphorylates multiple components of this pathway, such as Ras (Wassarman et al., 1996). It has been found that PP2A mutants display increasing number of cellular transformations, which are linked with constitutively activated form of Ras (Wassarman et al., 1996). 


\section{Circadian clock:}

As discussed above, posttranslational events play a crucial role for the integrity of the circadian clock. Two autoregulatory feedback loops mediate the expression of the central clock proteins such as Period (PER) (Allada, 2003). The timely degradation and accumulation of this protein in the cytoplasm seems to mediate the rhythm of the clock. Posttranslational modifications such as phosphorylation and dephosphorylation are critical for these events. PER is phosphorylated via number of kinases including CK2 and becomes susceptible for degradation by the E3 ligase SLIMB (Lin et al., 2002). This degradation is counteracted via dephosphorylation by PP2A, which stabilizes PER and enables association with another clock protein, Timeless (TIM) (Sathyanarayanan et al., 2004). This accumulation, during the day, allows these proteins to reach a critical concentration in the cytoplasm, which is followed by nuclear translocation. The nuclear PER and TIM complex, in turn, inhibits the actions of $c / k$ and cyc genes (Lin et al., 2002). Therefore, antagonistic actions of PP2A and CK2 seem to play an important role for cycling of the PER, which ensures the rhythm of the clock.

\section{D) Regulation of PP2A:}

It is surprising that there are far less number of phosphatases when compared to the huge number of kinases. The overall regulation of the phosphatases, thus, becomes an interesting and crucial topic to study. PP2A is one of the well-studied phosphatases and a lot has been learnt over time about the roles within the cell. However, the regulation of PP2A is still enigmatic. The possible methods of regulation include: 1) the expression and assembly of the holoenzyme, 2) posttranslational modifications such as phosphorylation and methylation, and 3) small molecules. 


\section{1) Expression/assembly of the holoenzyme:}

As mentioned before, there are 75 possible holoenzyme combinations for PP2A, which raises the possibility of regulation at the level of holoenzyme expression/assembly (Goldberg, 1999). The large number of regulatory subunits are expressed in a tissuespecific, cell-specific or developmentally-specific manner. Thus, this raises the possibility of diverse sub-populations of PP2A in different regions of the cell/tissue at different time points during development.

\section{2) Posttranslational modifications:}

Phosphorylation and methylation are the two major posttranslational modification events that are involved in regulation of PP2A. The catalytic subunit PP2Ac and various regulatory subunits are phosphorylated via number of kinases. There are two distinct phosphorylation sites on the catalytic subunit, $\mathrm{Tyr}^{307}$ and a Thr residue whose location remains to be discovered. (Goldberg, 1999) The phosphorylation of $\mathrm{Tyr}^{307}$ is mediated via tyrosine kinases, pp60 and pp56, which blocks the activity of the catalytic subunit (Goldberg, 1999). The amount of phosphorylated form of PP2Ac seems to increase in response to growth stimulation via epidermal growth factor or serum (Janssens and Goris, 2001). As stated before, PP2A negatively regulates the cell cycle; thus, inactivation of PP2A via phosphorylation could allow for growth. Most regulatory subunits are also subject to reversible phosphorylation. Especially the B' family members are found to undergo this mode of regulation such as PR61a, which is phosphorylated by double stranded RNA dependent protein kinase (PKR) $(X u Z, 2000)$. This seems to change the substrate specificity of the enzyme.

Like phosphorylation, methylation is another posttranslational event that is involved in regulation. As mentioned above, methylation of PP2Ac by C-terminal leucine 
methyltransferase (CMT) seems to be essential in some cases (Mumby, 2001). The loss of methyltransferase activity is found to prevent the formation of the holoenzyme in yeast (Longin S, 2007). However, C terminal truncated versions of PP2Ac, which are lacking the entire $\mathrm{C}$ terminal region, can also form the trimeric holoenzyme (Cho and $\mathrm{Xu}, 2007)$. It is possible that methylation is necessary for recruitment of certain regulatory subunits (Longin S, 2007).

\section{3) Small molecules:}

Small molecules like second messengers are involved in regulation such as the lipidlike second messenger ceramide. Ceramide interaction plays a role during apoptosis via inactivating Bcl-2, an antiapoptotic protein (Ruvolo PP, 1999). Another small molecule that can interact with PP2A and is highly used during molecular studies is OA (Haystead TA, 1989; Xing et al., 2006). OA inhibits most of the Ser/Thr phosphatases including PP2A thorough binding to the catalytic subunit. As mentioned before, inhibition of PP2A via OA results in tumor formation (Haystead TA, 1989). 


\section{RATIONALE AND HYPHOTHESES OF THIS WORK}

CK2 and PP2A are two enzymes that are involved in various cellular processes, but the relevance of one to the other is presently unclear. This section describes pertinent aspects of $\mathrm{N}$ signaling with emphasis on neurogenesis, since the development and patterning of the compound eye and mechanosensory bristles is the basis of the work described here.

\section{The role of Notch during neurogenesis:}

As stated before, $\mathrm{N}$ signaling plays an essential role during neural cell-fate determination during multiple stages of development both in invertebrates and vertebrates (reviewed in (Blaumuller and Artavanis-Tsakonas, 1997; Bray, 1998; Mumm and Kopan, 2000). This pathway is best knows for its role during 'lateral inhibition', a process in which a single cell from a group of equipotent cells alter the fate of its neighbors thorough the actions of $\mathrm{N}$. Thus, $\mathrm{N}$ signaling ensures the cellular diversity and prevents cells to gain identical fates. The default fate for the cells in the neuroectoderm is to become a neuron, however, signaling circuits like $\mathrm{N}$, selects only a certain number cells to gain a neural fate. The process of selecting a single cell from a group of cells is termed lateral inhibition, which involves $\mathrm{N}$ signaling (Artavanis-Tsakonas et al., 1995). The ability of $\mathrm{N}$ signaling to repress neuronal fate depends on the expression of $E(s p l)-b H L H$ repressors. The $E(s p /)$ locus encodes for seven basic helix loop helix (bHLH) proteins (M $\alpha, M \beta, M \delta, M 3, M 5, M 7, M 8)$ and a non bHLH protein Gro (Fig. 14) (Delidakis and Artavanis-Tsakonas, 1991). These proteins all share similar structural features. There are four functional domains, which are a basic domain, a helix-loop-helix domain, an orange domain and $\mathrm{C}$ terminal domain (Fig. 14) (Dawson et al., 1995). First, the basic domain mediates DNA-binding. The helix loop helix domain is responsible for homo/hetero dimerization amongst different members. Thirdly, the orange domain mediates the interactions with proneural proteins such as Ato or 


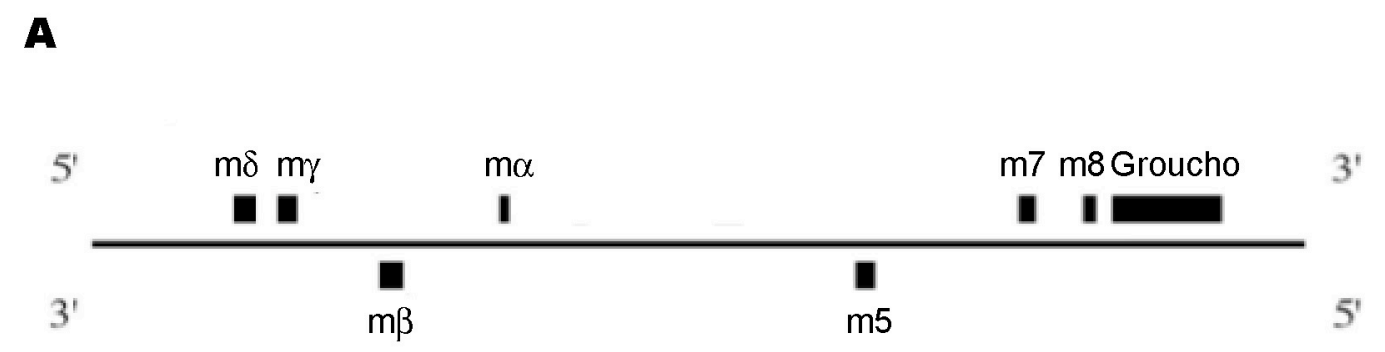

B

E(spl)M8 1130

C

CK2 Phosphorylation Site

M5

萡

M7 SISPVSSGYASDNESLLQISSP

M8 PLSPASSGYHSDCDSPPPTPQP

Figure 14 The Enhancer of split Complex: A) $E(s p /) C$ locus encodes for seven bHLH proteins $(\mathrm{M} \alpha, \mathrm{M} \beta, \mathrm{M} \delta, \mathrm{M} 3, \mathrm{M} 5, \mathrm{M} 7, \mathrm{M} 8)$ and a non-bHLH Gro, which are all located on the third chromosome (96F8-96F10). B) The schematic of the four functional domains found in E(spl)M8 which are: 1) bHLH domain (pink), 2) Orange domain (blue), 3) CK2 phosphorylation site (blue) and 4) the C-terminal domain (groucho interaction site WRPW) (green). C) Sequence alignment of M5, M7 and M8 that shows the conserved CK2 phosphorylation site (the phosphoacceptor Ser (red)). 
Acheate/scute proteins (Dawson et al., 1995). Lastly, the C terminal domain contains a WRPW motif, which mediates the binding of Gro. Gro recruitment is required for $\mathrm{E}(\mathrm{spl})$ proteins to become active repressors (Chen and Courey, 2000; Paroush et al., 1994).

During neural specification, bHLH proteins encoded by achaete-scute complex (ASC) and atonal (ato) are expressed in proneural clusters (PNCs) as a prelude to neural specification (Artavanis-Tsakonas et al., 1995, Baker, 1996 \#1152). Although, at this point, each cell has the potential to adopt a neural fate, only a fixed number of cells do so. This process is termed as lateral inhibition and involves $\mathrm{N}$ signaling (Fig. 15). This restriction is mediated when one cell of the PNC, which is called the future sensory organ precursor (SOP), expresses the highest levels of ASC or Ato and it, in turn, prevents its neighbors from adopting a similar, neural, fate (Artavanis-Tsakonas et al., 1999). First, the future SOP expresses the $N$ ligand $D I$. The interaction between the ligand DI and its receptor $N$ result in the cleavage and release of the Notch Intra-Cellular Domain $\left(\mathrm{N}^{\mathrm{ICD}}\right)$, which shuttles into the nucleus. Subsequently, association between the $\mathrm{N}^{\mathrm{ICD}}$ and DNA transcription factor $\mathrm{Su}(\mathrm{H})$ switches on $\mathrm{E}(\mathrm{spl})$ expression in the all other cells of the PNC (Ligoxygakis et al., 1998). These $E(s p l)$ repressors serve to block the neural fate by antagonizing the activities of ASC/Ato (Fig. 15) (Baker et al., 1996).

$\mathrm{N}$ signaling pathway is involved in eye and bristle development, both of which require $\mathrm{N}$ for proper number, positioning and pattern. In the case of the eye development, $\mathrm{N}$ signaling is required to for the selection of R8 founder cell during lateral inhibition. However, in the case of bristle development, $\mathrm{N}$ signaling is also required for sister cell fate specification besides SOP selection. The actions of $\mathrm{N}$ will be discussed in detail below in the eye and bristle, respectively. 
A

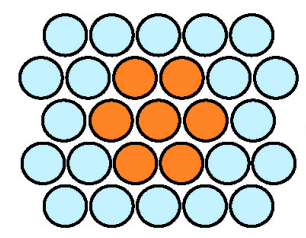

Neural Enhancement (Proneural Clusters)

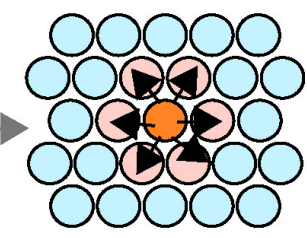

Lateral inhibition (Notch signaling)

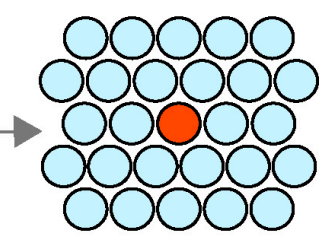

SOP/R8 Selection

B

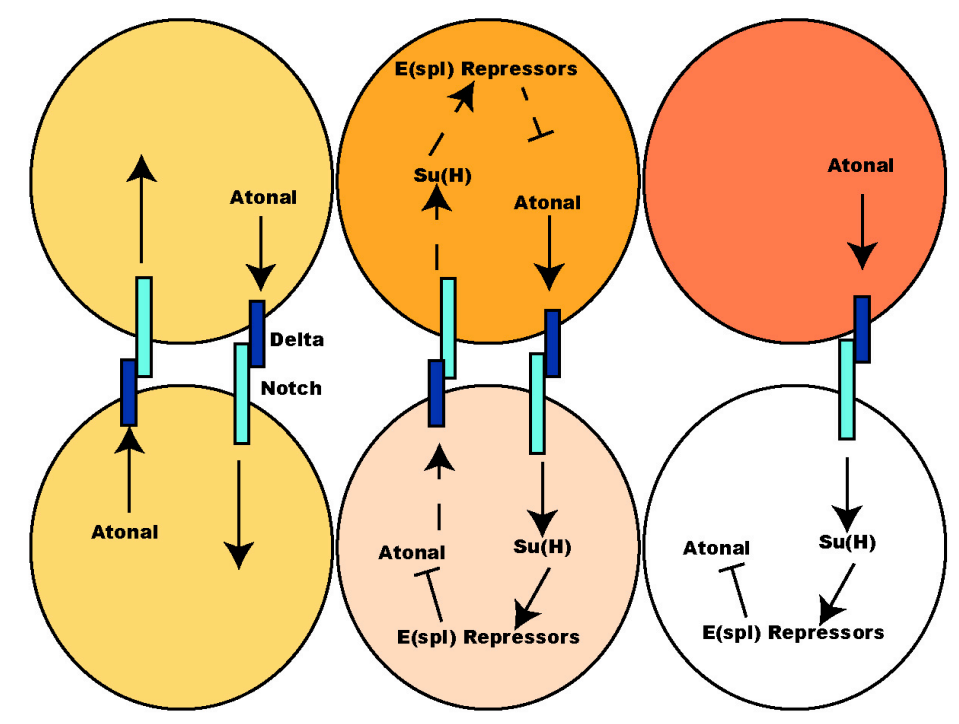

C

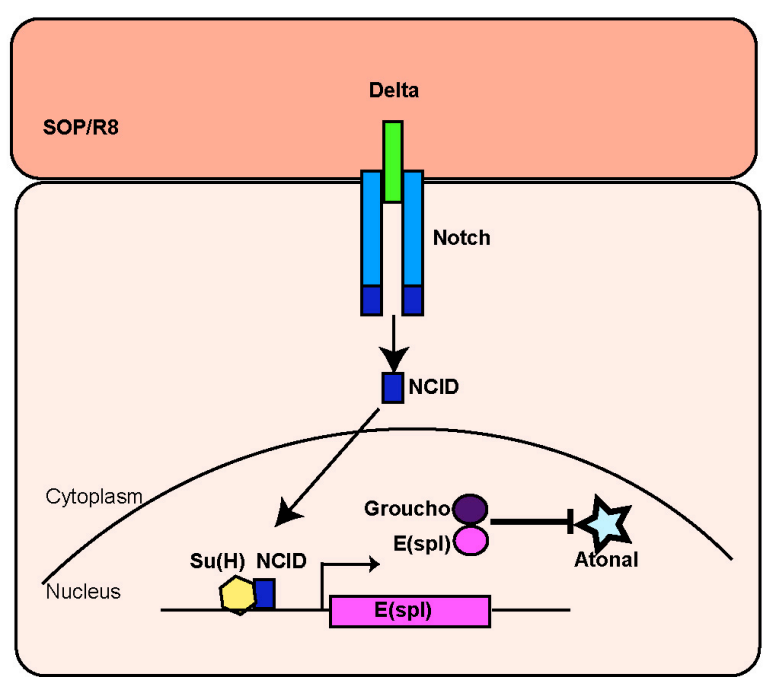

Figure 15: Notch mediated Lateral Inhibition: A) The selection of a single SOP/R8 (red) cell from a group of equipotent cells (proneural cluster) (orange), which is mediated by inhibitory actions of Notch (arrows). B) Important steps of Notch signaling, in Drosophila: 1) Interaction between DI (green) and $\mathrm{N}$ (blue) leads to the proteolysis of $\mathrm{N}^{\mathrm{ICD}}$ (dark blue). 2) $\mathrm{N}^{\mathrm{ICD}}$ translocation into the nucleus. 3) $\mathrm{N}^{\mathrm{ICD}}$ interaction with $S u(H)$ (yellow). 4) Activation of $E(s p l) C$ proteins (pink). The $E($ spl) proteins require Gro (purple) recruitment to repress the actions of Ato (light blue). 
The Drosophila eye is a widely utilized model to decipher the mechanisms of neural differentiation. Over the years, the studies in this system provided a great amount of information regarding the cell-fate specification, cell-cell communication and tissue patterning. Drosophila adult compound eye comprises 800 ommatidia, light sensing structures, with a precise hexagonal architecture. There are total of eight types of photoreceptors (R1-R8) and various supporting cells such as cone cells and pigment cells, which are responsible for different aspects of vision (Ligoxygakis et al., 1998). A single cell, $\mathrm{R} 8$, is selected from a group of cells in the PNC in the eye imaginal disc of third instar larvae (Fig. 16) (Ligoxygakis et al., 1998). This selection process is mediated in the 'morphogenic furrow (MF)', a wave of differentiation, which sweeps through the eye disc from anterior to posterior (Ma and Moses, 1995). This selection process is mediated via intricate signaling circuits involving Notch, EGFR among others. R8 selection is followed by recruitment of R2/R5, R3/R4 and R1/R6 photoreceptors in a pair wise manner (Hsiung and Moses, 2002). $\mathrm{R} 7$ is the last photoreceptor to be recruited, which completes the ommatial structure. Since $\mathrm{R} 8$ is the founder cell, and it orchestrates the recruitment of the remaining cells, any perturbations during its selection elicits loss of patterning of the compound eye, named 'rough eye'.

Bristles are mechanosensory organs that can be found as macro-chaetaes or microchaetaes. A single cell, SOP, is selected from a group of equipotent cells in the PNC in the wing disc of the larvae (Bose et al., 2006). There are two cell divisions that SOP undergoes to give rise to four cell types, neuron, sheat, shaft and socket (Fig. 16) (Bose et al., 2006). These cell divisions are all under the control of $\mathrm{N}$ signaling for proper cell type formation, number and location of the bristles. Therefore, compromising $\mathrm{N}$ activity elicits hyperplasia (ectopic and duplicated bristles) (Bose et al., 2006). In contrast, overexpression of $\mathrm{N}$ elicits missing bristles, split bristles and ectopic socket cell formation (Bose et al., 2006). 


\section{A}

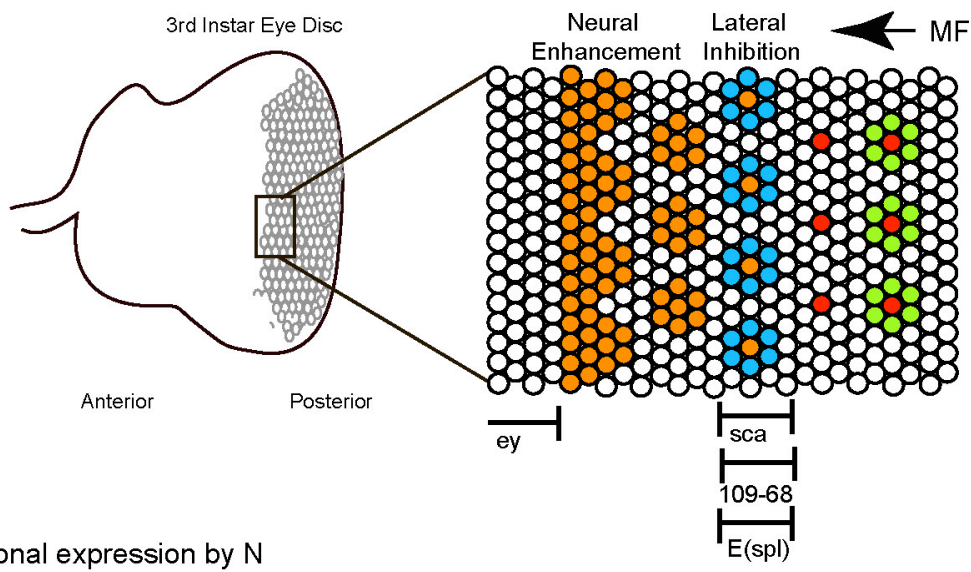

Lateral inhibition by $N$ \& $E(s p l)$

O R8 fate

Other R-cell fate

B

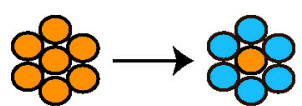

PNC
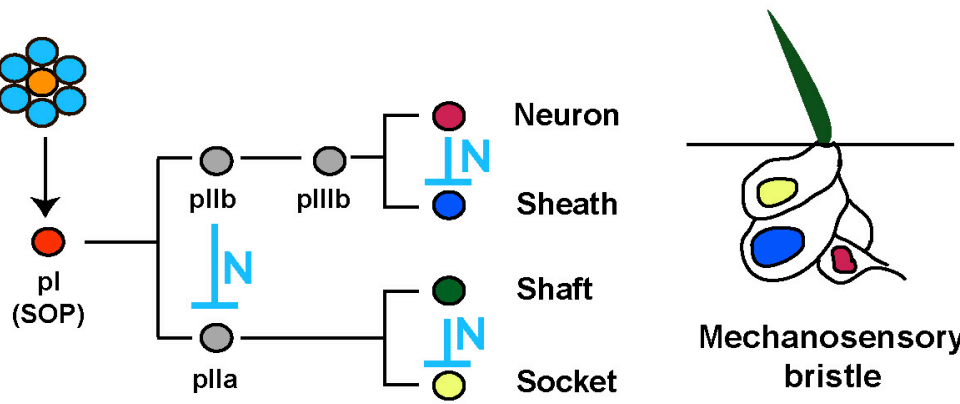

Mechanosensory bristle

\footnotetext{
O Achaete expression

Lateral inhibition by $N$ \& $E(s p l)$

O SOP fate
}

Figure 16: Role of Lateral Inhibition during eye and bristle morphogenesis: A) Proneural cluster of cells express Ato (orange) in the third instar larvae eye imaginal disc. Arrow indicates the movement of the morphogenetic furrow (MF), which selects only the R8 founder cell during lateral inhibition which is mediated by N. B) Like in the case of R8 selection, lateral inhibition plays a role during SOP selection. Notch is also involved in selection of the sister cell fates and specify different cell types that constitute a bristle which are neuron (pink), sheath (dark blue), shaft (green) and socket (yellow). 
The two general questions that are addressed in this work are the underlying reasons for the dominant behavior of Tik and potential antagonistic roles of PP2A and CK2 in $\mathrm{N}$ signaling pathway. Drosophila eye and bristle development will be employed as a model system while addressing these questions.

\section{Rationale \#1:}

Tik harbors two amino acid substitutions, $M^{161} \mathrm{~K}$ and $\mathrm{E}^{165} \mathrm{D}$. The former substitution is thought to mediate the dominant behavior since it lies in the ATP binding pocket (Lin et al., 2002). In contrast, the latter substitution has been thought to be silent and not contribute to the dominant behavior of Tik. However, the $E^{165} D$ mutation resides with the PP2A binding site, the HEN/HRKL motif that is shared between CK2 $\alpha$ and SV40 small t antigen (Heriche et al., 1997). Therefore, I hypothesize that Tik might be a double hit with both disrupted ATP binding and loss of regulation due to missing/altered PP2A binding. To test this hypothesis, two variants of CK2 $\alpha$ were generated, the first harboring only the MK substitution while the second the ED substitution. These variants then were used in a systematic manner to assess the contribution of each residue. I have employed complementation of yeast harboring null mutations in CK2 $\alpha$ subunits. The variants were also analyzed for protein-protein interactions and subjected to molecular modeling. Finally, the variants were analyzed in vivo in Drosophila with emphasis on neurogenesis as well as rescue of lethality in wild type and/or $N^{\text {spl }}$ flies.

\section{Specific Aims:}

1) Constructs were tested for their ability to complement the lethality of yeast harboring disruptions of the CKA1 and CKA2 genes.

2) The protein-protein interactions were tested for $\mathrm{CK} 2 \alpha$ variants against CK2 $\beta$.

3) Molecular modeling was employed to provide insights into the CK2 $\alpha$ variants. 
4) $\mathrm{CK} 2 \alpha-\mathrm{MK}$ and $\mathrm{CK} 2 \alpha-\mathrm{ED}$ constructs were introduced into the Drosophila germline to create independent transgenic lines, and tested for their ability to elicit dominant neural defects.

5) UAS-CK2 $\alpha-M K$ and UAS-CK2 $\alpha-E D$ lines were tested for their ability to suppress the neural defects of $N^{\text {spl }}$.

\section{Rationale \#2:}

It is increasingly becoming apparent that dynamic control of phosphorylation and dephosphorylation underlies the proper spatial and temporal activities of many signaling circuits including $\mathrm{N}$ pathway. Emerging evidence suggests that protein kinase CK2 mediates the effects of $\mathrm{N}$ during Drosophila eye and bristle development via phosphorylation of $\mathrm{E}(\mathrm{spl})$ repressors. Previously, it was shown in our laboratory that expression a dead catalytic subunit of CK2 (a CK2 $\alpha-D N$ ) elicits neural defects in the eye and bristle, which are similar to those upon loss of $E(s p l)$ (Bose et al., 2006). These results suggest that impaired CK2 function compromises lateral inhibition, presumably due to hypo-phosphorylation of $\mathrm{E}(\mathrm{spl}) \mathrm{M} 8$. The presence of phosphorylation during $\mathrm{N}$ signaling raises the possibility of dephosphorylation as a means to regulate of $\mathrm{E}(\mathrm{spl})$ proteins or other aspects of $\mathrm{N}$ signaling. Therefore, I hypothesize that PP2A might act in an antagonistic fashion to CK2 during N signaling. To test this hypothesis, I have employed Gal4-UAS system to assess the role of PP2A during eye and bristle development in wild type and/or $N^{\text {spl }}$ flies I have used three different UAS-lines that alter the activity or levels of the catalytic subunit of PP2A in different ways. The UAS-lines that are used during this study are: 1) overexpression line UAS-mts, 2) dominant negative line UAS-Dnmts, and 3) RNA interference (RNAi) line UAS-mtsRNA. These lines were analyzed in vivo in Drosophila with emphasis on neurogenesis as well as rescue of lethality in wild type and/or $N^{\text {spl }}$ flies. 


\section{Specific Aims:}

1) The UAS constructs of PP2Ac (UAS-mts, UAS-mtsDN and UAS-mtsRNAi) were tested for their ability to elicit neural defects in the eye and bristle.

2) The UAS constructs of PP2Ac were tested for their ability to rescue the neural defects of $N^{s p l}$.

I expect these studies will more precisely describe the mechanisms underlining the dominant behavior of Tik, and further elaborate in the possible antagonistic roles of CK2 and PP2A in eye and bristle development. 


\section{CHAPTER-2}

The dominant effects of Tik during eye and bristle development appear to be a consequence of a 'double hit'. 


\title{
The dominant effects of Tik during eye and bristle development appear to be a consequence of a 'double hit'
}

\author{
Ezgi Kunttas
}

\begin{abstract}
Emerging evidence suggests that CK2 mediates the inhibitory $\mathrm{N}$ signaling via phosphorylation of $\mathrm{E}(\mathrm{spl}) \mathrm{M} 8$. We have previously shown that misexpression of a UAS-Tik encoding a catalytically dead CK2 subunit elicits rough eyes due to excess R8 founders and ectopic and split bristles due to excess SOP's, both indicative of impaired lateral inhibition. Tik harbors two substitutions, $\mathrm{M}^{161} \mathrm{~K}$ and $\mathrm{E}^{165} \mathrm{D}$. The former substitution is thought to predominantly contribute to the dominant behavior of Tik, since it lies in the ATP binding pocket. However, the $E^{165} \mathrm{D}$ mutation might also contribute to the behavior of Tik, given that it resides in a region, which harbors the HEN/HRKL motif, mediates recruitment of the phosphatase PP2A. The possibility thus arises that Tik might be a 'double hit'; the MK substitution neutralizes catalytic function, while the ED substitution perturbs recruitment and/or regulation of PP2A. To test this hypothesis, we generated two variants of CK2 that harbor either of these substitutions, and have analyzed them by a combinatorial approach. As expected, the MK variant displays minimal catalytic activity, whereas that with ED appears closer to the wild type. When tested in vivo, we find that misexpression of either the MK or the ED variant elicits ectopic bristles whose frequency and penetrance closely approximate that seen with Tik. We have also tested for the ability of these variants to suppress the neural defects of $N^{s p l}$. We find that MK and ED suppress the retinal defects of $N^{s p l}$. Given that altered PP2A functions lead to clock and bristle defects, our results raise the possibility that Tik might, in fact, represent a 'double hit'.
\end{abstract}




\section{METHODS}

\section{Bacterial and yeast strains:}

Escherichia coli strain $\mathrm{DH} 5 \alpha$ of was used for all molecular manipulations. The constructs that were created during these studies are schematically represented in Figure 20. All bacterial strains were grown at $37^{\circ} \mathrm{C}$ in Luria Broth (LB, $1 \%$ yeast extract, $2 \%$ Peptone, $0.5 \% \mathrm{NaCl}$ ) containing $150 \mu \mathrm{g} / \mathrm{ml}$ ampicillin.

Multiple strains of yeast $S$. cerevisiae were employed during the course of these studies, which are listed in Table 1. Yeast cells were grown routinely in rich glucose medium (YPD, 1\% yeast extract, $2 \%$ peptone, $2 \%$ dextrose) or rich galactose medium (YPG, 1\% yeast extract, $2 \%$ peptone, $2 \%$ galactose). Selective dropout media, SDD or SDG, were used during yeast transformation, drop out experiments and mating-type two hybrid experiments. The permissive temperature of $29^{\circ} \mathrm{C}$ was used to grow the cells, whereas, $35^{\circ} \mathrm{C}$ and $/$ or $37^{\circ} \mathrm{C}$ were used as nompermissive temperatures depending on the type of the yeast stain. The permissive and non-permissive temperatures are listed in Table 2.

\section{Primers and sequencing:}

The primers that were employed for PCR amplification and/or site-directed mutagenesis (SDM) were synthesized by Integrated DNA Technologies Inc. (IDT), which are listed Table 3 (Appendix A). Sequencing of all PCR-based constructs was performed at the Molecular Genetics Instrumentation Facility (MGIF) at the University of Georgia by using dideoxy chain termination on an Applied Biosystems DNA sequencer 373A. 


\section{Construction of Timekeeper and its variants:}

$d C K 2 \alpha-$ Tik and its variants, $d C K 2 \alpha-\mathrm{MK}$ and $d C K 2 \alpha-\mathrm{ED}$, were created via PCR based SDM. First, the open reading frame (ORF) of wild-type Drosophila CK2 $\alpha(d C K 2 \alpha)$ was amplified by PCR to introduce a BamH1 site and a synthetic Kozak sequence (CAAC) $5^{\prime}$ to the Start (ATG) codon, and a Xho1 site 3' to the Stop (TAG) codon. The PCR product was subcloned into plasmid pBSIIKS(+) (Stratagene), and sequenced. Subsequently, the construct was subject to PCR-based SDM to generate two variants, $M^{161} K$ and $E^{165} D$. The mutants were selected by cleavage of parental DNA by Dpnl digestion. The Dpn-resistant plasmids were transformed into $E$. coli cells via $\mathrm{CaCl}_{2}$ based transformation method and restriction mapped. The constructs were sequenced to verify the presence of only the intended mutations.

\section{Construction of Timekeeper Revertant (TikR):}

$d C K 2 \alpha-T i k R$ was created by using genomic DNA from TikR/Tm3 flies. DNA from male flies was used to amplify CK2 $\alpha$, which is encoded on a single exon. The PCR product was then digested by Mfe-1 (NEB). CK2 $\alpha$ contains a single Mfe1 site, which is lacking in TikR due to the internal deletion. As a result, digestion with Mfe1 allowed 'enrichment' of full-length PCR product encoding TikR. The TikR-ORF was further amplified by using the primers that introduce BamH1 and Kozak sequences 5' to the Start (ATG) codon, and a Xho1 site 3' to the Stop (TAG) codon. The resulting product was subcloned into pBSIIKS(+) and transformed into E. coli. The construct was sequenced to verify the presence of only the intended mutations.

\section{Construction of Timekeeper deletion (TikREK):}

TikREK was created by ligating the first 171 residues from $d C K 2 \alpha$ with the last 166 residues from $d C K 2 \alpha-$ TikR construct. As a result, this construct encodes for a CK2 $\alpha$ variant 
with the internal seven amino acid deletion (234-240) and the $R^{242} E$ substitution, but contains $\mathrm{M}^{161}$ and $\mathrm{E}^{165}$. The $5^{\prime}$ fragment was isolated by digesting WT CK2 $\alpha$ with BamH1 and Dra1 and the 3' fragment was isolated by Dra1 and Xho1. A three-way ligation was conducted to reconstitute the variant. The CK $\alpha-$ TikREK was subcloned into pBSIIKS(+) and transformed into E. coli. The construct was sequenced to verify the presence of only the intended mutations.

\section{Construction of yeast expression plasmids:}

WT $d C K 2 \alpha, d C K 2 \alpha-T i k, d C K 2 \alpha-M K, d C K 2 \alpha-E D, d C K 2 \alpha-T i k R, d C K 2 \alpha-T i k R E K$ and $d C K 2 \alpha-K M$ were subcloned into the BamH1 and Xho1 restriction sites of $\mathrm{pESC}-\mathrm{URA}$ (Stratagene), a high copy Gal-inducible vector for expression of heterologous proteins in S. cerevisiae.

\section{Complement of lethality of yeast lacking endogenous CK2:}

The pESC-URA-WTdCK2 $\alpha$, pESC-URA-dCK2 $\alpha$-Tik, pESC-URA-dCK2 $\alpha-M K, p E S C-$ URA-dCK2 $\alpha-E D, p E S C-U R A-d C K 2-T i k R, ~ p E S C-U R A-d C K 2 \alpha-T i k R E K$ and pESC-URA$\mathrm{dCK} 2 \alpha-\mathrm{KM}$ were transformed into various temperature-sensitive yeast strains using lithiumacetate based protocol (Ausubel et al., 1989). The yeast strains and their corresponding non-permissive temperatures are listed in Table 1 and Table 2. All of the yeast strains used in these studies are generated from the parental strain, RPG41-1a (Padmanabha et al., 1990). RPG41-1a harbors deletions of both the yCKA1 and yCKA2 genes and the lethal effects of these two deletions are rescued by a URA marked plasmid. This URA marked plasmid harbors a wild-type copy of the yCKA2 gene and thus does not exhibit temperature sensitivity. 


\begin{tabular}{|c|c|c|}
\hline $\begin{array}{l}\text { Yeast } \\
\text { Strain }\end{array}$ & Chromosomal Genotype & Plasmid Genotype \\
\hline RPG41-a & MATa cka1-D1::HIS3 cka2-D1::TRP1 & CEN6/ARSH4 URA3 CKA2 \\
\hline YDH8 & MATa cka1-D1::HIS3 cka2-D1::TRP1 & CEN6/ARSH4 LEU2 cka2-8 \\
\hline YEK1 & MATa cka1-D1::HIS3 cka2-D1::TRP1 & $\begin{array}{l}\text { CEN6/ARSH4 LEU2 cka2-8 } \\
2 \text { URA dCK2a-Tik }\end{array}$ \\
\hline YEK2 & MATa cka1-D1::HIS3 cka2-D1::TRP1 & $\begin{array}{l}\text { CEN6/ARSH4 LEU2 cka2-8 } \\
2 \text { URA dCK2 } \alpha-M 161 K\end{array}$ \\
\hline YEK3 & MATa cka1-D1::HIS3 cka2-D1::TRP1 & $\begin{array}{l}\text { CEN6/ARSH4 LEU2 cka2-8 } \\
2 \text { URA dCK2 } \alpha-E 165 D\end{array}$ \\
\hline YEK4 & MATa cka1-D1::HIS3 cka2-D1::TRP1 & 2 URA $d C K 2 \alpha-M 161 K$ \\
\hline YEK5 & MATa cka1-D1::HIS3 cka2-D1::TRP1 & 2 URA $d C K 2 \alpha-E 165 D$ \\
\hline YEK6 & MATa cka1-D1::HIS3 cka2-D1::TRP1 & $\begin{array}{l}\text { CEN6/ARSH4 LEU2 cka2-8 } \\
2 \text { URA dCK2 } \alpha \text {-TikR }\end{array}$ \\
\hline YEK7 & MATa cka1-D1::HIS3 cka2-D1::TRP1 & $\begin{array}{l}\text { CEN6/ARSH4 LEU2 cka2-8 } \\
2 \text { URA dCK2 } \alpha \text {-TiREK }\end{array}$ \\
\hline YEK8 & MATa cka1-D1::HIS3 cka2-D1::TRP1 & $\begin{array}{l}\text { CEN6/ARSH4 LEU2 cka2-8 } \\
2 \text { URA dCK2 } \alpha-K 66 M\end{array}$ \\
\hline YKP6 & MATa cka1-D1::HIS3 cka2-D1::TRP1 & $2 \mu L E U 2 d C K 2 \alpha-W T$ \\
\hline YKP7 & MATa cka1-D1::HIS3 cka2-D1::TRP1 & $2 \mu$ LEU2 dCK2 $\alpha-\mathrm{A} 177 \mathrm{~T}$ \\
\hline YKP10 & MATa cka1-D1::HIS3 cka2-D1::TRP1 & $2 \mu L E U 2 d C K 2 \alpha-G 89 D$ \\
\hline YKP1 & MATa cka1-D1::HIS3 cka2-D1::TRP1 & $\begin{array}{l}\text { CEN6/ARSH4 URA3 CKA2 } \\
2 \mu \text { LEU2 dCK } 2 \alpha-W T\end{array}$ \\
\hline
\end{tabular}

Table 1: S. cerevisiae strains: All strains are essentially derived from RPG41-1a parental yeast strain (Padmanabha et al. (1990)). Yeast strains harbors either single copy centromeric plasmids (CEN6/ARSH4) or multicopy plasmids $(2 \mu)$. The chromosomal and plasmid genotypes of each strain are shown in the table. 


\section{Analysis in yeast rescued by Ts-alleles of dCK2 $\alpha$ :}

YKP6, YKP7 and YKP10 strains carry Leu-marked plasmids, which contain either WT or Ts alleles of dCK2 $\alpha$ (Kuntamalla, unpublished data). YKP6 is a derivative of RPG411a that is rescued by pESC-LEU-dCK2 $\alpha$, whereas YKP7 and YKP10 are rescued by Tsvariants $d C K 2 \alpha A^{177} T$ and $d C K 2 \alpha G^{89} D$. These strains were transformed with pESC-URA$\mathrm{dCK} 2 \alpha-$ Tik, dCK $2 \alpha-\mathrm{MK}$ and dCK $2 \alpha-$ ED constructs and selected on medium lacking Ura and Leu. The ability of these constructs to rescue the lethality was tested on galactose containing medium at permissive $\left(29^{\circ} \mathrm{C}\right)$ and non-permissive $\left(37^{\circ} \mathrm{C}\right)$ temperatures.

\section{Analysis in yeast rescued by Ts-alleles of yCK2 $\alpha$ :}

YDH8 strain carries a Leu-marked plasmid, containing a Ts-allele (ycka2-8) of yCKA2 (Hanna et al., 1995). This strain was individually transformed with the pESC-URAWTdCK2 $\alpha$, pESC-URA-dCK2 $\alpha-T i k, p E S C-U R A-d C K 2 \alpha-M K, p E S C-U R A-d C K 2 \alpha-E D$, pESC-URA-dCK2 $\alpha-T i k R, p E S C-U R A-d C K 2 \alpha-T i k R E K$ and pESC-URA-dCK $2 \alpha-K M$ constructs. The resulting strains were named YEK1-YEK3 and YEK6-YEK8. The ability of these constructs to rescue the lethality was tested on galactose containing medium at permissive $\left(29^{\circ} \mathrm{C}\right)$ and non-permissive $\left(35^{\circ} \mathrm{C}\right)$ temperatures.

\section{Analysis of dropout clones:}

In order to eliminate the possibility that the variants, themselves, were temperature sensitive plasmid drop experiments were conducted (Fig. 17). Selective media was used to select for cells that had randomly dropped the Leu-marked plasmid. The cells, which dropped the Leu-marked plasmids, were essentially 'rescued' by Tik and its variants. To select 'dropouts', cells we re grown in restrictive media to stationary phase for 48 hours 


\begin{tabular}{|l|l|l|l|}
\hline Strain & Allele & $\begin{array}{l}\text { Maximum permissive } \\
\text { temperature }\left({ }^{\circ} \mathbf{C}\right)\end{array}$ & $\begin{array}{l}\text { Minimum restrictive } \\
\text { temperature }\left({ }^{\circ} \mathbf{C}\right)\end{array}$ \\
\hline RPG41-1a & $y C K A 2$ & 38.5 & 40 \\
\hline YKP6 & $d C K 2 \alpha-W T$ & 38.5 & 40 \\
\hline YKP7 & $d C K 2 \alpha-A 177 T$ & 32 & 37 \\
\hline YKP10 & $d C K 2 \alpha-G 89 D$ & 35 & 37 \\
\hline YDH8 & ycka2-8 & 33 & 35 \\
\hline
\end{tabular}

Table 2: Temperature sensitivity of Ts strains: The temperature sensitive strains that were employed during this study are listed in the table. The maximum permissive temperature is the highest temperature, which allows normal colony formation on YPG/YPD plates. The minimum restrictive temperature is the lowest temperature, which prevents observable growth on YPG/YPD plates. 
A
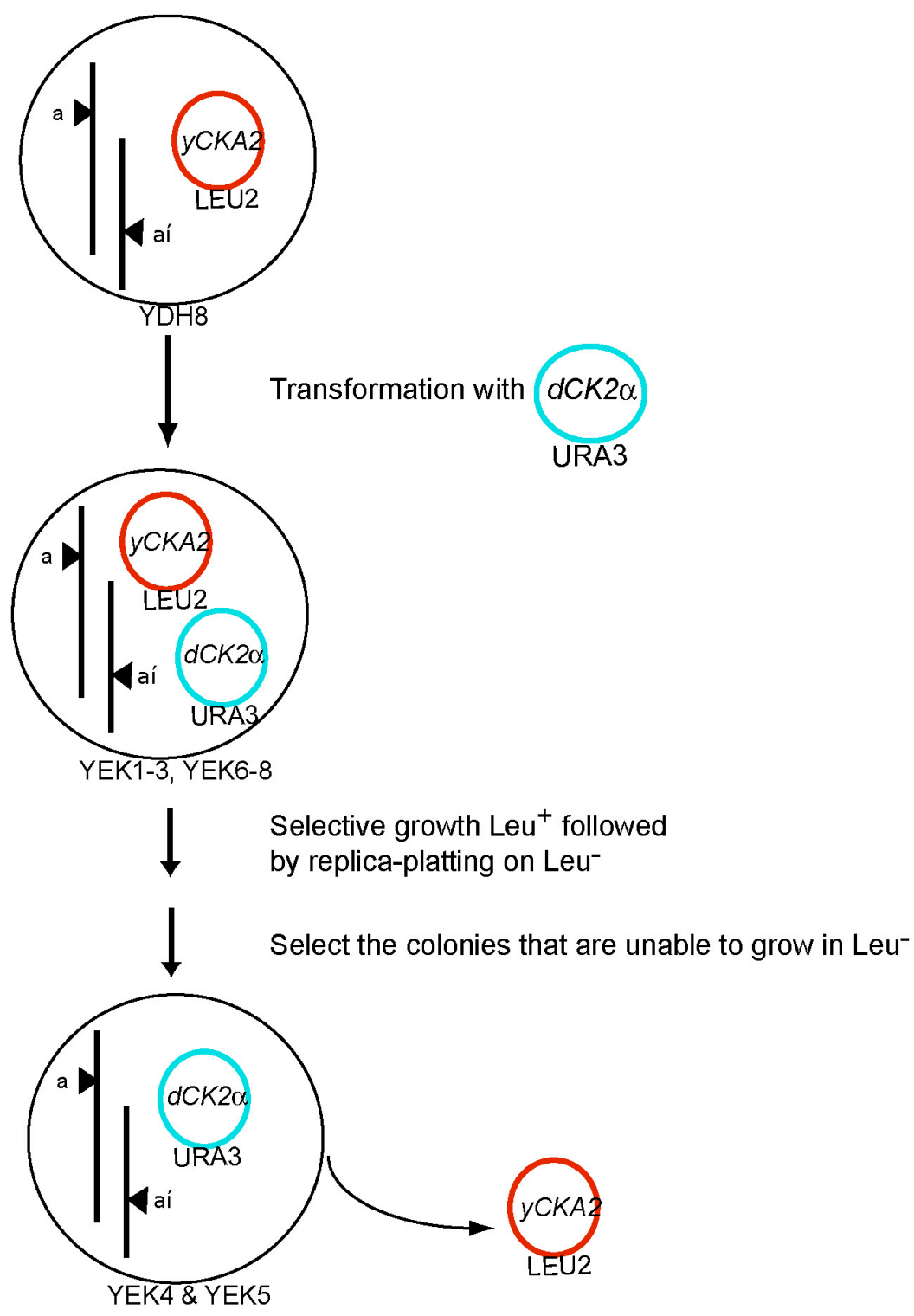

dCK $2 \alpha$ pESC-URA-CK $2 \alpha-W T$, pESC-URA-CK $2 \alpha-T i k$, pESC-URA-CK $2 \alpha-M K$, pESC-URA-CK $2 \alpha-E D$, pESC-URA-CK $2 \alpha-T i k R$, pESC-URA-CK $2 \alpha-T i k R E K$, pESC-URA-CK $2 \alpha-K M$

Figure 17: Analysis of dropout clones: Plasmid drop experiments were conducted to eliminate the possibility that the $d C K 2 \alpha$-variants, themselves, were temperature sensitive. Selective media was used to choose for cells that had randomly dropped the Leu-marked plasmid and are thus 'rescued' by Tik and its variants. 
at $29^{\circ} \mathrm{C}$, the permissive temperature. Subsequently, $200 \mu \mathrm{l}$ of the culture was transferred to fresh media and grown to saturation. This procedure was repeated for a total of 4 rounds. At the end of the $4^{\text {th }}$ round, $250 \mu$ l cells were plated on a Ade, Lys and Leu containing media. Individual clones were tested for the absence of the Leu-marked plasmid by replica-plating the cells onto $\mathrm{CDG}(\mathrm{AKL})^{+}$and $\mathrm{CDG}(\mathrm{AK})^{+}$media. The cells that dropped the Leu-marked plasmid were unable to grow on the media lacking Leu. 250 clones were analyzed for each variant. The dropout clones for dCK $2 \alpha-M K$ and dCK2 $\alpha-E D$ were obtained, which were named YEK4 and YEK5. These clones were analyzed for rescue the growth arrest of YDH8 at $35^{\circ} \mathrm{C}$ and $37^{\circ} \mathrm{C}$ (NPT).

\section{Determination of mean generation time and terminal phenotypes:}

The growth rates of each strain were measured by calculating the mean generation times. Yeast strains were grown in YPG at $29^{\circ} \mathrm{C}$ for $24 \mathrm{hrs}$ and diluted into $50 \mathrm{ml}$ of fresh YPG at a starting density of $5 \times 10^{5}$ cells $/ \mathrm{ml}$. Cultures were grown at $29^{\circ} \mathrm{C}$ and aliquots of the cultures were collected every two hours over a 16 -hour period. The cells were fixed by the addition of formaldehyde to a final concentration of $3.7 \%$. A hemacytometer was used to determine the number of cells for each sample. The mean-generation time was calculated from the slope of exponential growth rates of cultures.

Yeast strains were grown in YPG at $29^{\circ} \mathrm{C}$ for $24 \mathrm{hrs}$ and diluted into $50 \mathrm{ml}$ of fresh YPG at a starting density of $5 \times 10^{5}$ cells $/ \mathrm{ml} .25 \mathrm{ml}$ of these cultures were shifted into the non-permissive $\left(35^{\circ} \mathrm{C}\right)$ and grown for additional 16 hours. Samples were collected and fixed by the addition of formaldehyde to a final concentration of $3.7 \%$ and photographed under DIC using an Olympus AX70 confocal microscope at a magnification of 400X.

\section{Expression and stability of CK2 in yeast:}

The strains encoding for dCK2 variants were grown in YPG overnight at $29^{\circ} \mathrm{C}$ and 
equal number cells $\left(2 \times 10^{7}\right)$ from each strain were harvested. The cells were washed in $1 \times$ Phosphate Buffered Saline (PBS) and prepared by the addition of Laemmli buffer (60mM Tris-Hcl, pH 6.8, 10\% glycerol, 2\% sodium dodecyl sulfate (SDS), 1\% 2-mercaptoethanol and $0.002 \%$ bromophenol blue) followed by boiling for 10 min (Laemmli, 1970). Cell extracts were separated by two SDS-polyacrylamide gel electrophoresis (SDS-PAGE) gels. The first gel was stained with Coomassie Blue $(0.05 \%)$ in $5 \%$ methanol and $10 \%$ acetic acid overnight. The gel was destained the next day in 5\% methanol and $10 \%$ acetic acid. The second gel was transferred into a nitrocellulose membrane (Osmonics, Inc) electrophoretically (Bidwai et al., 1992; Towbin et al., 1979). The membrane was washed in TBST buffer (10 mM Tris pH 8.0, $150 \mathrm{mM} \mathrm{NaCl}, 1 \%$ BSA, 0.05\% Tween 20, 0.02\% NaN3) for 3 hours. Then the membrane was incubated with the polyclonal (rabbit) antibody raised against Drosophila CK2 holoenzyme (Dahmus et al., 1984) with TBST at $4^{0} \mathrm{C}$ overnight. The next day, the membrane was washed again with TBST and incubated with the secondary antibody (affinity purified goat-anti-rabbit lgG) coupled to alkaline phosphatase (BioRad). After washing the membrane with AP Buffer (100 mM Tris-Cl, pH 9.5, $100 \mathrm{mM}$ $\mathrm{NaCl}, 5 \mathrm{mM} \mathrm{MgCl}$ ) the immunoblot was developed by adding nitroblue tetrazolium (NBT, $460 \mu \mathrm{g} / \mathrm{ml}$ ) and 5-bromo-4-chloro-3-indolyl phosphate (BCIP, $16.5 \mu \mathrm{g} / \mathrm{ml}$ ). Reactions were terminated by the addition of EDTA to a final concentration of $50 \mathrm{mM}$. The gel and the blot were scanned and processed by using Adobe Illustrator and Photoshop.

\section{Protein-protein interactions using yeast 2 Hybrid (Y2H) analysis:}

Interactions between $d C K 2 \alpha$ and its variants with $d C K 2 \beta$ were studied by using a mating type two-hybrid system (Fig. 18). In this system, proteins are expressed as fusions with the DNA-binding $(\mathrm{DB})$ domain in one mating type and the activation-domain $(A D)$ in the 

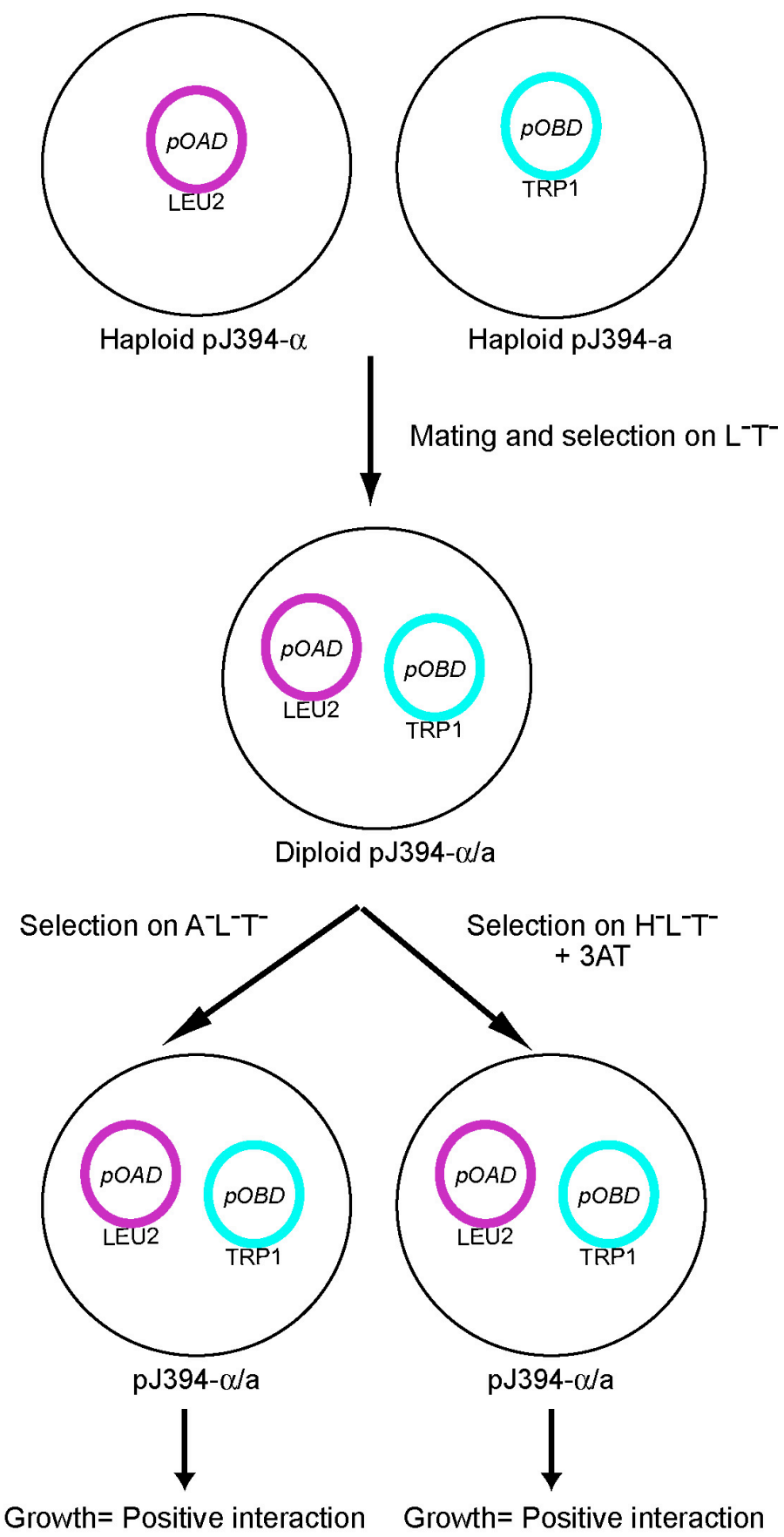

Figure 18: Mating type two-hybrid assay: Interactions between $d C K 2 \alpha$ and its variants with $d C K 2 \beta$ were studied by using a mating type two-hybrid system. $d C K 2 \alpha$-variants are expressed as activation-domain $(A D)$ and $d C K 2 \beta$ as $D N A$ binding $(D B)$ domain. The $A D$ and $B D$ plasmids were transformed into different mating types of pj69-4 strain and mated. The resulting diploids were selected on selective media and tested for interactions. Growth on selective media indicates a positive interaction between $A D$ and $B D$ constructs. 
other mating type. $d C K 2 \alpha$ and its variants CK2 $\alpha-$ Tik, CK2 $\alpha-\mathrm{MK}, \mathrm{CK} 2 \alpha-\mathrm{ED}, \mathrm{CK} 2-\mathrm{TikR}$ and CK2 $\alpha$-TikREK were subcloned into EcoR1 and Sal1 sites of the AD plasmid, pOAD, which is marked with the selectable marker LEU2. The resulting construct then was transformed into

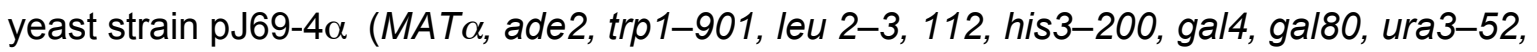
GAL7-lacZ::met1, GAL2-ADE2::ADE2, GAL1-HIS3::LYS2). dCK2 $\beta$ was subcloned into EcoR1 and Pst1 sites of the DB plasmid, pOBD, which is marked with the selectable marker TRP1. The resulting construct was transformed into yeast strain pJ694-a (MATa, ade2, trp1-901, leu 2-3, 112 his3-200, gal4, gal80, ura3-52, GAL7-lacZ::met1, GAL2ADE2::ADE2, GAL1-HIS3::LYS2). Mating between pJ69-4 $\alpha$ and pJ69-4a was achieved by mixing $250 \mu \mathrm{l}$ of each culture in YPD at $29^{\circ} \mathrm{C}$ overnight. The diploid cells were plated on a Trp and Leu lacking media, and individual colonies were selected for further analysis. Cells were plated on two different selective media for the analysis of two-hybrid interactions, the first one lacking His, Trp, and Leu and the second one lacking Ade, Trp, and Leu. Plates were incubated at $29^{\circ} \mathrm{C}$ for $3-5$ days. Growth was an indication of positive interactions between the $\mathrm{pOAD}$ and $\mathrm{pOBD}$ constructs. In order to eliminate leaky expression of basal levels of HIS3 gene product, 1mM 3-amino-triazole (3AT) to the plates.

PJ69-4 strain can also be used to measure automated measurement of the $\beta$ galactosidase activity since it contains a Gal7 promoter-regulated lacZ gene. Therefore, strength of the interactions were determined by measuring the LacZ activity, for which ONitrophenyla -D-Galactopyranoside (ONPG) was used as a substrate. The cells were grown overnight in minimal synthetic defined (SD) base with galactose plus raffinose (Gal/Raf). Cells were grown in YPG until the Optical Density at $600 \mathrm{~nm}$ (OD600) was reached around 0.7-0.8. Cells were then harvested and washed twice with $1 \times Z$ buffer $\left(60 \mathrm{mM} \mathrm{Na}_{2} \mathrm{HPO}_{4}, 10\right.$ $\mathrm{mM} \mathrm{KCl}, 1 \mathrm{mM} \mathrm{MgSO}_{4}$ ). 50mM-2-mercaptoethanol in $\mathrm{Z}$ buffer was added and cells were frozen by liquid nitrogen for $30 \mathrm{sec}$. reheated at $37 \mathrm{C}$ water bath, which was repeated three 
times. Subsequently, ONPG in Z buffer was added and cells were incubated at 30C for $\sim 15$ minutes. At least three independent transformants were tested for induction of LacZ using this assay (Miller, 1972). LacZ activity was calculated by using the formula $1000 x O D 420 /(T x V x O D 600)$, where $T$ is in minutes and $V$ is the concentration factor of the assay.

\section{Molecular modeling:}

Swiss-pdb viewer is used to generate the molecular models used in this study. Molecular models for $d C K 2 \alpha$ and its variants were generated via $1 \mathrm{jwh}$ pdb file, which is available through the pdb data bank (www.rcsb.org) (Niefind et al., 2001). The mutational analysis was also utilized to visualize the possible alterations of the structure.

\section{Transgenic flies:}

The GAL4-UAS system was employed for in vivo analysis in Drosophila with the $d C K 2 \alpha$ variants, $d C K 2 \alpha-M K$ and $d C K 2 \alpha-E D$. Binary Gal-UAS system was developed by Norbert Perrimon and Andrea Brand in 1993 (Fig. 19) (Duffy, 2002). Since then, this system has been used extensively to control the expression of a transgene. It takes advantage of the fact that yeast protein GAL4 is silent and doesn't activate any of the Drosophila genes due to the absence of GAL4 binding sequences, Upstream Activating Sequences (UAS) (Duffy, 2002). Therefore, transgenes can be engineered with an UAS element that allows for GAL4 binding. In order to achieve this, the transgene and the driver are maintained in separate parental lines and the expression of a transgene is only achieved when flies harboring the UAS-transgene are mated with the flies expressing the GAL4 element. The collection of GAL4 fly stocks has been generated by random insertions of an enhancer-less GAL4 cassette. These randomized insertions allowed for the expression of GAL4 in a developmental stage/tissue specific manner (Brand and Perrimon, 1993; Klueg et al., 2002). 
The cDNAs of $d C K 2 \alpha-\mathrm{MK}$ and $d C K 2 \alpha-E D$ were isolated and subcloned into the Bglll and Xhol sites of the pUAST vector, which carries UAS elements upstream of the transgene. Germline insertions were accomplished by injections of the constructs into $w^{1118}$ embryos were conducted by Best Gene Inc. The presence of the transgene was selected via the presence of the mini-white gene $\left(w^{+}\right)$, which elicits a red eye color. Multiple transgenic lines were obtained for each variant and the chromosomal insertions were mapped by multiple crosses to the balanced stocks harboring dominant markers. The schematic of crosses conducted during mapping can be found in Figure 27.

\section{Overexpression and phenotypic analysis in Drosophila:}

Flies were raised at $24^{\circ} \mathrm{C}$ or $29^{\circ} \mathrm{C}$ on a yeast extract and glucose containing media. The Gal4 lines used in these studies were obtained from the Bloomington Stock Center at Indiana University. The Gal4 drivers used in these studies are, G455.2 and scaGal4, eyGal4, and act5Gal4 (Giebel and Campos-ortega, 1997). The expression domains of the drivers are shown in Figure 16. The ability of UAS-CK2 $\alpha, U A S-C K 2 \alpha-T i k, U A S-C K 2 \alpha-M K$, and UAS-CK2 $\alpha-E D$ to elicit neural defects in the eye or in the bristle lineage was tested. UAS-CK $2 \alpha$ line has been previously created in our lab, UAS-CK $2 \alpha-T i k$ line was a gift from Ravi Allada, Northwestern University, and UAS-CK2 $\alpha-M K$ and UAS-CK $2 \alpha-E D$ lines were created during the course of this study. Light microscopy was employed for the imaging of the phenotypes. Scanning electron microscopy (SEM) analysis was used for the detailed eye images. For SEM analysis, flies were passed thorough series of dilutions of alcohol series (25\%-50\%-75\%-absolute), and finally passed through Hexamethyldisalizane. The samples were sputter coated, and examined with a JEOL-6400 or Hitachi S-4700 scanning electron microscope. The Polaroid pictures were scanned and all images were processed 



Figure 19: The Gal4-UAS system in Drosophila: The GAL4-UAS bipartite system is widely used in the fruit fly for the targeted expression of transgenes. This system was employed for in vivo analysis of the $d C K 2 \alpha-\mathrm{MK}$ and $d C K 2 \alpha-E D$ variants. In this two-part approach, the transgene and the driver are maintained in separate parental lines and the expression is only achieved when flies harboring the UAS-transgene are mated with the flies expressing the GAL4. 
by Adobe Photoshop and Adobe Illustrator.

\section{Rescue of $\boldsymbol{N}^{s p l}$ phenotype:}

Gal4-drivers were combined with the $N^{\text {spl }}$ allele by conducting standard protocols. Since $N^{s p l}$ is recessive and X-linked only the male progeny were scored for the analysis. Light microscopy and SEM analysis were employed as described above. Immunostaing of the eye imaginal discs were employed for further analysis of cell fates. Eye imaginal discs were isolated from late third instar larvae, and processed as described (Kavler et al., 1999). Eye discs were fixed in $4 \%$ paraformaldehyde in $1 x$ phosphate buffered saline (PBS) and washed three times with PBS containing $0.1 \%$ Triton X-100 (PBS-TX). The primary antibody stain was conducted overnight at $4^{\circ} \mathrm{C}$ in anti-Ato and anti-sense antibodies at a 1:800 and 1:1000 dilutions respectively. After washing three times, the discs were incubated with the secondary antibody for three hours. The eye discs were mounted in Vectashield and images were acquired with an Olympus XL confocal Microscope. The images were processed using Adobe Photoshop and Adobe Illustrator. 


\section{RESULTS AND DISCUSSION}

\section{Ability of CK2 variants to complement lethality of yeast lacking endogenous CK2:}

As mentioned above (see Introduction), a dominant allele of CK2 $\alpha$, Tik, harbors two amino acid substitutions, $M^{161} \mathrm{~K}$ and $E^{165} \mathrm{D}$. The dominant behavior of Tik is thought to derive from the former substitution since it lies in the ATP binding pocket (Fig.10). It has been proposed that the introduction of a positively charged Lys into a relatively hydrophobic ATP-binding pocket is likely to disrupt nucleotide binding. In contrast, the latter substitution has been thought to be 'silent' and not contribute to the dominant behavior of Tik. This proposal underlies the assumption that replacement of $\mathrm{Glu}^{165}$ with another acidic residue (Asp) is unlikely to significantly alter the electrostatic properties and thus function. This possibility was underscored with the finding that $\mathrm{Glu}^{165}$ is located in a region of CK2 $\alpha$ that is positioned in the hinge region between the two catalytic domains and points away from the active site. However, the $E^{165} \mathrm{D}$ mutation resides within the 'putative' PP2A binding site, which is mediated via the HEN/HRKL motif (Fig. 10). As mentioned before, this motif is shared between CK2 $\alpha$ and SV40 small-t antigen only and might serve to regulate the activities of either enzyme. We hypothesized that Tik might thus represent a 'double hit', whereby disrupted ATP-binding as well as loss of regulatory interactions due to missing/altered PP2A binding underlie its dominant behavior. To test this hypothesis, two variants of CK2 $\alpha$ were generated that contain these individual substitutions. These are, $C K 2 \alpha-M^{161} K$ and $C K 2 \alpha-E^{165} D$. Henceforth, I refer to these two variants as CK2 $\alpha-M K$ and CK2 $\alpha$-ED, respectively. The schematic of all the variants can be found in Figure 20. TikR harbors the two original mutations seen in Tik $\left(\mathrm{M}^{161} \mathrm{~K}\right.$ and $\left.\mathrm{E}^{165} \mathrm{D}\right)$, and, in addition, deletes seven amino acids (234-240) and substitutes $\operatorname{Arg}^{242} \mathrm{Glu}$. It has been proposed that TikR displays folding problems due to the internal deletion and is, therefore, unable to bind to CK2 $\beta$. In addition, since TikR harbors the $\mathrm{M}^{161} \mathrm{~K}$ mutation, it has been assumed that this 

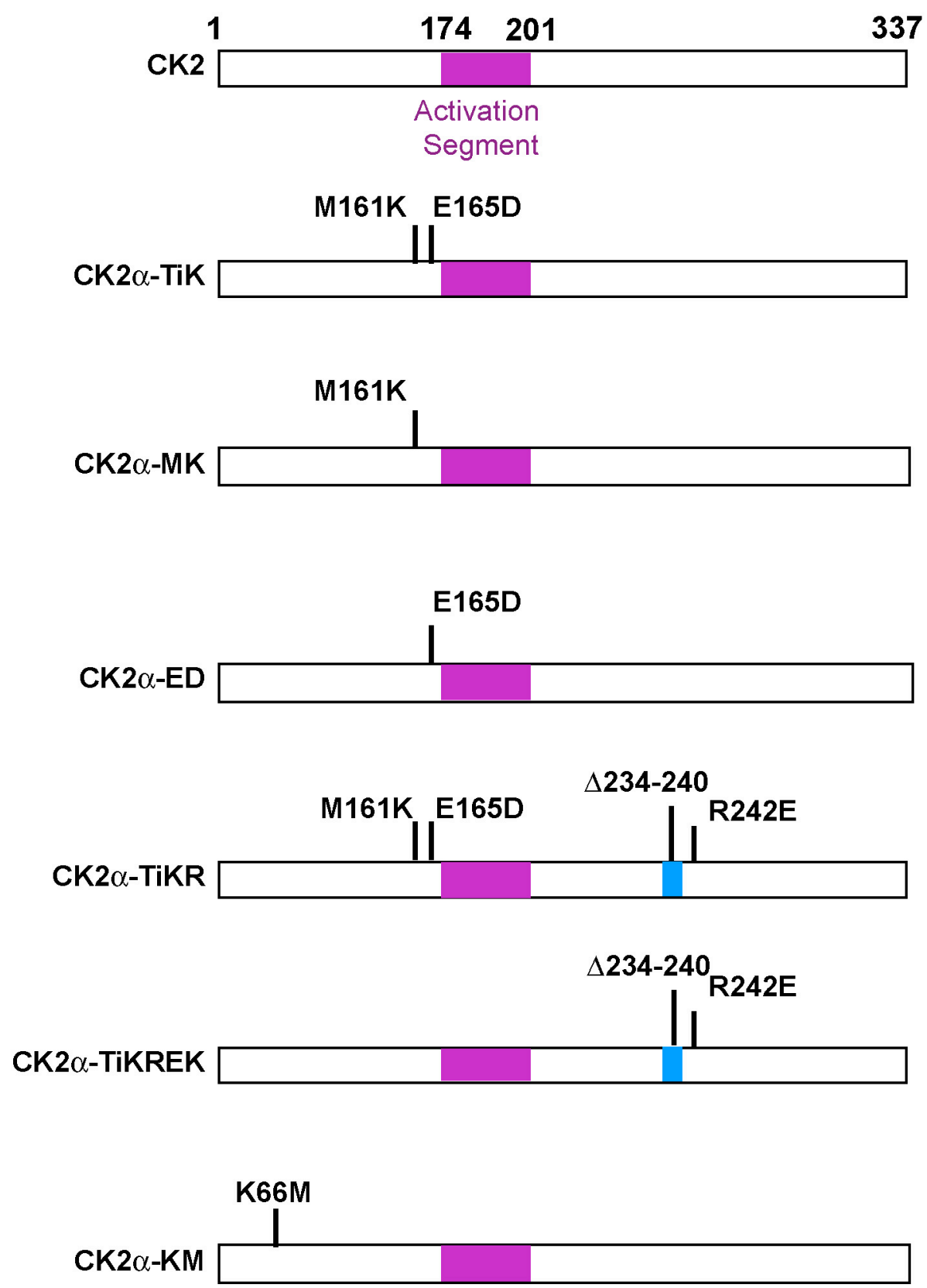

Figure 20: The schematic of constructs: The constructs that were employed during the course of this study are shown in the figure. The relative positions of the amino acid substitutions and/or deletions (blue color) found in all variants are shown. 
variant is also catalytically compromised. In order to test this hypothesis, a variant of TikR was generated, which harbors the seven amino acid deletion plus the $\mathrm{R}^{242} E$ substitution, but harbors Met and Glu at positions 161 and 165, respectively. It was predicted that this variant might be catalytically active, but deficient for CK2 $\beta$ binding.

Lastly, a CK2 $\alpha-K^{66} \mathrm{M}$ variant was employed to compare the catalytic activity of Tik, MK and TikR. This CK2 $\alpha-K^{66} \mathrm{M}$ allele has previously been shown to be deficient for phospho-transferase activity, which renders the enzyme catalytically dead. $\mathrm{K}^{66} \mathrm{M}$ substitutions resides right in the ATP binding loop and is essential for enzymatic activity. On the other hand, $\mathrm{M}^{161} \mathrm{~K}$ is predicted not to affect the phospho-transferase activity, but disrupts ATP binding (Fig. 21). Therefore, it was hypothesized that Tik and TikR should act like $\mathrm{CK} 2 \alpha-\mathrm{K}^{66} \mathrm{M}$ if they are truly catalytically dead.

As mentioned earlier, deletion of both yCK $2 \alpha$ subunits is lethal in yeast (Padmanabha et al., 1990) and, this lethality can be rescued by expression of a cDNA encoding Drosophila CK2 $\alpha$ (Bidwai et al., 1992). In addition, it has been shown that CK2 $\alpha-$ $\mathrm{K}^{66} \mathrm{M}$ cannot elicit any rescue. These findings demonstrates that complementation of lethality is an appropriate means to compare the relative activities of Tik, TikR and their variants. All of these variants (see Methods) were expressed in yeast using a Gal-inducible (high copy) yeast expression vector pESC-URA. Multiple yeast strains were employed for these studies.

\section{Analysis in yeast rescued by Ts-alleles of dCK2 $\alpha$ :}

Our laboratory has recently identified several Ts-variants of $d C K 2 \alpha$ (Kuntamalla and Bidwai, unpublished data). These variants rescue the lethality of yeast lacking yCK2 subunits, but in a temperature-sensitive manner. I reasoned that these Ts-strains would 


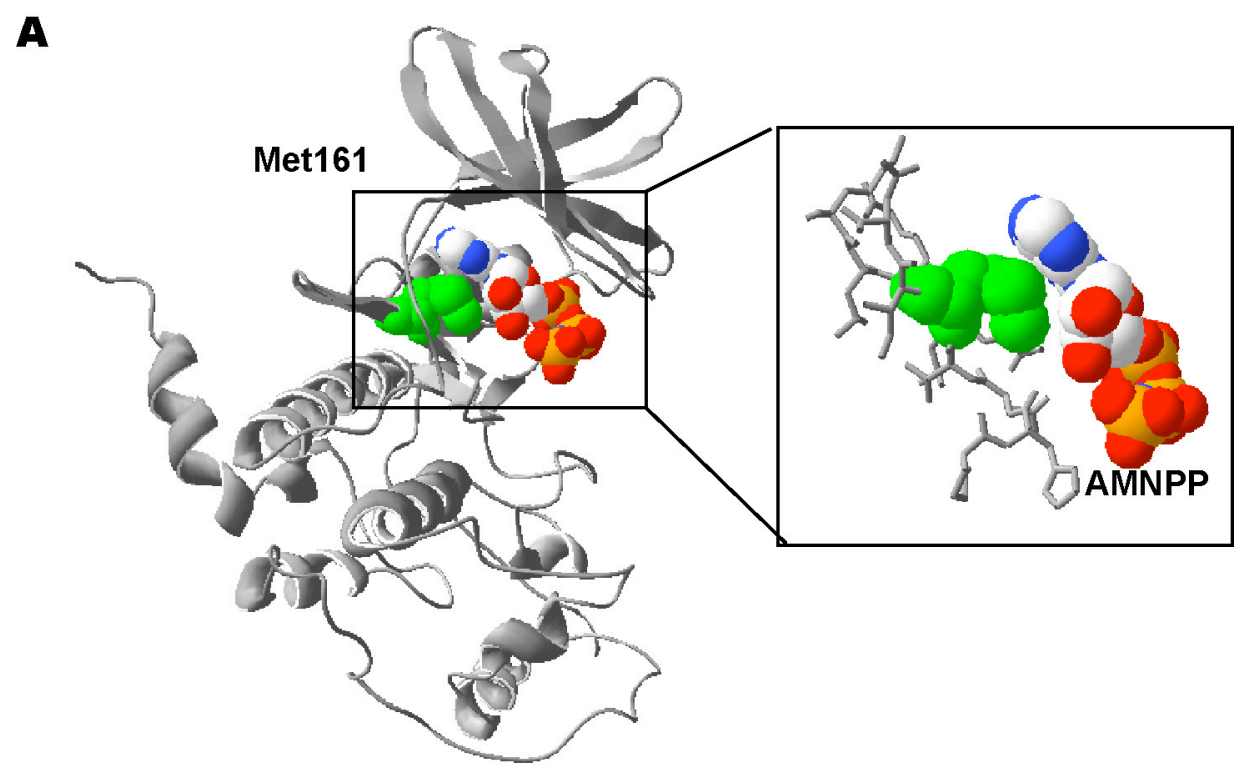

B
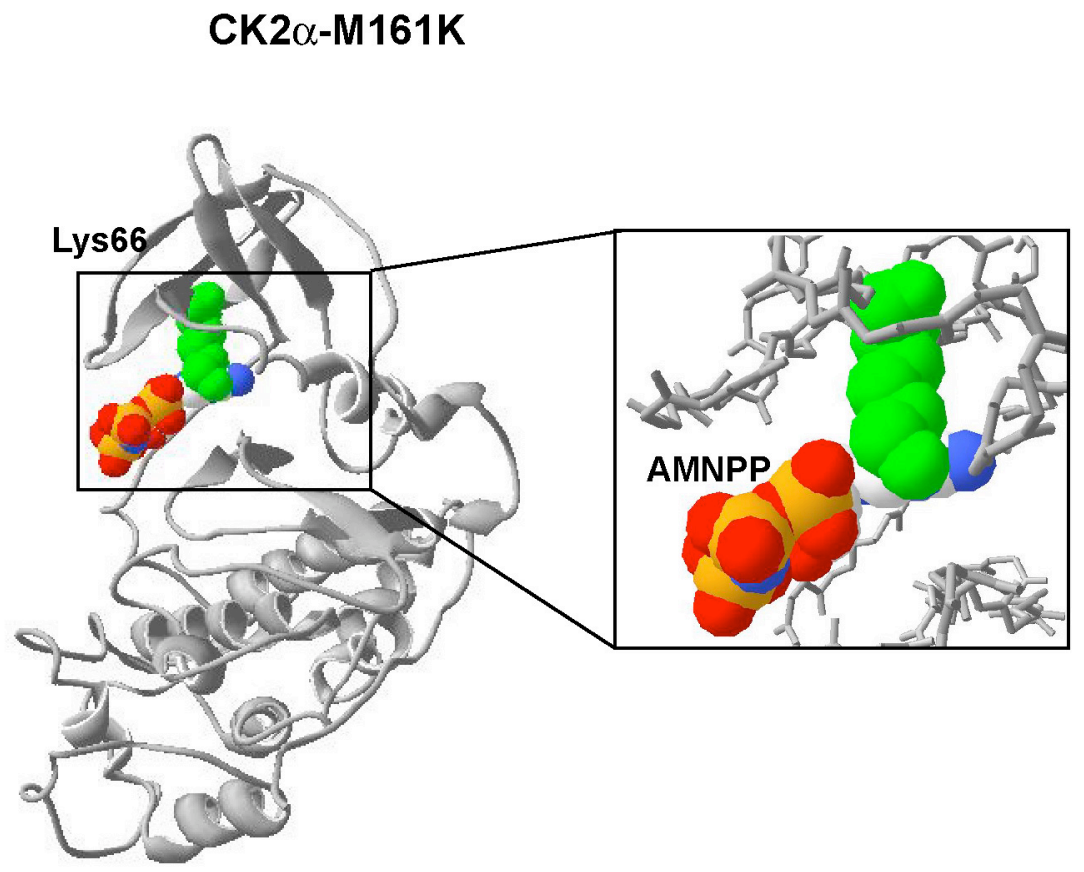

CK2 $\alpha-K 66 M$

Figure 21: Structural comparison between $M^{161} K$ and $K^{66} M$ substitutions: A) The position of $\mathrm{M}^{161} \mathrm{~K}$ substitution relative to the ATP binding. This substitution is predicted to disrupt the ATP binding. B) The position of $\mathrm{K}^{66} \mathrm{M}$ substitution relative to the ATP binding. This substitution has previously shown to render the enzyme deficient for phospho-transferase activity, which is essential for activity of the enzyme. 
enable a rapid assessment of the relative activities of Tik and its two variants, CK2 $\alpha$ MK and CK2 $\alpha$-ED. The Ts yeast strains used in these studies are YKP7 and YKP10, whose permissive temperature is $29^{\circ} \mathrm{C}$ and non-permissive temperature is $37^{\circ} \mathrm{C}$. As a control, I employed the yeast strain YKP6, which is rescued by WT-dCK2 $\alpha$, and does not exhibit any temperature sensitive effects on viability.

These strains were transformed with plasmids expressing CK2 $\alpha-\mathrm{Tik}, \mathrm{CK} 2 \alpha-\mathrm{MK}$ and CK2 $\alpha-E D$, and cells were selected for the presence of both plasmids on selective media. Cells harboring two plasmids were grown overnight to saturation, and equal numbers were spotted to YPG plates that were incubated at $29^{\circ} \mathrm{C}$ (the PT) and at $37^{\circ} \mathrm{C}$ (the NPT) for $2-3$ days. As shown in Figure 22, the non-Ts strain YKP6 (the control) grows efficiently at both temperatures. In contrast, the two Ts-strains, YKP7 or YKP10 grow efficiently at $29^{\circ} \mathrm{C}$, but exhibit complete growth arrest at the higher temperature. The low level/sporadic growth of YKP10 at $37^{\circ} \mathrm{C}$ has been previously observed (Kuntamalla and Bidwai, unpublished), and might reflect either a low frequency of reversion or a 'leaky' NPT. This 'leaky' behavior of Ts alleles is a frequent observation. We next assessed the ability of Tik, CK2 $\alpha-\mathrm{MK}$ and CK2 $\alpha-$ ED to rescue the growth arrest of $Y K P 7$ and $Y K P 10$ at $37^{\circ} \mathrm{C}$. As expected, these strains grew efficiently at the permissive temperature, reflecting full activity of $d C K 2 \alpha-T$ s alleles. Consistent with the behavior of Tik in Drosophila, this variant did not rescue the growth arrest of $\mathrm{YKP} 7$ or $\mathrm{YKP} 10$ at $37^{\circ} \mathrm{C}$, indicating that it is non-functional. Similarly, CK2 $\alpha-\mathrm{MK}$ was also found to be non-functional in this assay. However, it is important to note that expression of Tik or CK2 $\alpha-\mathrm{MK}$ in yeast YKP6 (harboring wild type dCK2 $\alpha$ ) elicited lower growth at the NPT, but not at the PT. While the reason(s) for this are currently unknown, it is reasonable to speculate that the wild type protein, might itself, be close to or at the threshold for loss of activity, and that Tik or CK2 $\alpha-\mathrm{MK}$ might be acting in a dominantnegative manner. This behavior would not be expected to manifest at the PT, where dCK2 $\alpha$ 
is fully functional. On the other hand, CK2 $\alpha$-ED appeared to rescue the temperaturesensitivity of either YKP7 or YKP10, raising the possibility that this variant is not compromised for catalytic activity. Thus these studies suggest that CK2 $\alpha$-Tik and CK2 $\alpha-\mathrm{MK}$ are non-functional, whereas CK2 $\alpha-E D$ is not compromised.

\section{Analysis in yeast rescued by Ts-alleles of yCK2 $\alpha$ :}

As previously shown (see above), cells co-expressing dCK2 $\alpha+$ CK $2 \alpha-T i k$, or $\mathrm{dCK} 2 \alpha+\mathrm{CK} 2 \alpha-\mathrm{MK}$ exhibited a lower extent of growth as compared to yeast expressing only $\mathrm{dCK} 2 \alpha$. These findings led to the possibility that Tik or CK2 $\alpha-\mathrm{MK}$ might act in a dominant manner and preclude a precise assessment of their in vivo activities. To obviate this possibility, analysis was conducted in a strain harboring a Ts allele (ycka2-8) of yCK2,

YDH8. Most importantly, it has been previously shown that $d C K 2 \alpha-K^{66} \mathrm{M}$, which is catalytically dead, does not affect in a dominant manner the behavior of the ycka2-8 allele. Thus YDH8 would bypass any such confounding issue. In addition, the NPT for yeast strain $\mathrm{YDH} 8$ is $35^{\circ} \mathrm{C}$, a temperature that is lower than that for $\mathrm{YKP} 7$ and $\mathrm{YKP} 10\left(37^{\circ} \mathrm{C}\right)$. Moreover, this lower temperature would avoid any heat shock effects, an important consideration given that Hsp's are targets of CK2 in vivo (Pinna, 2002). As a control, I employed the yeast strain RPG41-1a, which is rescued by WT-yCK2 $\alpha$, and does not exhibit any temperature sensitivity. An additionally control was $\mathrm{CK} 2 \alpha-\mathrm{KM}$, which was previously shown to not rescue lethality. Cells were grown at the PT $\left(29^{\circ} \mathrm{C}\right)$ and equal number of cells was spotted on YPG plates. The plates were incubated at $29^{\circ} \mathrm{C}$ (the PT) and at $35^{\circ} \mathrm{C}, 37^{\circ} \mathrm{C}$ (the NPT) for $2-3$ days.

We first assessed the ability of CK2 $\alpha$-Tik, CK2 $\alpha-M K$ and CK2 $\alpha$-ED to rescue the growth arrest of YDH8 at $35^{\circ} \mathrm{C}$ (Fig. 23). As expected, the strain grew efficiently at the 
A

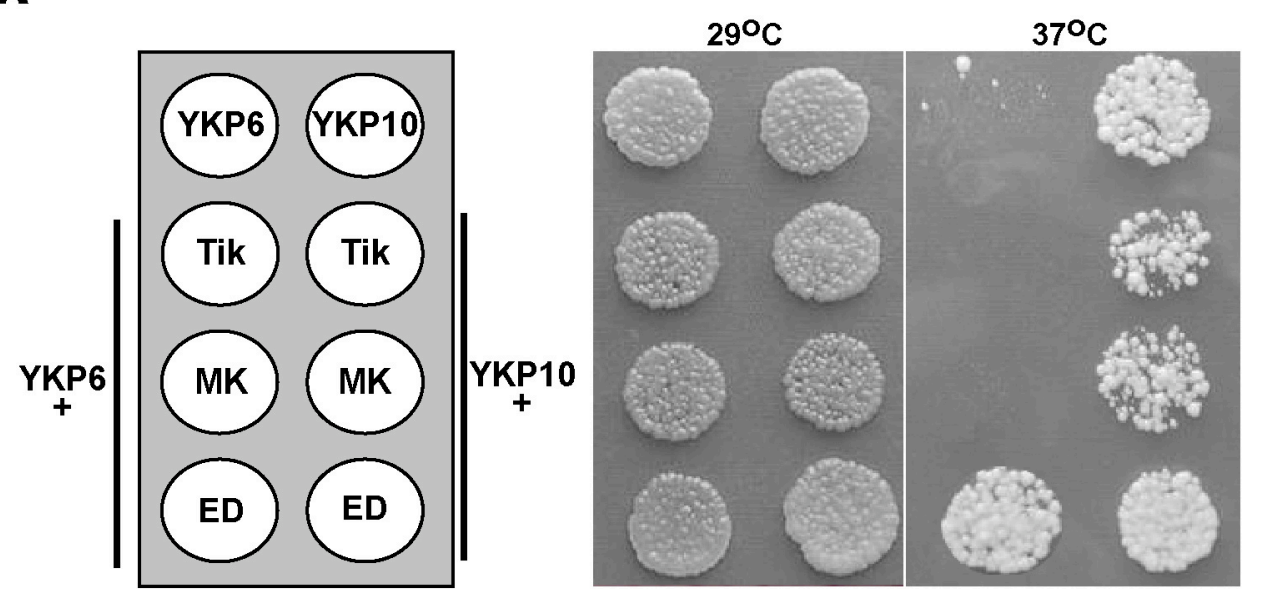

B
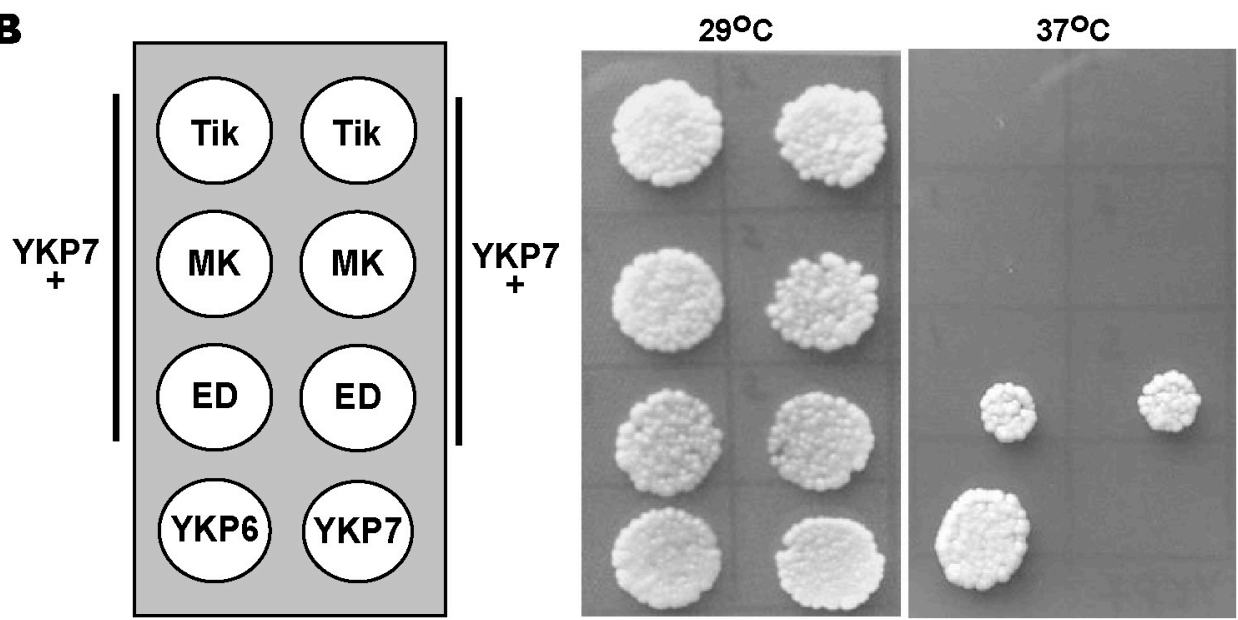

Figure 22: Analysis in yeast rescued by Ts alleles of yCK2 $\alpha$ : Tik and its two variants, CK2 $\alpha-M K$ and CK2 $\alpha-E D$, were tested for their ability to rescue the lethality of yeast which is null for CK2. The Ts yeast strains used in these studies are YKP7 and YKP10, whose permissive temperature is $29^{\circ} \mathrm{C}$ and non-permissive temperature is $37^{\circ} \mathrm{C}$. The non-Ts strain YKP6 (the control) grows efficiently at both temperatures. In contrast, the two Ts-strains, YKP7 or YKP10 grow efficiently at $29^{\circ} \mathrm{C}$, but exhibit complete growth arrest at the higher temperature. CK2 $\alpha-T i k, C K 2 \alpha-M K$ and $C K 2 \alpha-E D$ were tested to rescue the growth arrest of YKP7 and YKP10 at $37^{\circ} \mathrm{C}$. It is found that Tik and CK2 $\alpha-M K$ could not rescue the lethality, unlike CK2 $\alpha-E D$, which rescued. Thus these studies suggest that CK2 $\alpha-$ Tik and CK2 $\alpha-M K$ are non-functional, whereas CK2 $\alpha-E D$ is not compromised. 
permissive temperature, reflecting full activity of yCK2 $\alpha-$ Ts allele. Consistent with the behavior of Tik in Drosophila and yeast, this variant did not fully rescue the growth arrest of YDH8 at $35^{\circ} \mathrm{C}$, indicating that it is non-functional. Similarly, CK2 $\alpha-M K$ was also found to not be fully functional in this assay. It is important to note that at the NPT, cells expressing Tik or CK2 $\alpha-\mathrm{MK}$ displayed marginal, but discernable, growth, a characteristic not associated with $\mathrm{YDH} 8$ on its own. Since $\mathrm{YDH} 8$ displays a complete growth arrest at $35^{\circ} \mathrm{C}$, it is reasonable to suggest that Tik and CK2 $\alpha-\mathrm{MK}$ might have basal activity, one that is permissive for slow growth. Alternatively, it is possible that Tik and CK2 $\alpha-\mathrm{MK}$ represent variants that are, by themselves, Ts for activity. This possibility is supported by the finding that cells co-expressing yCKA2+Tik or yCKA2+CK2 $\alpha-M K$ exhibit low levels of growth at $35^{\circ} \mathrm{C}$, but not at $37^{\circ} \mathrm{C}$. In contrast, CK2 $\alpha$-ED acted in an analogous manner to the WT.

Next, we tested for the ability of CK2 $\alpha-$ TikR and CK2 $\alpha$-TikREK to rescue the growth arrest of YDH8 at $35^{\circ} \mathrm{C}$, but found that this was not the case. The inability of CK2 $\alpha$-TikR to rescue was expected. On the other hand, the loss of function behavior of CK2 $\alpha$-TikREK would suggest that the internal deletion might, by itself, be sufficient to impair activity.

\section{Analysis of dropout clones:}

While the loss of function behavior of TikR and TikREK was unambiguous, this was not the case for Tik and CK2 $\alpha-M K$. In order to eliminate the possibility of these variants are themselves temperature sensitive, dropout experiments were carried out as described above (see Methods). In brief, cells co-expressing variants of Drosophila CK2 in combination with yCKA2 were grown under non-selective conditions to isolate clones that had dropped the (rescuing) yCKA2 plasmid. These strains would thus be 'rescued' by only the dCK $2 \alpha$ variants. Of the various combinations tested, only CK2 $\alpha-\mathrm{MK}$ and CK2 $\alpha$-ED were 


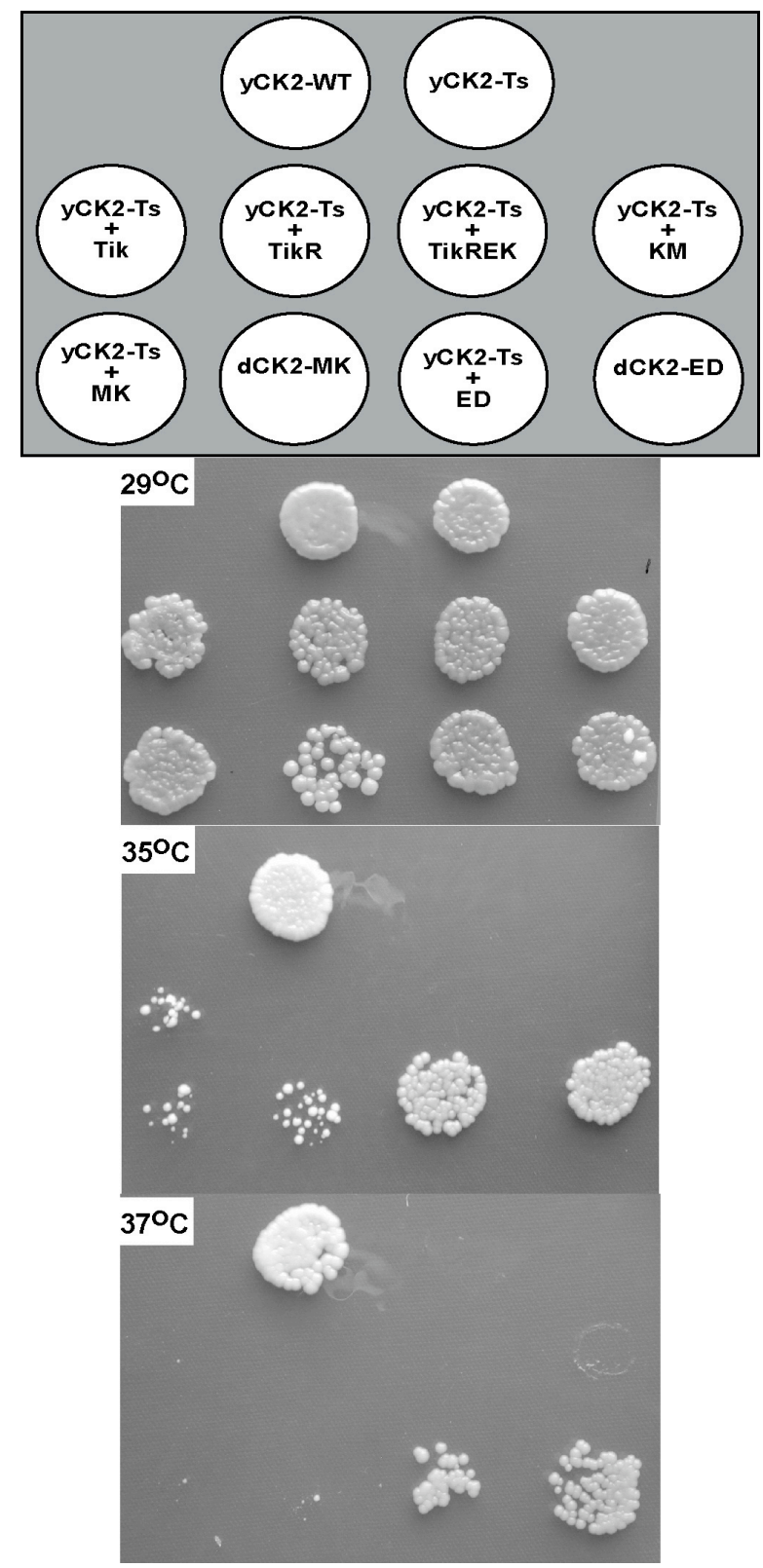

Figure 23: Analysis in yeast rescued by Ts alleles of $\mathrm{dCK} 2 \alpha$ : The ability of CK2 $\alpha-\mathrm{Tik}$, $\mathrm{CK} 2 \alpha-\mathrm{MK}$ and $\mathrm{CK} 2 \alpha-E D$ to rescue the growth arrest of $\mathrm{YDH} 8$ at $35^{\circ} \mathrm{C}$ was tested. As expected, YDH8 strain grew efficiently at the permissive temperature, reflecting full activity of yCK2 $\alpha$-Ts allele. Consistent with the previous analysis, CK2 $\alpha-$ Tik and CK2 $\alpha-M K$ did not fully rescue the growth arrest of YDH8 at $35^{\circ} \mathrm{C}$, indicating that they are non-functional, unlike I CK2 $\alpha-E D$. In addition, CK2 $\alpha-T i k$, CK $2 \alpha-T i k R E K$ and CK2 $\alpha-K M$ also didn't rescue the lethality at $35^{\circ} \mathrm{C}$. Next, dropout clones for CK2 $\alpha$ MK and CK2 $\alpha-E D$ were obtained and tested for growth at $29^{\circ} \mathrm{C}$, as well as at $35^{\circ} \mathrm{C}$ and $37^{\circ} \mathrm{C}$ along with the rest of the constructs and found that CK2 $\alpha-M K$, but not CK2 $\alpha-E D$, is Ts for activity. 
competent to rescue when present in the cells by themselves. At face value, this strongly suggests that Tik does not possess levels of activity that are sufficient for cell growth. This distinct behavior of Tik, does not reflect subtle differences in transcription/translation, because this construct was generated in a manner identical to those that express CK2 $\alpha-\mathrm{MK}$ or CK2 $\alpha-E D$.

However, strains harboring CK2 $\alpha-$ Tik, CK2 $\alpha-$ TikR, CK2 $\alpha-$ TikREK and CK2 $\alpha-K M$ constructs were unable to drop the yCKA2 plasmid, thus indicating that none of these variants possess levels of CK2 activity that are sufficient for cell growth. On the other hand, dropout clones for $\mathrm{CK} 2 \alpha-\mathrm{MK}$ and $\mathrm{CK} 2 \alpha-\mathrm{ED}$ were obtained and tested for growth at $29^{\circ} \mathrm{C}$, as well as at $35^{\circ} \mathrm{C}$ and $37^{\circ} \mathrm{C}$. Both strains grew akin to wild type yeast (RPG41-1a) at $29^{\circ} \mathrm{C}$. Cells rescued by $\mathrm{CK} 2 \alpha-\mathrm{MK}$ grew weakly at $35^{\circ} \mathrm{C}$, whereas they displayed a complete growth arrest at $37^{\circ} \mathrm{C}$. In contrast, cells rescued by CK2 $\alpha-E D$ exhibited equivalent levels of growth at $35^{\circ} \mathrm{C}$ and $37^{\circ} \mathrm{C}$. Thus, it appears that CK2 $\alpha-M K$, but not CK2 $\alpha-E D$, is Ts for activity.

\section{Mean generation time:}

Although previous experiments confirmed that CK2 $\alpha-\mathrm{MK}$, but not CK2 $\alpha-E D$, could not rescue the lethality of YDH8, growth on plates is a semi-quantitative method to measure the complementation efficiency. Thus, the mean generation time (MGT) was assessed to provide for a quantitative analysis (Fig. 24). The MGT is a measure of the average time required for cells to undergo one cell division. It has previously been shown that CK2 is required for cell cycle progression. Consequently, cells in which CK2 is depleted display unique phenotypes that are hallmarks of underlying defects in cell cycle progression. For instance, cka2-null strains display cell cycle arrest at the G1/S and G2/M transitions, which results in elongated pseudomycelial cells, that are referred to as 'shmoos' (based on the Li'l Abner's cartoon character) (MacKay, 1974). In contrast, cka1-null strains display defects 
A

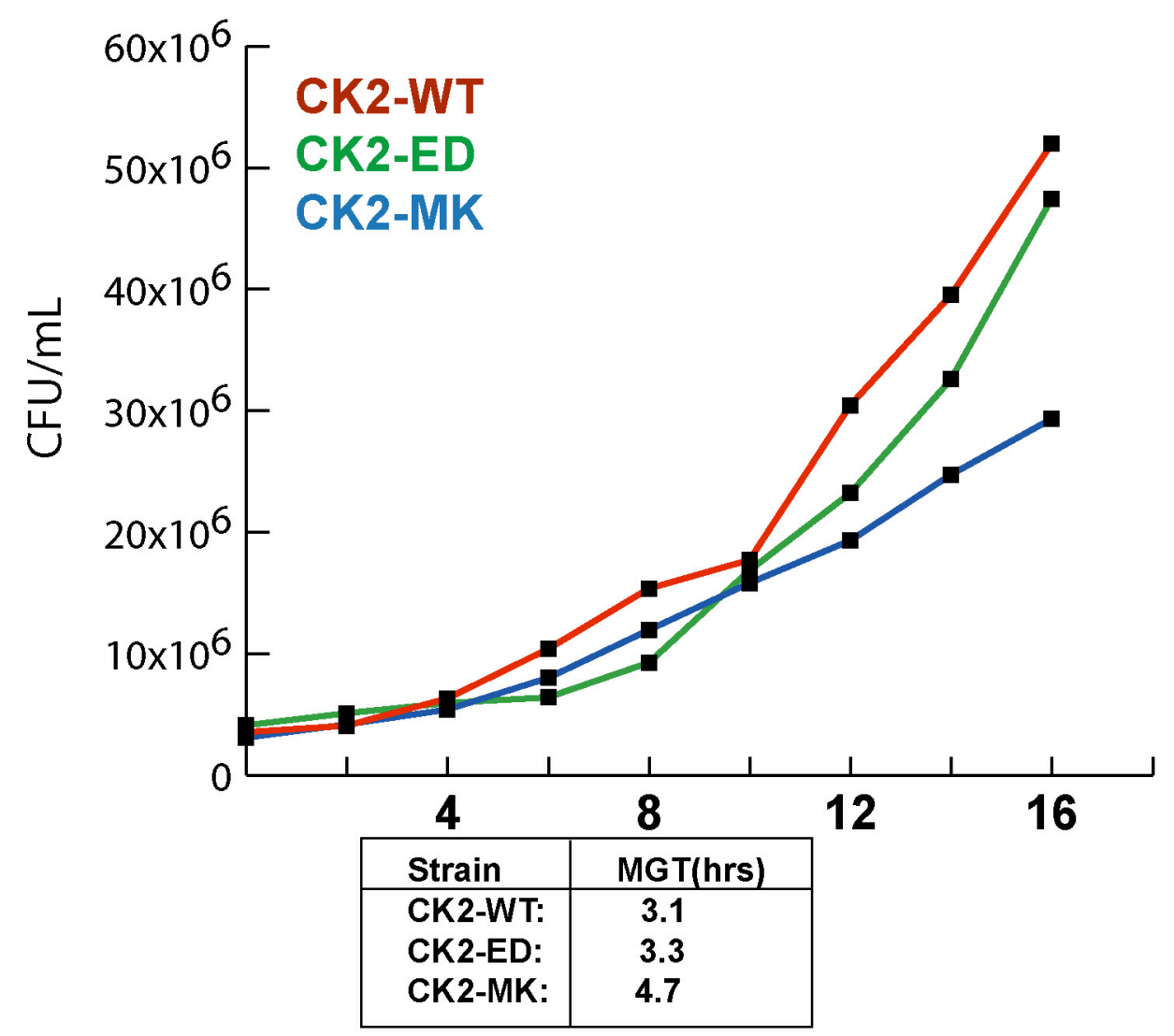

B

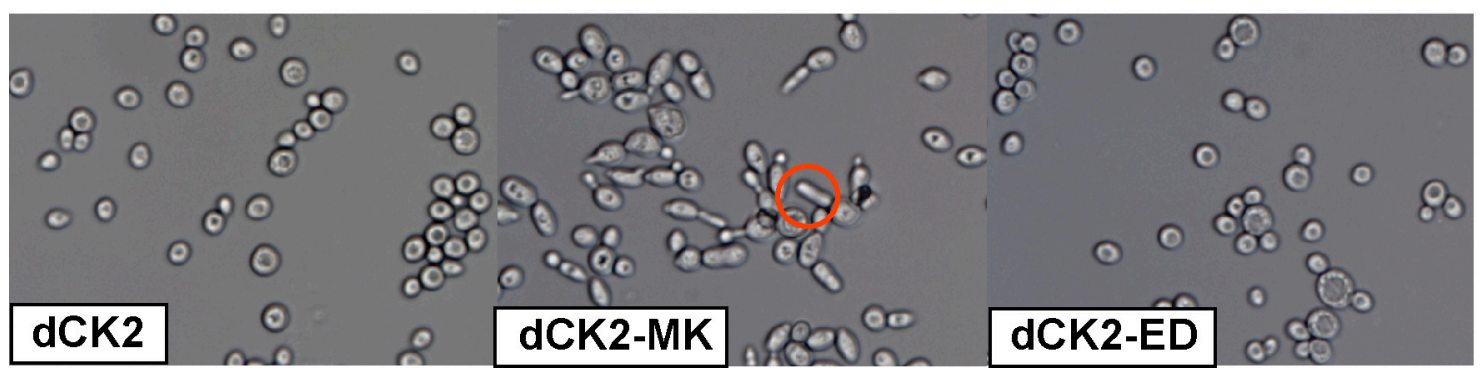

Figure 24: The mean generation time and terminal phenotypes of strains rescued by dCK2 $\alpha-M K$ and dCK2 $\alpha$-ED: A) The mean generation time (MGT) of dCK2 $\alpha-M K$ and dCK2 $\alpha$-ED were measured to assess the efficiency of the complementation as compared to that with wild type dCK2 $\alpha$. The growth rate of CK2 $\alpha$-ED was found to be comparable to that with WT CK2 $\alpha$, whereas CK2 $\alpha-M K$ exhibited attenuated growth-rate. B) Terminal phenotypes of CK2 $\alpha-M K$ and CK2 $\alpha-E D$ were compared to CK2 $\alpha-W T$. It was found that CK2 $2 \alpha-W T$ and CK2 $\alpha-E D$ displayed wild type phenotypes, whereas CK2 $\alpha-M K$ cells displayed the 'shmoo' phenotype, which is an indicative of cell cycle arrest. 
in cell polarity, and result in enlarged, but spherical cells, which resemble the 'Orb' mutants that were originally described in fission yeast (Verde F, 1995). These defects ultimately elicited attenuated growth rates, which altered the MGT.

Thus, the MGT's of dCK2 $\alpha-M K$ and dCK2 $\alpha-E D$ were measured to assess the efficiency of the complementation as compared to that with wild type dCK2 $\alpha$. Cells were grown in YPG at $29^{\circ} \mathrm{C}(\mathrm{PT})$ and processed as described above (see Methods). The growth rate of CK2 $\alpha$-ED was found to be comparable to that with WT CK2 $\alpha$, whereas CK2 $\alpha-\mathrm{MK}$ exhibited attenuated growth-rate. Given the role of CK2 in cell cycle progression, the attenuated growth-rate of CK2 $\alpha-\mathrm{MK}$ confirms that this variant is not catalytically 'inactive', but possesses residual activity, which is sufficient to support yeast viability. Information on the critical amount of CK2 that is not rate limiting for cell division is currently unknown. Therefore it is formally possible that the ability of CK2 $\alpha-M K$ to support viability (in yeast) represents overexpression of a catalytically attenuated CK2 variant. This might not apply to Drosophila.

\section{Terminal phenotype:}

The slow-growth phenotype of CK2 $\alpha-\mathrm{MK}$ suggests that this variant is catalytically compromised. This finding also suggests that CK2 $\alpha-M K$ might display a phenotype as seen in other cell cycle mutants of CK2 (Fig. 24). As described in the previous section, CK2 depletion in yeast leads to two distinct phenotypes, depending on which stage the cell cycle arrest has occurred. Thus, terminal phenotypes of the yeast cells harboring the CK2 $\alpha-\mathrm{MK}$ and CK2 $\alpha$-ED were compared to cells expressing CK2 $\alpha-W T$. For this analysis, cells were temperature shifted to the NPT $\left(35^{\circ} \mathrm{C}\right)$, and cell morphologies were assessed. It was found that CK2 $\alpha-$ WT and CK2 $\alpha$-ED displayed wild type phenotypes, whereas CK2 $\alpha-$ MK cells displayed the 'shmoo' phenotype. It is important to note that not all cells display this 'shmoo' 
phenotype. This reflects the fact that these studies were conducted with asynchronous cells. It has been previously found for CK2 and other cdc mutants, that asynchronous cells that are shifted to the NPT display both 'shmoo' and 'non-shmoo' cells at approximately equal frequencies. However, when cells are synchronized, they coordinate cell cycle progression in a precise manner for at least for 5-7 cell cycles. Under these conditions, all cells display a G1-arrest, which is manifest as 'shmooing' cells. Thus it can be surmised that the low level activity of CK2 $\alpha-\mathrm{MK}$ is likely to be rate limiting for the $\mathrm{G} 1$ phase of the cell cycle.

\section{Expression and stability of CK2 Variants:}

The previous result suggested that cells rescued by CK2 $\alpha-\mathrm{MK}$ alone exhibited an attenuated growth-rate and were likely to be compromised for catalytic activity. However, such effects could also be manifest in the event that this variant was expressed at lower levels or was unstable. Thus steady-state expression levels of dCK2 $\alpha-\mathrm{TiK}, \mathrm{dCK} 2 \alpha-\mathrm{MK}$ and dCK2 $\alpha-\mathrm{ED}$, were assessed by Western Blot analysis, and compared to those of WT dCK2 $\alpha$ (Fig. 25). Because cells rescued by dCK2 $\alpha-$ TiK alone cannot be isolated, all of these studies were conducted in yeast that harbored a Ts-variant of yCK2. Thus these cells express both, yCK2 and dCK2. A distinction between these was facilitated by the observation that the polyclonal antibody raised against Drosophila CK2 does not cross-react with any yeast CK2 subunits (Bidwai et al., 1992). I find that all variants of dCK2 $\alpha$ were expressed at comparable levels (compare Gel and WB in Fig. 25). No immunoreactivity was observed in cells that did not express $\mathrm{dCK} 2 \alpha$, and thus the band observed in lanes 3-6 represent a specific interaction with dCK $2 \alpha$ or its variants. These studies suggest that the attenuated

growth-rate of $\mathrm{CK} 2 \alpha-\mathrm{MK}$ is not a reflection of attenuated expression levels. 


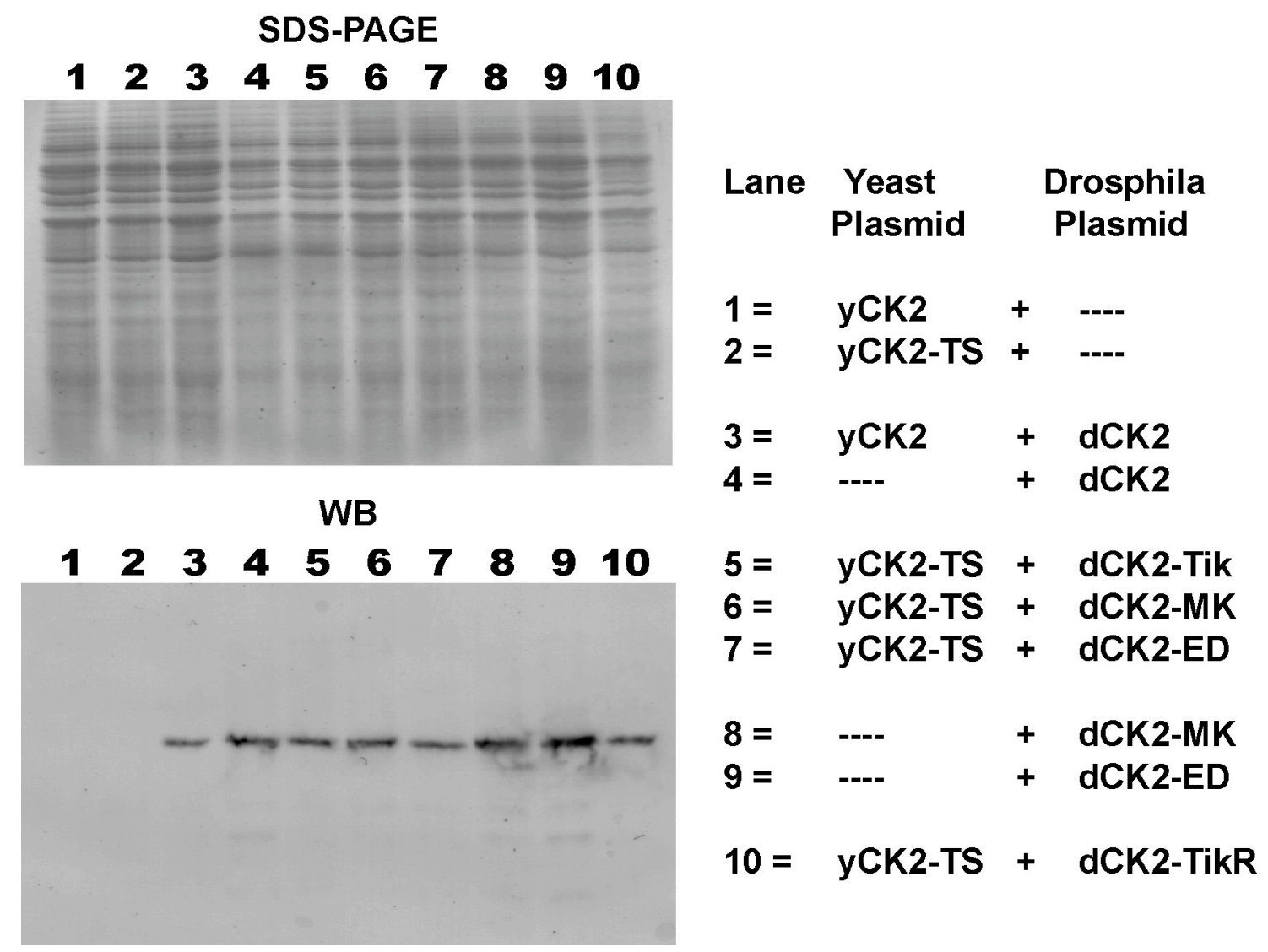

Figure 25: Expression and stability of dCK2 $\alpha$ constructs: Thus steady-state expression levels of dCK $2 \alpha-\mathrm{TiK}, \mathrm{dCK} 2 \alpha-\mathrm{MK}$ and dCK $2 \alpha-\mathrm{ED}$, were assessed by Western Blot analysis, and compared to those of WT dCK2 $\alpha$. I find that all variants of dCK $2 \alpha$ were expressed at comparable levels. 


\section{Protein-protein interactions:}

The interactions between $\mathrm{CK} 2 \alpha$ and $\mathrm{CK} 2 \beta$ are required for in vivo function in Drosophila. Since the eventual goal was to test for the in vivo effects of Tik and its variants in Drosophila, the affinity of interaction between CK2 $\alpha$-variants and CK2 $\beta$ were tested. To evaluate this, fusion proteins were generated between $d C K 2 \alpha$ and its variants with the activation domain (AD) of Gal4 (see Methods), and these were tested for interaction with a fusion between CK2 $\beta$ and the DNA binding (DB) domain of Gal4 (Fig. 27). Mating type twohybrid assays (Brent and Finley, 1994) were conducted, to measure the strength of interaction. The AD-fusions were transformed into the haploid yeast strain pJ69-4 $\alpha$ and the DB-fusion was transformed into strain pJ69-4a. Mating between these two haploid cells generated diploid cells, which express all possible combinations. These diploids were tested for the induction of the two hybrid reporter genes, HIS3, ADE2 and LacZ. Thus productive/stable protein-protein interactions result in growth in media lacking histidine or adenine. All relevant strains were grown, and the same numbers of cells were spotted on selective media.

As expected, haploid cells expressing AD-CK2 $\alpha$ fusions did not grow in $\mathrm{His}^{-}$or $\mathrm{Ade}^{-}$ medium (Fig. 26). In contrast, haploid cells expressing DB-CK2 $\beta$ exhibited weak and sporadic (baseline) growth. This baseline and sporadic growth has been noted previously in our laboratory, and is thought to reflect leaky expression due to the internal acidic region in CK2 $\beta$. In contrast, diploids co-expressing CK2 $\beta$ plus CK2 $\alpha$ displayed robust growth on either selective media. Qualitatively, similar growth rates were observed with cells that expressed CK2 $\beta$ with Tik, CK2 $\alpha-\mathrm{MK}$ or CK2 $\alpha-E D$, suggesting that these three variants might not be perturbed for this interaction. The strong and equivalent interaction between CK2 $\alpha-$ Tik, CK2 $\alpha-\mathrm{MK}$ or CK2 $\alpha-E D$ with CK2 $\beta$ was expected since neither of the substitutions,

$M^{161} K$ and $E^{165} D$, are located near the $\alpha / \beta$ interaction interface. On the other hand, neither 
CK2 $\alpha$-TikR nor CK2 $\alpha$-TikREK interacted with CK2 $\beta$, i.e., these cells lacked the ability to grow on $\mathrm{His}^{-}$or $\mathrm{Ade}^{-}$media. It is important to note the few residual colonies that grew on His plates most probably due to the leaky His expression. As mentioned earlier, His plates contain 3-AT histidine blocker due to this leaky expression. The concentration of 3-AT in these plates was $0.5 \mathrm{mM}$ and it is possible to use higher concentrations to suppress the background growth entirely. The CK2 $\alpha$-TikR and CK2 $\alpha$-TikREK results were expected since neither variant is predicted to bind to CK2 $\beta$ due to the internal deletion. These results confirm that the internal deletion (in TikR) perturbs folding and impairs interaction with CK2 $\beta$. The interactions were also confirmed by assessing activity of the third reporter gene, LacZ. The yeast strain pJ69-4 contains a lacZ gene under control of the Gal7 promoter, which enables quantitative measurements of protein-protein interaction strengths/affinity. Consistent with the (qualitative) results on $\mathrm{His}^{-}$and $\mathrm{Ade}^{-}$media, co-expression of CK2 $\alpha-\mathrm{Tik}_{\text {, }}$ CK2 $\alpha-M K$, and CK2 $\alpha-E D$ with CK2 $\beta$ elicited robust LacZ activity (Fig. 26). Consistent with the molecular model and prediction of the structural perturbations, co-expression of CK2 $\alpha$ TikR or CK2 $\alpha$-TikREK with CK2 $\beta$ did not result in any detectable LacZ activity. Therefore, the deletion in the TikR allele perturbs the structure, which in turn abrogates interaction with CK2 $\beta$. The observations that neither CK2 $\alpha-$ TikR nor CK2 $\alpha$-TikREK rescue yeast or interact with CK2 $\beta$ appear relevant to the in vivo behavior of TikR. For example, TikR/TikR flies are larval1 lethal, whereas TikR/+ flies are viable and display subtle clock defects as compared to Tik/+ flies. The possibility thus arises that the 'revertant' behavior of TikR might reflect its inability to interact with CK2 $\beta$ and poison the holoenzyme. 
A

\section{AD-CK2 $\alpha /$ Variants}

\section{BD-CK2 $\beta$}

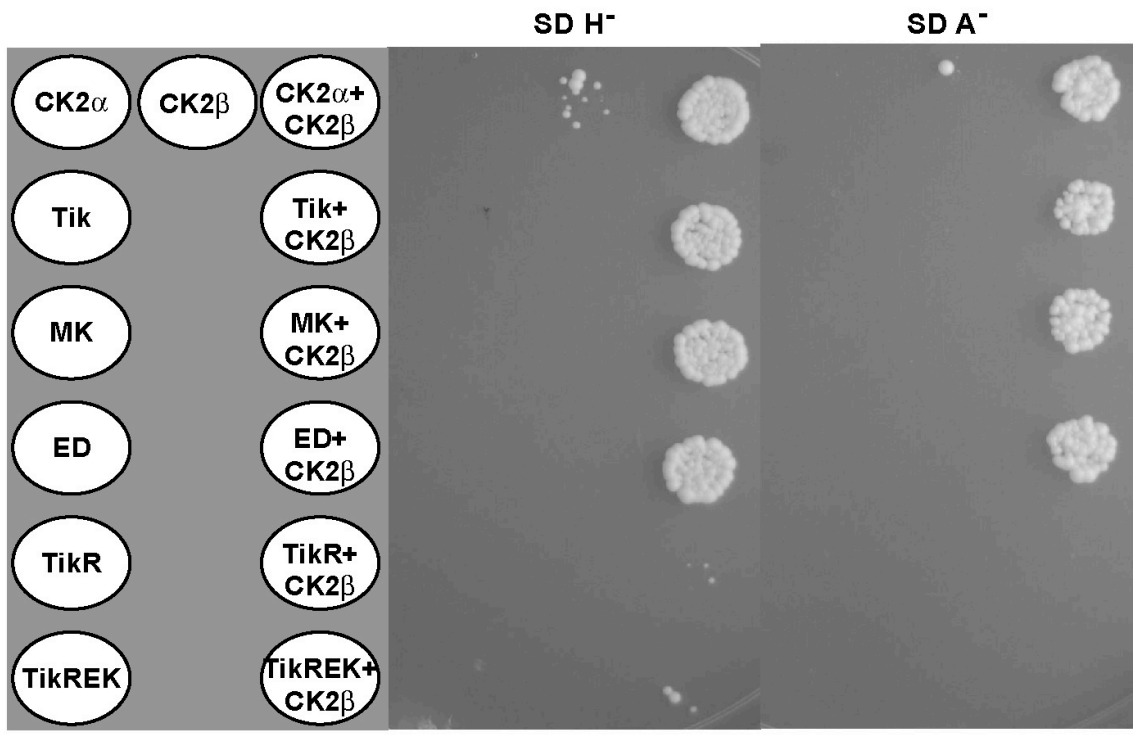

B

\begin{tabular}{|lcc|c|}
\hline \multicolumn{2}{|c|}{ Interaction Tested } & LacZ Activity \\
\hline$\alpha$ & + & $\beta$ & + \\
Tik & + & $\beta$ & + \\
MK & + & $\beta$ & + \\
ED & + & $\beta$ & + \\
TikR & + & + \\
TikREK & + & - \\
\hline
\end{tabular}

Figure 26: Interaction of dCK2 $\alpha$ variants with CK2 $\beta$ : The affinity of interaction between CK2 $\alpha$-variants and CK2 $\beta$ were tested. A) Fusion proteins were generated between $d C K 2 \alpha$ and its variants with the activation domain (AD) of Gal4 and these were tested for interaction with a fusion between CK2 $\beta$ and the DNA binding (DB) domain of Gal4. B) As expected, haploid cells expressing AD-CK $2 \alpha$ fusions did not grow in His ${ }^{-}$or Ade- medium and diploids co-expressing CK2 $\beta$ plus CK2 $\alpha$ displayed robust growth on either selective media. C) Consistent with the (qualitative) results on His and Ade media, co-expression of CK2 $\alpha-$ Tik, CK2 $\alpha-M K$, and CK $2 \alpha-E D$ with CK2 $\beta$ elicited robust LacZ activity 


\section{In vivo analysis in D. melanogaster:}

The yeast studies confirmed that CK2 $\alpha-$ Tik, CK2 $\alpha-\mathrm{MK}$ are compromised for CK2 activity and can not rescue yeast. On the other hand, CK2 $\alpha$-ED behaves more analogous to CK2 $\alpha-$ WT and rescues the yeast strains, which are null for CK2. Furthermore, proteinprotein interactions reveal that all three variants are able to interact with CK2 $\beta$ albeit at different efficiencies. These results suggest that CK2 $\alpha-E D$ is catalytically active and able to interact with CK2 $\beta$. However, the possibility remains that this substitution might perturb the interaction between CK2 and PP2A, an aspect not analyzable in yeast, since $E^{165}$ is not conserved in the $\alpha$ or $\alpha$ ' subunits of yeast CK2. Therefore, it is necessary to conduct studies on these variants in Drosophila to provide a better representation of the impact of these substitutions.

Previous studies from our laboratory have shown that reducing levels/activity of CK2 elicit eye and bristle defects. Specifically, expression of a CK2-RNAi construct, or one encoding for Tik (a catalytically dead CK2 subunit) leads to ectopic bristles and rough eyes, which manifest due to the specification of excess SOPs and R8 cells, respectively. These neural defects are similar to those upon loss of $\mathrm{E}(\mathrm{spl})$ or loss of inhibitory $\mathrm{N}$ signaling. Since CK2 regulates $\mathrm{E}(\mathrm{spl}) \mathrm{M} 8$ via phosphorylation, the possibility arises that the activity of this protein kinase is an essential regulatory step during neurogenesis. These observations thus suggest that bristle and eye development are sensitive to perturbed levels/activity of CK2. In addition, it is likely that phosphorylation of M8 is also regulated via the activities of enzymes that act opposite to CK2. Such an enzyme might be PP2A, a phosphatase that is expressed in the eye and one that has previously been shown to be involved in bristle and eye development. Given that SOP/R8 selection both involve similar components of inhibitory $\mathrm{N}$ signaling, analysis of these CK2 variants during these two developmental programs might uncover a role for PP2A. In the event that these studies suggest a nexus 
between CK2, PP2A and E(spl)M8. It will provide an unprecedented opportunity to begin a systematic analysis of the role of these proteins during neurogenesis.

\section{Generation of transgenic flies:}

The GAL4-UAS expression system was employed to express CK2 $\alpha-M K$ and CK2 $\alpha$-ED in Drosophila. CK2 $\alpha-\mathrm{MK}$ and CK2 $\alpha$-ED cDNA's were subcloned into the Drosophila transformation vector pUAST as described in the methods. pUAST contains the mini-white gene whose germline insertion into the fly genome leads to red eye color (henceforth referred to as $\left.w^{+}\right)$. $w^{1118}$ embryos were injected with the constructs by Best Gene Inc. Multiple transgenic lines were generated for each variant. Specifically, 9 independent lines for CK2 $\alpha-M K$ and 17 independent lines for CK2 $\alpha-E D$ were generated.

\section{Mapping of transgenic flies:}

Since the transgene insertions are random, it is necessary to first identify which chromosome the insertion has occurred in. In order to map the location of the insertions, standard protocols were followed (Fig. 27). Specifically, first $w^{+}$flies for each line (male or virgin female) were crossed with flies of the genotype $\mathrm{CyO} /+$. Then, progeny of this cross that are $w^{+}$and Cy $0+$ were then crossed to $w^{1118}$ the $2^{\text {nd }}$ chromosome. However, if the segregation of $\mathrm{CyO}$ from $w^{+}$is not $100 \%$, this indicates that the transgene is most probably on the $3^{\text {rd }}$ chromosome. Lastly, if the progeny contains only red-eyed females, then the transgene is likely to be located on chromosome X. Subsequently; balanced stocks of each transgenic line were established using appropriate balancer chromosomes for X, II, III.

First, the ability of these constructs to elicit neural defects in the eye and bristle was tested. Subsequently; the ability of these constructs to rescue the rough and reduced eye phenotype of a hypersensitive allele of $N\left(N^{s p l}\right)$ was tested. For both of these studies, UAS$C K 2 \alpha-M K$ and UAS-CK $2 \alpha-E D$ were compared to UAS-CK $2 \alpha-T i k$ and UAS-CK $2 \alpha-W T$. 


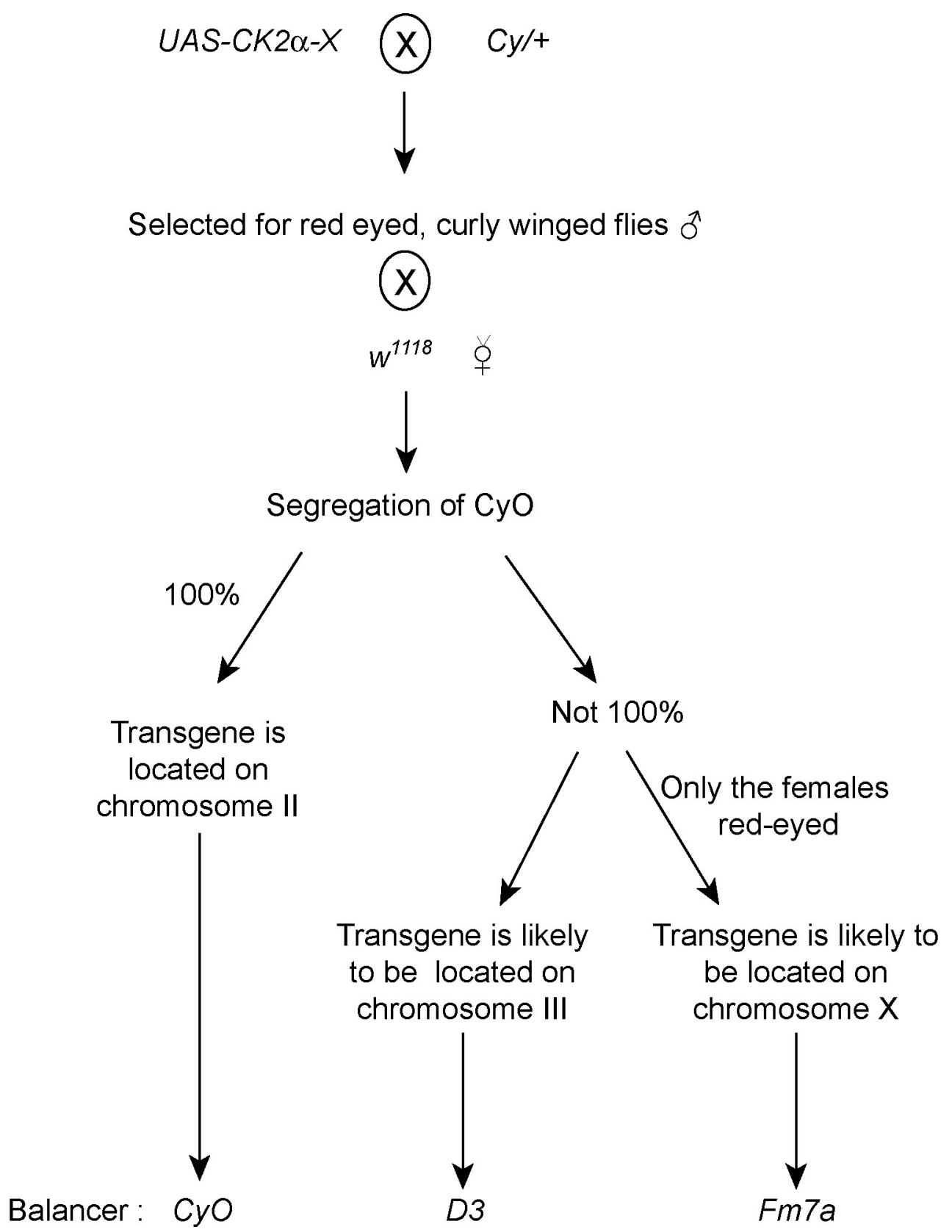

Figure 27: Mapping and balancing of the transgenic lines: Standard mating protocol was employed to map the location of the transgenic insertions. 


\section{Ability to elicit bristle defects in wild type flies:}

Bristles are mechanosensory organs that can be found as macrochaetaes (the large bristles) or microchaetaes (the small bristles), whose position and numbers are precisely regulated by various signaling pathways including $\mathrm{N}$ signaling. In brief, a single cell, the SOP, is selected from a group of equipotent cells in the PNC and undergoes multiple asymmetric cell divisions to give rise to four sister cell fates, neuron, sheat, shaft and socket (Fig. 16). All of these asymmetric cell divisions are under the control of $\mathrm{N}$ signaling. Therefore, compromising $\mathrm{N}$ activity elicits ectopic and duplicated bristles. In contrast, hyperactivity of $\mathrm{N}$ elicits missing/split bristles and ectopic socket cell formation. Previous studies from our laboratory suggest that reducing the levels/activity of CK2 lead to ectopic bristles, which indicate compromised N signaling (Bose et al., 2006; Karandikar et al., 2004). Based on the yeast studies, CK2 $\alpha-\mathrm{MK}$ was found to be catalytically compromised and it is likely that this variant will behave in an analogous manner in flies. Therefore, it is expected that overexpression of CK2 $\alpha-M K$ during SOP selection will compromise $\mathrm{N}$ signaling. If this is the case, excess SOP's are expected to arise from each PNC. These SOP's will then go through differentiation independent of each other and manifest as ectopic and closely positioned bristles. In contrast to CK2 $\alpha-\mathrm{MK}, \mathrm{CK} 2 \alpha-\mathrm{ED}$ displayed catalytic activity that is analogous to wild type. However, as stated above, the $\mathrm{E}^{165}$ residue is not conserved in yeast, unlike Drosophila. Therefore, it is necessary to test UAS-CK2 $\alpha-E D$ variant in Drosophila, which might provide a better model system for CK2 $\alpha-E D$.

\section{UAS-constructs encoding CK2 $\alpha-\mathrm{WT}$, CK2 $\alpha-$ Tik, CK2 $\alpha-\mathrm{MK}$ and CK2 $\alpha$-ED were} tested for their ability to elicit bristle defects in otherwise wild type flies. Expression of the transgenes was achieved with the Gal4 drivers, scaGal4 or G455.2. The former driver is active in all PNC's that give rise to the notal macrochaetes, whereas the latter is restricted to the PNC's that give rise to the four scutellar macrochaetes. It has previously been found 
that expression of $\mathrm{E}(\mathrm{spl})$ variants with either driver elicits bristle loss, whereas expression of CK2-RNAi/DN elicits ectopic bristles.

Crosses were established at $24^{\circ} \mathrm{C}$ and the notal macrocheate counts were determined. It should be noted that scaGal4 and G455.2 display ectopic bristles on their own. Therefore the baseline defects of these two drivers was first determined and used for comparison to the bristle defects associated with the overexpression of CK2 $\alpha$-variants. In the case of G455.2/+ flies the baseline bristle defects are seen in $\sim 15 \%$, this number is $\sim 35 \%$ for scaGal4/+ flies. As previously described, the expression of UAS-Tik led to ectopic macrochaetes whose penetrance was higher than the baseline (Fig. 28). A similar phenotype was also associated with the expression of either UAS-CK2 $\alpha-M K$ or UAS-CK $2 \alpha-$ $E D$ (Fig. 28). Similar phenotypes are associated with multiple insertions of the UAS-CK2 $\alpha$ MK or UAS-CK $2 \alpha-E D$ constructs (data not shown). The ectopic bristles with CK2 $\alpha-\mathrm{MK}$ were expected given that this variant is also catalytically inactive and is therefore likely to act in a dominant negative manner analogous to Tik. Expression of wild type CK2 $\alpha$ did not elicit ectopic bristles with a penetrance that was different than that of $G 4455 /+$ flies suggesting that increased dosage of CK2 does not perturb $\mathrm{N}$ signaling (Fig. 28). The reason underlying the slight attenuation in the baseline bristle defects of scaGal4/+; UAS-CK2 $\alpha /+$ flies is currently unknown. The elicitation of ectopic bristles with UAS-CK2 $\alpha-E D$ was unexpected since this variant appears to be catalytically active. This result suggests that the ED substitution might also contribute to the dominant negative effects of Tik. The possibility thus arises that aberrant recruitment of PP2A might underlie the effects of CK2 $\alpha$-ED. Increased dosage of PP2A has previously been shown to elicit similar (ectopic) bristle defects. None of the UAS insertions, by themselves, elicited ectopic bristles to an appreciable extent suggesting that transgene expression was required for the observed phenotypes (Fig. 28). Thus analysis with either scaGal4 or G455.2 gave similar results 
A

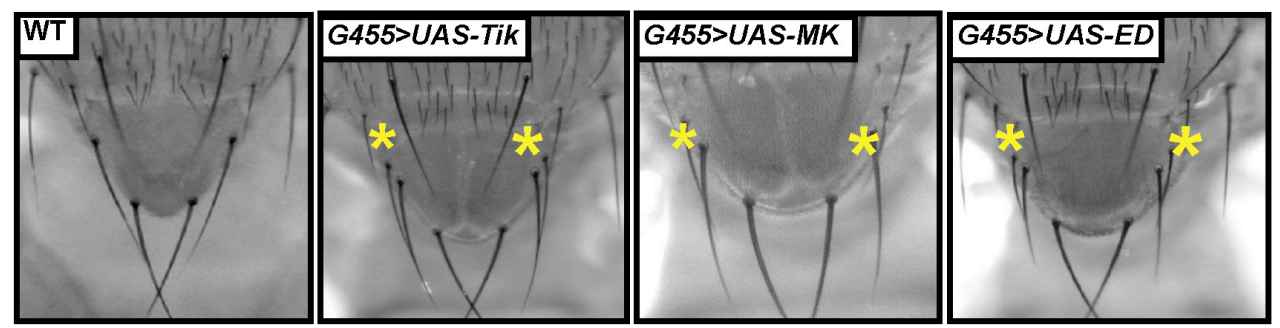

B
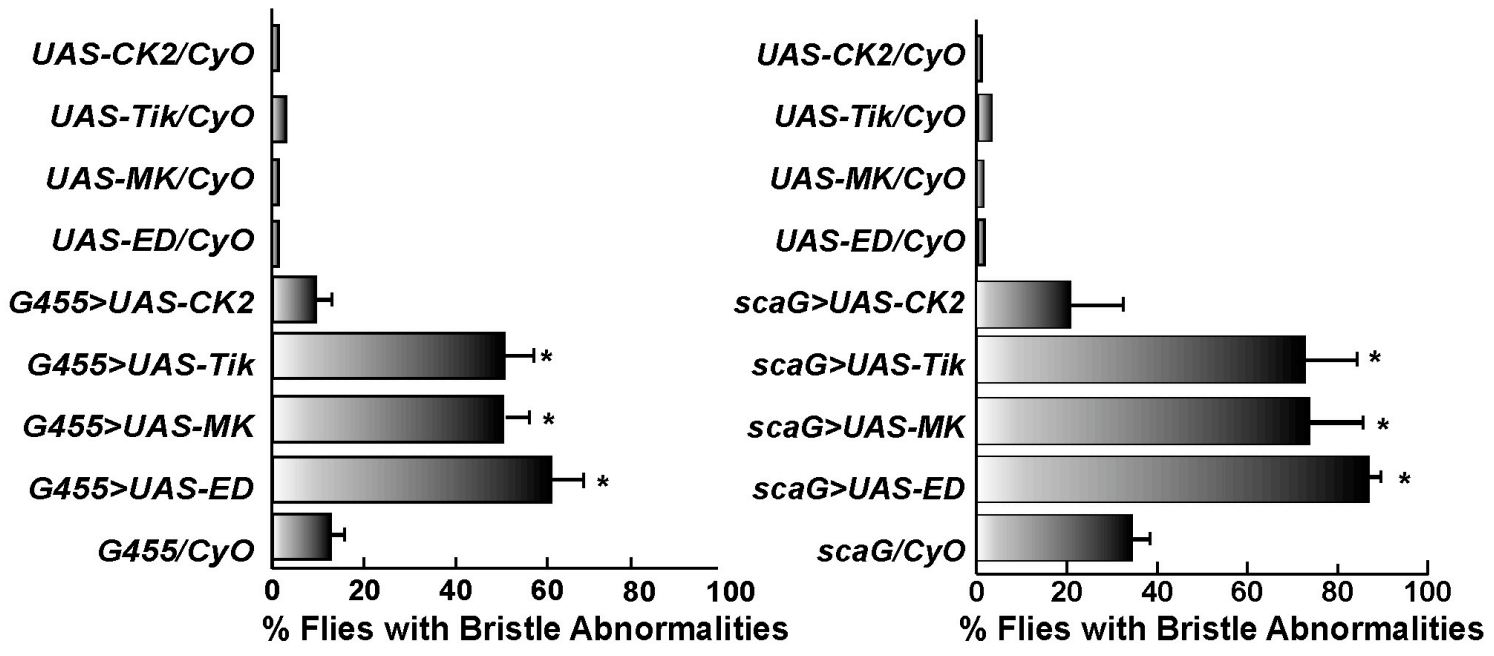

C

\begin{tabular}{lcc}
\multicolumn{3}{c}{ Average Scutellar Bristle counts } \\
& G455.2 & scaGal4 \\
Control & $4.1 \pm 0.07$ & $4.5 \pm 0.1$ \\
UAS-CK2 & $4.1 \pm 0.09$ & $4.4 \pm 0.07$ \\
UAS-Tik & $4.6 \pm 0.03$ & $4.9 \pm 0.3$ \\
UAS-MK & $5.0 \pm 0.1$ & $5.1 \pm 0.2$ \\
UAS-ED & $5.4 \pm 0.07$ & $5.2 \pm 0.2$
\end{tabular}

Figure 28: Ability of UAS-CK2 $\alpha-M K$ and UAS-CK2 $\alpha-E D$ constructs to elicit bristle abnormalities. A) UAS-constructs encoding CK2 $\alpha-W T$, CK2 $\alpha-T i k, C K 2 \alpha-M K$ and CK2 $\alpha$-ED were tested for their ability to elicit bristle defects in otherwise wild type flies. Expression of the transgenes was achieved with the scaGal4 or G455.2 drivers. The CK2 $\alpha$-Tik and CK2 $\alpha-M K$ elicited ectopic bristles (yellow stars). Expression of wild type CK2 $\alpha$ did not elicit ectopic bristles different than that of G4455/+ flies. Surprisingly, expression of CK2 $\alpha$-ED elicited ectopic bristles as well. Student $t$ test was conducted for the statistical analysis of the data ( $p$-value is indicated). 
strengthening the possibility that $U A S-C K 2 \alpha-E D$ does not behave analogous to wild type CK2.

\section{Ability to elicit neural defects in the eye:}

Based on the studies described above, the effects of misexpression of these constructs were assessed in the context on the eye. These studies are based on a body of evidence that inhibitory $\mathrm{N}$ signaling in the bristle and the eye is virtually identical. UASconstructs encoding CK2 $\alpha-\mathrm{WT}, \mathrm{CK} 2 \alpha-\mathrm{Tik}, \mathrm{CK} 2 \alpha-\mathrm{MK}$ and CK2 $\alpha-\mathrm{ED}$ were tested for their ability to elicit dominant eye defects in otherwise wild type flies. Expression of the transgenes was achieved with the Gal4 driver, eyGal4. It has previously been shown that the expression of CK2-RNAi/DN constructs with this driver elicits eye defects such as a rough eye at $29^{\circ} \mathrm{C}$ but not at $24^{\circ} \mathrm{C}$ reflecting the greater expressivity of this driver at the higher temperature (Bose et al., 2006). It was found that at $29^{\circ} \mathrm{C}, U A S-C K 2 \alpha-M K$ elicited eye defects characterized by reduced and rough eyes and occasional ommatidial fusions (Fig. 29). It is important to note that UAS-CK2 $\alpha-M K /+$ flies do not exhibit any such abnormalities (data not shown). On the other hand, UAS-CK $2 \alpha-T i k, U A S-C K 2 \alpha-E D$ and UAS-CK $2 \alpha-W T$ did not lead to detectable eye defects.

\section{Ability to suppress eye phenotypes of $\boldsymbol{N}^{s p l}$ :}

Previous studies suggested that UAS-CK2 $\alpha-M K$ and UAS-CK $2 \alpha-E D$ elicit similar bristle defects. However, the latter variant did not elicit any overt eye defects. Therefore, a sensitized $\mathrm{N}$ background was utilized, with the expectation that under these conditions subtle differences between the CK2 variants might be dissectible. The split allele of Notch $\left(N^{s p l}\right)$ was used for these studies. $N^{s p l}$ harbors a single amino acid substitution $l l{ }^{578}$ to Thr in the extracellular domain, which renders $\mathrm{R} 8$ cells sensitive to the inhibitory $\mathrm{N}$ signaling. This accentuated $\mathrm{N}$ activity impairs specification as well as differentiation of $\mathrm{R} 8$ cells. Because 

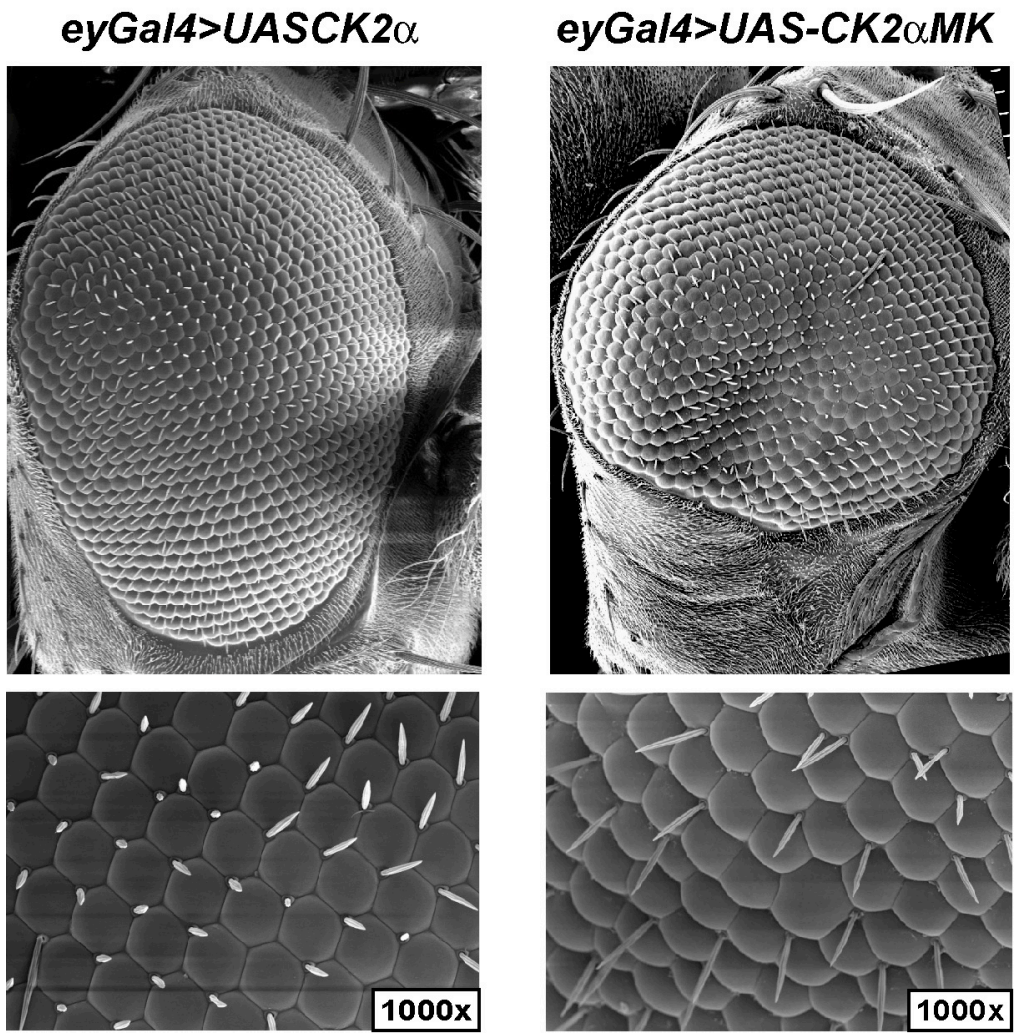

Figure 29: Ability of UAS-CK2 $\alpha-M K$ and UAS-CK2 $\alpha-E D$ constructs to elicit bristle abnormalities: UAS-constructs encoding CK2 $\alpha-W T$, CK2 $\alpha-T i k, C K 2 \alpha-M K$ and CK2 $\alpha$-ED were tested for their ability to elicit dominant eye defects in otherwise wild type flies. Expression of the transgenes was achieved with the Gal4 driver, eyGal 4 at $29^{\circ} \mathrm{C}$. It is found that UAS-CK $2 \alpha-M K$ elicited eye defects. 
the R8 cell is the founding photoreceptor, its aberrant patterning in $N^{s p l}$ leads to a reduced and rough eye. It was previously shown in our laboratory that reducing levels and/or activity of CK2 $\alpha$ suppresses the eye defects of $N^{s p l}$. Given the role of CK2 during $N$, it is conceivable that reducing CK2 levels/activity leads to hypo-phosphorylation of E(spl)M8. This hypo-phosphorylation would attenuate repression by $\mathrm{E}(\mathrm{spl}) \mathrm{M} 8$, which is thought to be the predominant $\mathrm{E}(\mathrm{spl})$ member required for R8 specification. Thus, lowered CK2 activity might antagonize the retinal defects of $N^{s p l}$ by attenuating inhibitory $\mathrm{N}$ activity.

\section{UAS-constructs encoding CK2 $\alpha-\mathrm{WT}, \mathrm{CK} 2 \alpha-\mathrm{Tik}, \mathrm{CK} 2 \alpha-\mathrm{MK}$ and CK2 $\alpha$-ED were} tested for their ability to suppress the eye phenotype of $N^{s p l}$. Since $N$ is X-linked, this suppression is expected to only manifest in male progeny. For these studies, the $N^{\text {spl }}$ allele was combined with $109-68 \mathrm{Ga} / 4$, a driver that is expressed in the MF of the eye disc. In this analysis, virgin females of the genotype $N^{s p l} / N^{s p l} ; 109-68 \mathrm{Gal} / \mathrm{Cyo} ;+/+$ were crossed to males that were heterozygous for an insertion of UAS-CK2 $\alpha$-constructs. Male progeny of this cross were analyzed for eye phenotypes. Males of the genotype $N^{s p l} / Y ; 109$ 68Gal4/CyO and $N^{s p l} / Y ; 109-68 G a l 4 / U A S-C K 2 \alpha$-constructs were compared. The former represent the control, whereas the latter represent the test.

As expected, the control progeny did not show any suppression of the rough and reduced eye of $N^{s p l}$. In contrast, the eye defects of $N^{\text {spl }}$ were suppressed by UAS-Tik, UASCK2 $\alpha-M K$ and UAS-CK2 $\alpha-E D$ (Fig. 30). Most notably, misexpression of UAS-CK2 $\alpha-M K$ led to a strong (but not complete) restoration of the ommatidial phasing/spacing defects that are uniform across the eye field of $N^{s p l} / Y$ flies. Misexpression of UAS-Tik or UAS-CK2 $\alpha-E D$, also suppressed the rough and reduced eye, although to an extent that was lower than that seen with UAS-CK2 $\alpha-M K$. In a similar experimental setup, expression of UAS-CK2-WT was found not elicit suppression of $N^{\text {spl }}$ with any appreciable degree and was thus analogous to 


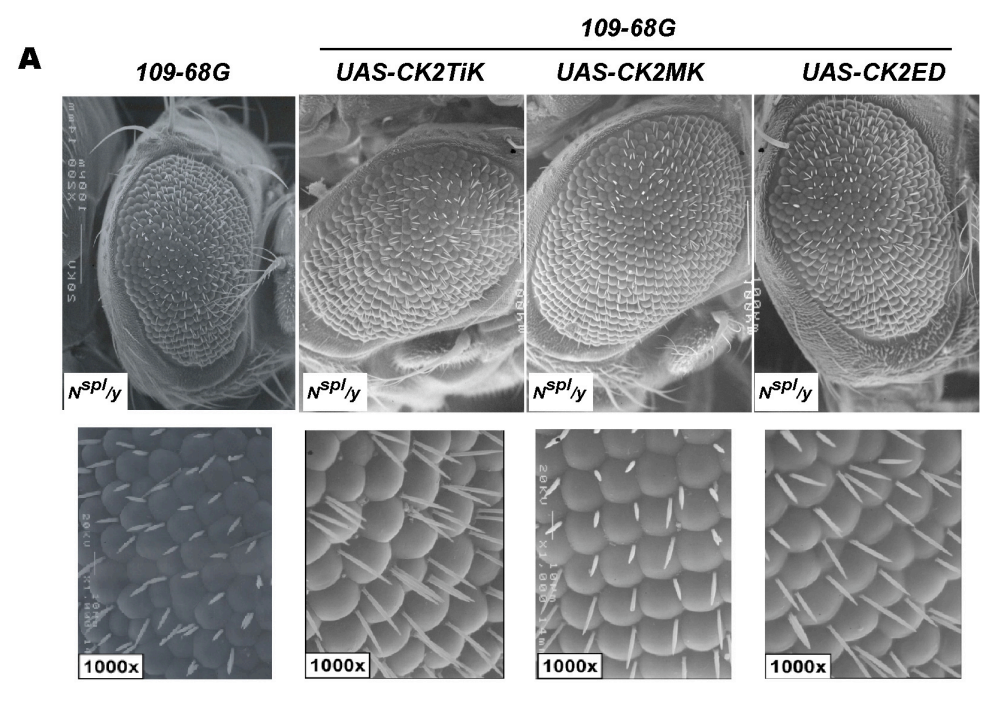

B

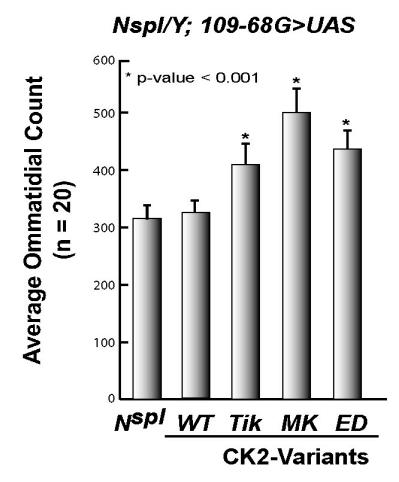

C

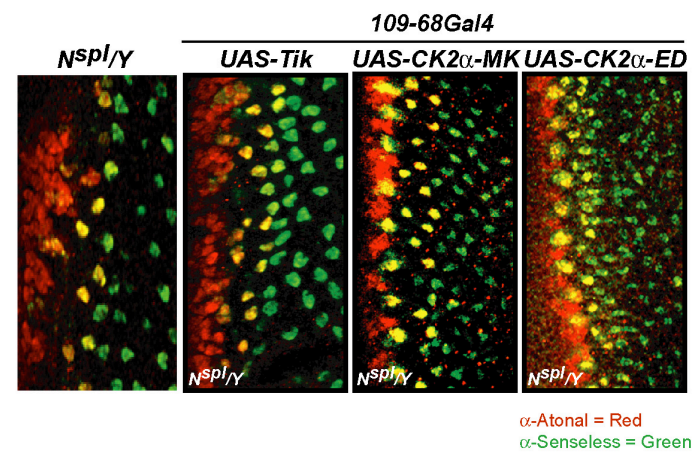

Figure 30: Ability of UAS-CK2 $\alpha-M K$ and UAS-CK2 $\alpha-E D$ constructs to suppress the eye phenotype of $\boldsymbol{N}^{\text {spl }}$. UAS-constructs encoding CK $2 \alpha-W T, C K 2 \alpha-T i k, C K 2 \alpha-M K$ and CK $2 \alpha-E D$ were tested for their ability to suppress the eye phenotype of $N^{\text {spl }}$. A) The eye defects of $N^{\text {spl }}$ were suppressed by UAS-Tik, UAS-CK $2 \alpha-M K$ and UAS-CK $2 \alpha-E D$. B) Statistical analysis suggests that the ommatidial numbers upon expression of CK2 $\alpha-M K, C K 2 \alpha-E D$ and $C K 2 \alpha-T i k$ are significantly higher than those in $N^{s p l} / Y$ males. C) Immunostaining of eye imaginal discs for Atonal (Ato) and Senseless (Sens), revealed that UAS-Tik, UAS-CK2 $\alpha-M K$ and UAS-CK2 $\alpha-E D$ constructs led to phasic restoration of the positions of these cells in the eye disc. 
$N^{s p l}$. It is important to note that female progeny from these crosses did not elicit eye defects, an outcome that was expected since $N^{s p l}$ is recessive (data not shown). To provide a quantitative comparison, the ommatidial counts of $N^{\text {spl }} / Y$ males were compared to those upon expression of the various CK2 variants. $N^{s p l} / Y$ males exhibit an average ommatidial count of 315 . This number represented the baseline against which the comparisons were made. It was found that expression of WT-CK2 325. In contrast, UAS-CK2 $\alpha-M K$ led to 500 UAS-Tik led to 430 and UAS-CK2 $\alpha$-ED led to 410 (Fig. 30). Statistical analysis suggests that the ommatidial numbers upon expression of CK2 $\alpha-\mathrm{MK}, \mathrm{CK} 2 \alpha-\mathrm{ED}$ and CK2 $\alpha$-Tik are significantly higher than those in $N^{s p l} / Y$ males (for $p$ values see Fig. 30 ). In this analysis, the rank order of the three variants was MK>Tik=ED. At face value, these studies suggest that CK2 $\alpha-E D$ does not behave in a manner that is comparable to that of CK2 $\alpha-W T$. One possible reason for the lack of an effect of CK2 $2 \alpha-W T$ could be lack of expressivity. We do not think this is the case because it has previously been shown that the severe rough and reduced eye upon expression of a CK2 $\alpha-R N A i$ construct is neutralized upon coexpression of this CK2 $\alpha$-WT construct (Bose et al., 2006).

The adult eye phenotypes were further assessed by immunostaining of eye imaginal discs for Atonal (Ato) and Senseless (Sens), two proteins that are markers for R8 specification and differentiation, respectively (Fig. 30). In the eye imaginal disc, Ato expression is first detected in all cells anterior to the MF. As the MF progresses, Ato expression becomes restricted to phase shifted cells, the R8 progenitors. Posterior to the MF, these progenitors begin to express the $\mathrm{Zn}$ finger transcriptional factor Sens, whose expression drives differentiation of the R8 photoreceptors. It was therefore expected that the suppression by the CK2 variants should reflect in restored Ato and Sens expression in $N^{s p l} / Y$ males. 
$N^{s p l} / Y$ males display sporadic and inconsistent levels of Ato expression. As a result, the Ato and Sens positive cells posterior to the furrow are mis-patterned. It was found that the expression of UAS-Tik, UAS-CK2 $\alpha-M K$ and UAS-CK $2 \alpha-E D$ constructs restored both R8 specification and differentiation and led to phasic restoration of the positions of these cells in the eye disc.

Ubiquitous expression of CK2 $\alpha-\mathrm{MK}$, but not CK2 $\alpha-E D$, elicits lethality:

The ectopic effects of CK2 $\alpha$-ED were assessed following ubiquitous expression with act5CGal4. As controls CK2 $\alpha-\mathrm{WT}$ and CK2 $\alpha-\mathrm{MK}$ were also tested. As expected, CK2 $\alpha-\mathrm{MK}$ elicited $45 \%$ lethality, whereas CK2 $\alpha$-WT did not. Expression of CK2 $\alpha$-ED with act5CGal4 did not elicit any lethality, suggesting that the ED substitution is not toxic (data not shown). 


\section{CHAPTER-3}

Potential antagonistic roles of CK2 and PP2A in Notch Signaling in Drosophila 


\title{
Potential antagonistic roles of CK2 and PP2A in Notch Signaling in Drosophila
}

\author{
Ezgi Kunttas
}

\begin{abstract}
It is increasingly becoming apparent that dynamic control of phosphorylation and dephosphorylation underlies the proper spatial and temporal activities of many signaling circuits including Notch $(\mathrm{N})$. Emerging evidence suggests that protein kinase CK2 mediates the effects of $\mathrm{N}$ during Drosophila eye and bristle development via phosphorylation of $\mathrm{E}(\mathrm{spl})$ repressors and it has been previously shown that impaired CK2 function compromises lateral inhibition, presumably due to hypo-phosphorylation of $\mathrm{E}(\mathrm{spl}) \mathrm{M} 8$. While these studies implicate a role for $\mathrm{CK} 2$ in the regulation of $\mathrm{E}(\mathrm{spl})$, the identity and/or involvement of a cognate phosphatase remain unknown. Protein phosphatase 2A (PP2A) is one of the candidates thought to be involved in N signaling. We have employed the Gal4-UAS system to assess the role of PP2A during eye and bristle development in wild type and/or $N^{\text {spl }}$ flies. We find that UAS-mts elicits ectopic and missing bristles in $N^{+}$flies, both indicative of a loss of $\mathrm{N}$ signaling. Furthermore, UAS-mts elicits a rough eye phenotype characterized by aberrant ommatidial phasing and/or fusion and loss of the interommatidial bristles. In addition, we find that increased dosage of PP2AC (UAS-mts) suppresses the rough and reduced eye phenotypes of $N^{s p l}$ flies, a result that is also associated with decreased CK2 levels/activity. Together, these results suggest that PP2A activity might attenuate, whereas CK2 augments, inhibitory N signaling. Given the previously described genetic and direct biochemical interactions between CK2 and E(spl)M8/M5/M7, PP2A might serve in an antagonistic manner to CK2.
\end{abstract}




\section{METHODS}

\section{Molecular modeling:}

Swiss-pdb viewer is used to generate the molecular models used in this study. Molecular models for PP2A and its variants were created via 2iae and $1 \mathrm{~b} 3 \mathrm{u}$ pdb files (www.rcsb.org).

\section{Misexpression of transgenic flies:}

The GAL4-UAS system was employed for in vivo analysis with the PP2A constructs for the catalytic subunit, mts. UAS-mts and UAS-dnmts lines were gift from Amita Sehgal, Pennsylvania University, and UAS-mtsRNAi line was purchased from Vienna Drosophila Stock Center (VDRC). Flies were raised at $24^{\circ} \mathrm{C}$ or $29^{\circ} \mathrm{C}$ on a yeast extract and glucose containing media. The Gal4 lines used in these studies were obtained from the Bloomington Stock Center at Indiana University. The Gal4 drivers used in these studies are, G455.2 and scaGal4 (Giebel and Campos-ortega, 1997), eyGal4, act5Gal4. The expression domains of the drivers are shown in Figure 16. Light microscopy was employed for the imaging of the phenotypes. Scanning electron microscopy (SEM) analysis was used for the detailed eye images. For SEM analysis, flies were passed thorough series of dilutions of alcohol series (25\%-50\%-75\%-absolute), and finally passed through Hexamethyldisalizane. The samples were sputter coated, and examined with a JEOL-6400 or Hitachi S-4700 scanning electron microscope. All images were processed by Adobe Photoshop and Adobe Illustrator.

\section{Rescue of $\boldsymbol{N}^{s p l}$ phenotype:}

Gal4-drivers were combined with the $N^{\text {spl }}$ allele as stated previously. Only the male progeny were scored for the analysis, since $N^{\text {spl }}$ is X-linked. Light microscopy and SEM analysis were employed as described above. 


\section{RESULTS AND DISCUSSION}

Previous studies from our laboratory suggested a role for protein kinase CK2 in Notch $(\mathrm{N})$ signaling pathway during eye and bristle development via phosphorylation of $\mathrm{E}(\mathrm{spl})$ repressors. It was shown that misexpression of UAS constructs encoding a dead catalytic subunit of CK2 (a CK2-DN) elicits neural defects, similar to $E(s p l)$ loss of function mutants. Specifically, these constructs elicited ectopic bristles and rough eyes, which are indicative of loss of $\mathrm{N}$ function and compromised lateral inhibition. Therefore, phosphorylation appears to play an important role in the $\mathrm{N}$ pathway. The role for a kinase raises the possibility of involvement of a phosphatase. Protein phosphatase 2A (PP2A) is one of the candidates, which is thought to be involved during $\mathrm{N}$ signaling.

The studies described in this thesis (see Chapter-2.) have confirmed that CK2 $\alpha$-Tik, CK2 $\alpha-M K$, and CK2 $\alpha-E D$ all elicit ectopic bristles and suppress the retinal defects of $N^{s p l}$. None of these effects were observed with the wild type counterpart. Based on these findings, follow-up studies were conducted with PP2A in wild type and $N^{\text {spl }}$ flies.

The studies described in this section are focused on one of the subunits of PP2A, the catalytic subunit encoded by the $m t s$ gene. These studies have involved both gain and loss of function analysis of $\mathrm{mts}$.

\section{In vivo Analysis in D. melanogaster:}

\section{Ability to elicit neural defects in the eye and bristle:}

As stated previously (see Introduction), bristles are precisely positioned, and this patterning involves proper activity of various signaling pathways including $\mathrm{N}$. In addition, the studies described in Chapter-2 suggest that CK2 $\alpha$-ED elicits ectopic bristles that are also 
A

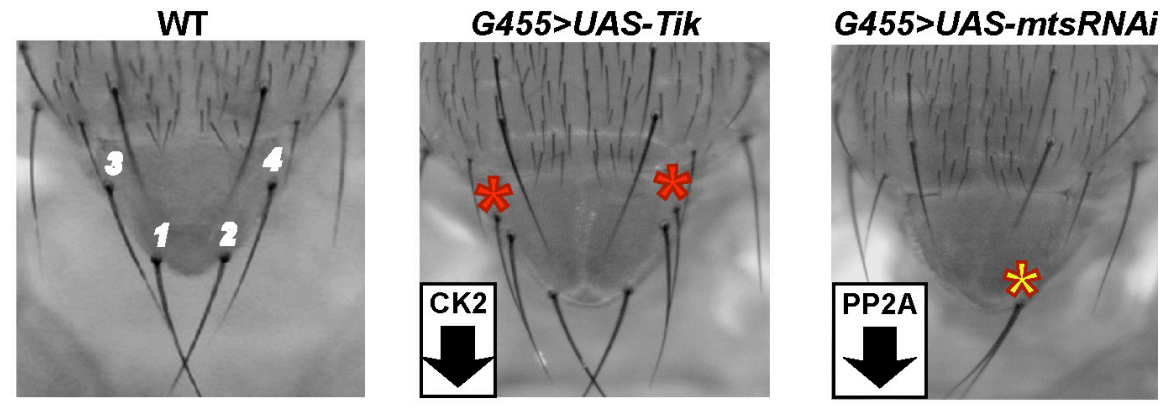

B

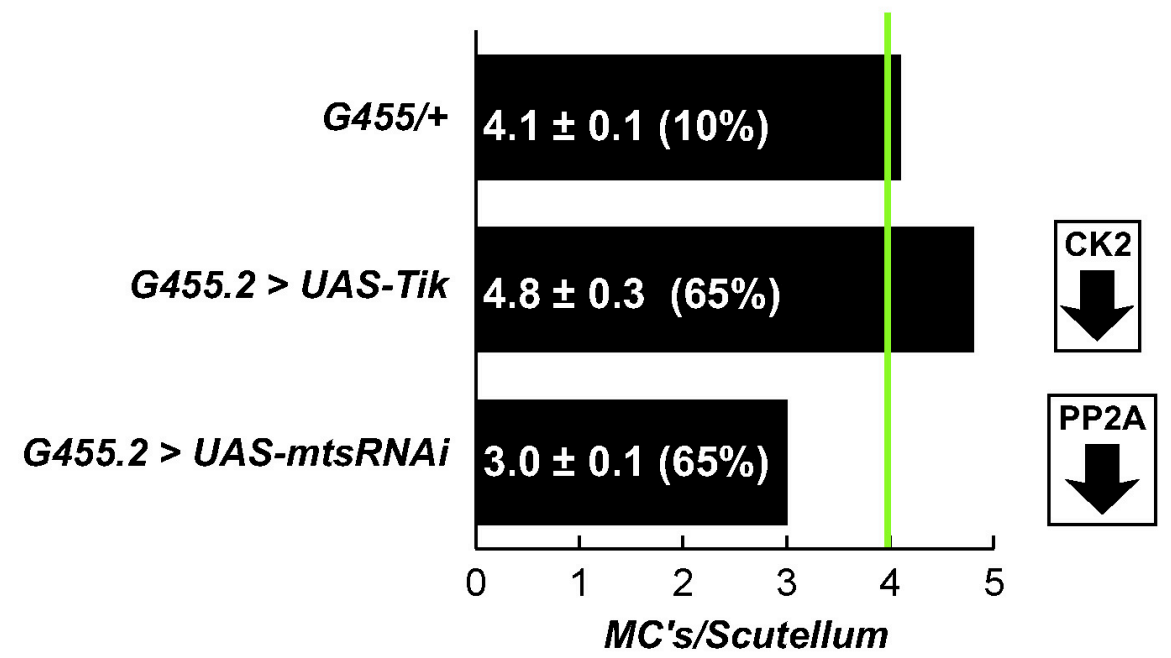

C

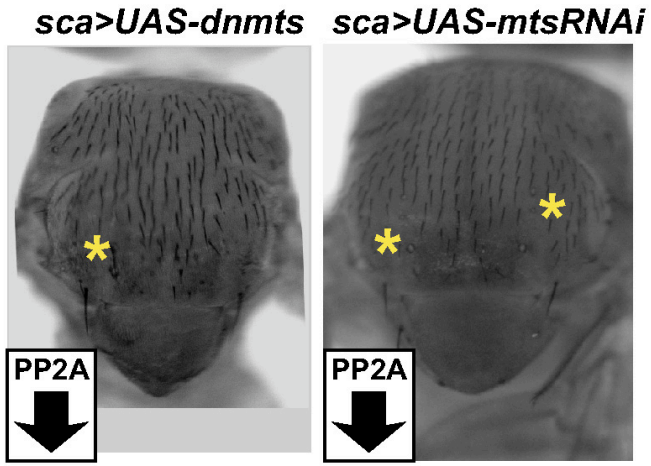

Figure 31: Ability of UAS-mtsRNAi and UAS-mtsDN constructs to elicit bristle abnormalities and comparison to UAS-Tik: A and B) UAS-construct encoding mts-RNAi was tested for its ability to elicit bristle defects. It was found that reducing PP2A levels elicited missing and split bristles (yellow) in the scutellum, unlike UAS-Tik, which elicited ectopic bristles. Therefore, reduced-PP2A levels and reduced-CK2 activity elicited similar phenotypes, both indicative of loss of $\mathrm{N}$ function. C) UAS-mtsDN construct was tested in parallel with mtsRNAi and elicited bristle loss and ectopic socket cells in a manner analogous to the RNAi construct. 
seen upon a reduction in the levels/activity of CK2. Furthermore, it has been found that the ability of ectopic $\mathrm{E}(\mathrm{spl}) \mathrm{M} 8$ to elicit bristle loss is neutralized by a reduction in the dosage of CK2. Based on these observations, studies were conducted to assess whether increased or decreased dosage of PP2A would alter $\mathrm{N}$ signaling.

A UAS-construct encoding mts-RNAi was tested for its ability to elicit bristle defects in otherwise wild type flies. Expression of the transgene was achieved with the Gal4 driver, G455.2, a driver that is active in proneural clusters that give rise to the scutellar macrochaetes. It was found that reducing PP2A levels elicited missing and split bristles in the scutellum. On the other hand, as previously shown UAS-Tik elicited ectopic bristles (Fig. 31A,B). Therefore, reduced-PP2A levels and reduced-CK2 activity elicited similar phenotypes, both indicative of loss of $\mathrm{N}$ function. Since there is a possibility that an RNAi construct can exhibit non-target effects, a UAS-mtsDN construct was tested in parallel with mtsRNAi. This $\mathrm{N}$-terminal truncated version of $\mathrm{mts}, \mathrm{mts}-\mathrm{DN}$, has previously been shown to reduce the activity of PP2A. Therefore, it was expected that mts-DN should behave in an analogous manner to the mtsRNAi construct and elicit missing bristles. As expected, expression of this construct with scaGal4 elicited bristle loss and ectopic socket cells in a manner analogous to the RNAi construct (Fig. 31C). Therefore, loss of CK2 and loss of PP2A elicit opposite bristle phenotypes.

Based on the opposite bristle phenotypes of mtsRNAi and Tik, I next assessed whether increased dosage of PP2A would mimic a CK2 loss of function. It was expected that increased PP2A activity should elicit a similar phenotype as reduced CK2 activity. Previously it was shown that expressing UAS-Tik elicited ectopic bristles. Therefore, for this analysis, UAS-mts and UAS-Tik constructs were expressed with scaGal4. It was found that 
$\mathbf{A}$
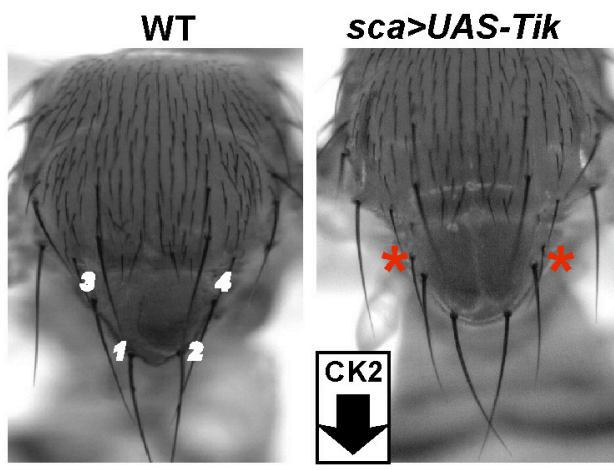

sca>UAS-mts

$\mathbf{B}$

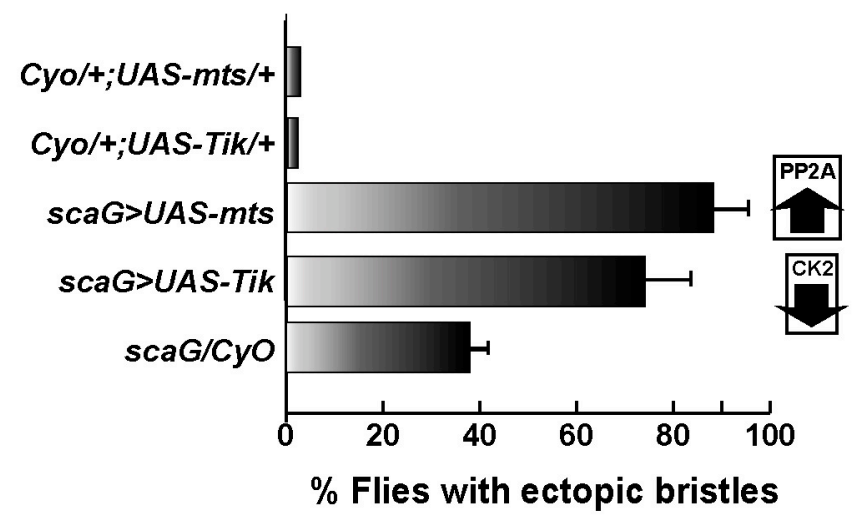

C

Average Scutellar/Thoracic Bristle counts

$\begin{array}{llc} & \text { scaGal4 } & \text { scaGal4 } \\ \text { Control } & 4.5 \pm 0.1 & 8.5 \pm 0.1 \\ \text { UAS-Tik } & 4.9 \pm 0.3 & -. .- \\ \text { UAS-mts } & -. .- & 10.8 \pm 0.3\end{array}$

Figure 32: Ability of UAS-mts construct to elicit bristle abnormalities and comparison

to UAS-Tik: A and B) UAS-construct encoding $\mathrm{mts}$ was tested for its ability to elicit bristle defects. Increased dosage of PP2A led to ectopic bristles, a phenotype that mimicked the effects of expression of a UAS-Tik construct 
increased dosage of PP2A led to ectopic bristles, a phenotype that mimicked the effects of expression of a UAS-Tik construct (Fig.32).

The effects of ectopic mts were also assessed via expression by $E(s p l) G a / 4$. This driver should recapitulate some of the expression patterns of the $E(s p l) C$. It was found increased dosage of PP2A led to a rough eye phenotype that was characterized by loss of ommatida, loss of the IOB's and ommatidial fusion (Fig. 33A). More importantly, overexpression of mts led to a wing nicking (notched) phenotype that has previously been described for mutations in $N$ (Fig. 33B).

\section{Ability to suppress bristle and eye phenotypes of $\boldsymbol{N}^{\text {spl: }}$}

Since CK2 and PP2A appeared to have opposite functions in N signaling, the effects of increased PP2A dosage were tested for its ability to suppress the retinal defects of $N^{s p l}$. As previously described, decreased dosage of CK2 suppressed the retinal defects of $N^{\text {spl }}$. In a similar manner increased dosage of PP2A led to a significant restoration of ommatidial numbers (Fig. 34). However, these effects appear to be slightly attenuated relative to expression of a UAS-CK2 $\alpha-M K$ construct. It is important to note that a PP2A loss of function did not lead to restored phasing of ommatidia (data not shown). The reasons for this remain to be clarified. 
A
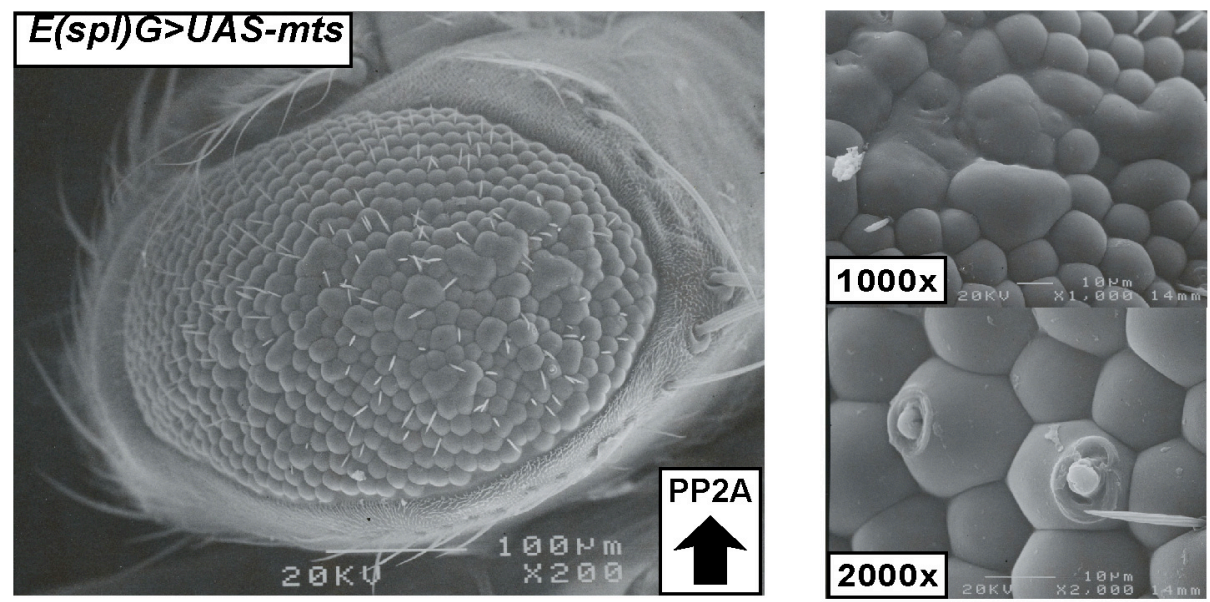

$\mathbf{B}$

WT

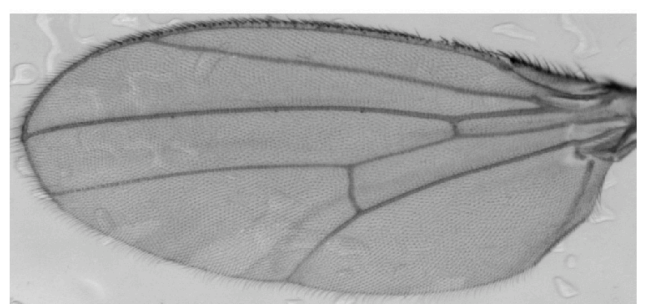

$E(s p l) G>U A S-m t s$

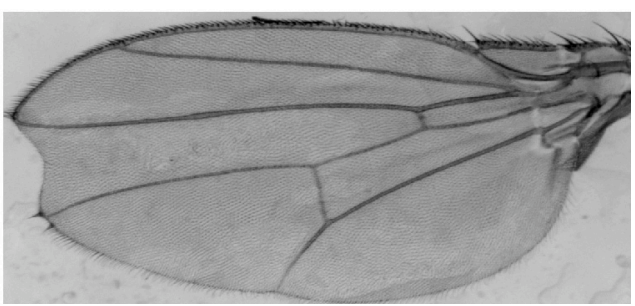

Figure 33: Ability of UAS-mts construct to elicit eye defects: A) UAS-construct encoding mts was tested for its ability to elicit eye defects. It was found increased dosage of PP2A led to a rough eye phenotype that was characterized by loss of ommatida, loss of the IOB's and ommatidial fusion. B) Increased dosage of PP2A of mts led to a wing nicking (notched) phenotype that has previously been described for mutations in $\mathrm{N}$. 
A
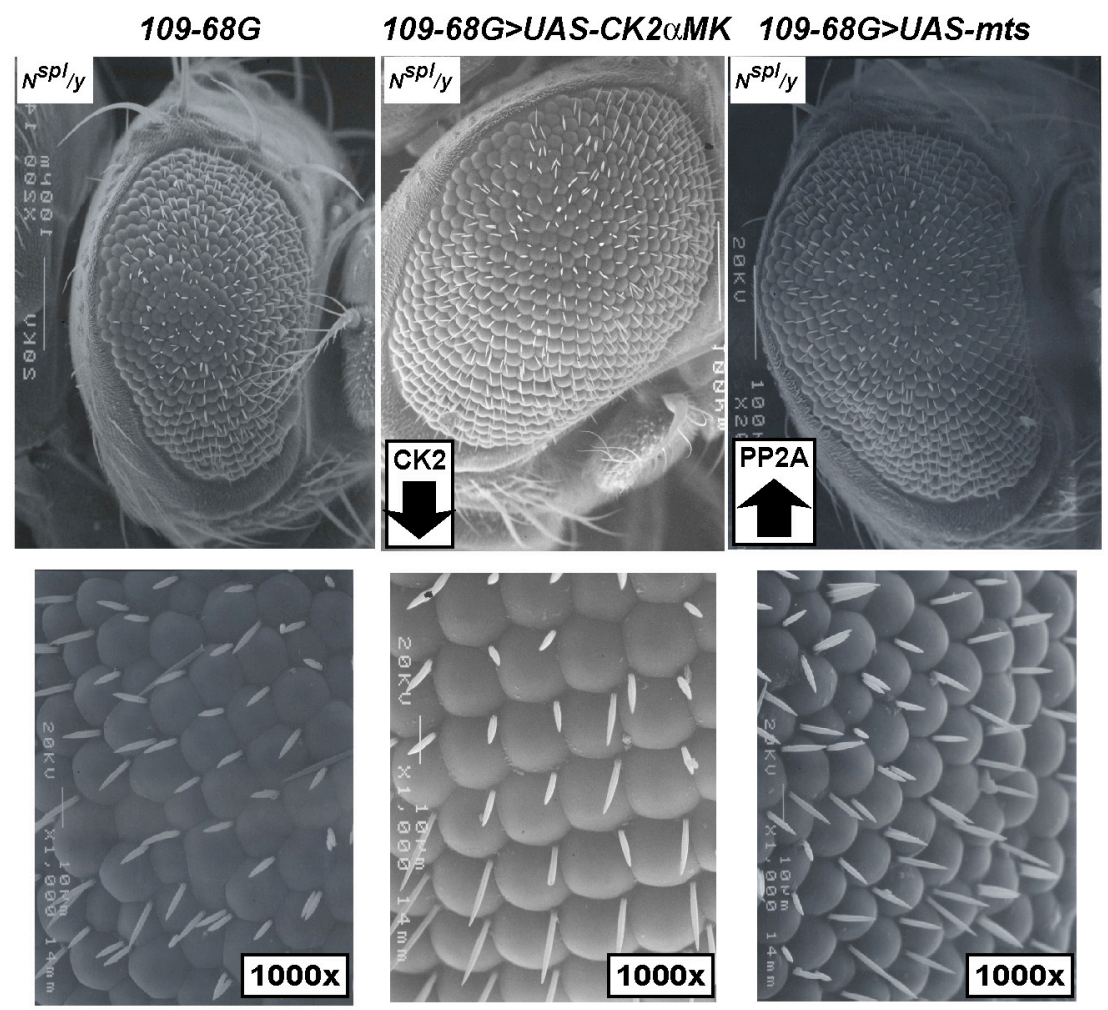

B

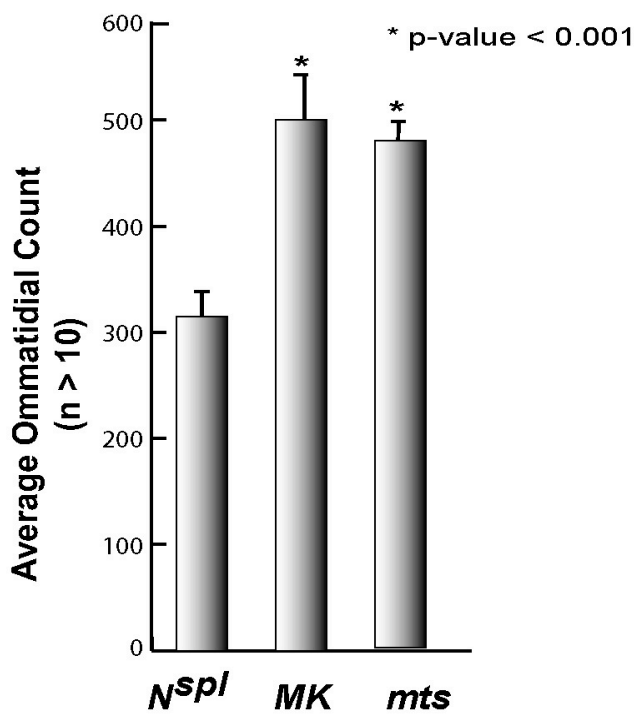

Figure 34: Ability of UAS-mts construct to suppress the eye phenotype of $\boldsymbol{N}^{s p} I$, and comparison to UAS-CK2 $\alpha-M K$. Increased dosage of PP2A led to a significant restoration of ommatidial numbers. 
CHAPTER 4.

CONCLUSIONS 
The studies described in this thesis might implicate a possible connection between CK2 and PP2A during N signaling in Drosophila eye and bristle neurogenesis. The role for $\mathrm{CK} 2$ in the regulation of $\mathrm{E}(\mathrm{spl})$ proteins and $\mathrm{N}$ signaling has been established based on the studies conducted in our laboratory. However, the identity and/or involvement of a cognate phosphatase is still unknown. It has also been speculated over the years that kinases act in supra-molecular complexes rather than act as isolated entities (Giot et al., 2003). This raises the possibility that CK2 might interact with other proteins such as phosphatases to orchestrate molecular events. PP2A is one of the candidates that have previously been shown to interact with CK2 and form stable complexes in vivo. However the molecular significance of this interaction remains elusive mostly due to the difficulty to find a suitable system to study this interaction.

Timekeeper (Tik), the dominant allele of $\mathrm{CK} 2 \alpha$, might provide a way to examine this relationship in vivo. As stated previously, Tik contains two amino acid substitutions, $\mathrm{M}^{161} \mathrm{~K}$ and $E^{165} \mathrm{D}$. The first substitution, $M K$, is positioned within the hydrophobic ATP binding pocket and disrupts the ATP binding. The second substitution, ED, is positioned away from the active site, but found in the region where PP2A binds to CK2. Therefore, Tik might be a 'double hit', on one side MK compromises the activity and on the other side ED perturbs the recruitment and/or regulation of PP2A. The analysis of Tik, thus, might provide a suitable system to study the relationship between CK2 and PP2A. To test this hypothesis, we generated two variants of CK2 that harbor either of these substitutions, and have analyzed them by a combinatorial approach. As expected, the MK variant displays minimal catalytic activity, whereas that with ED appears closer to the wild type. When tested in vivo, we find that misexpression of either the UAS-CK2 $\alpha M K$ or the UAS-CK $2 \alpha E D$ variant elicits ectopic bristles, which are classical $\mathrm{N}$ loss of function (LOF) phenotypes. We have also tested for the ability of these variants to suppress the neural defects of $N^{s p l}$. Previous studies 
suggested that reduced CK2 levels/activity suppresses the defects of this Notch gain of function (GOF) allele. We find that both MK and ED suppress the retinal defects of $N^{\text {spl }}$ with various degrees. Given that altered PP2A functions lead to clock and bristle defects, our results raise the possibility that Tik might, in fact, represent a 'double hit'. To further study the relationship between CK2 and PP2A, we have tested increased PP2A levels/activity (UAS-mts) and reduced PP2A levels/activity (UAS-mtsRNAi and UAS-dnmts). We have found that increased PP2A dosage elicits ectopic and missing bristles both indicative of a loss of $\mathrm{N}$ signaling. In addition, we find that increased dosage suppresses the rough and reduced eye phenotypes of $N^{s p l}$ flies, a result that is also associated with decreased CK2 levels/activity. On the other hand, reduced levels/activity of PP2A elicits bristle loss and ectopic socket cells, which are Notch gain of function (N GOF) phenotypes. Together, these results suggest that PP2A activity might attenuate, whereas CK2 augments, inhibitory $\mathrm{N}$ signaling. Given the previously described genetic and direct biochemical interactions between CK2 and E(spl)M8/M5/M7, PP2A might serve in an antagonistic manner to CK2. While this interpretation would be consistent with the data, it should be noted that a critical component of this nexus that has not yet been analyzed is the $E(s p l) C$, itself. Future efforts will therefore be required to better define the relationship between these regulatory proteins. Such studies might for example assess whether repression by ectopicly expressed $\mathrm{E}(\mathrm{spl})$ proteins can be modulated (augmented or antagonized) by changing the dosage of PP2A and/or CK2. 


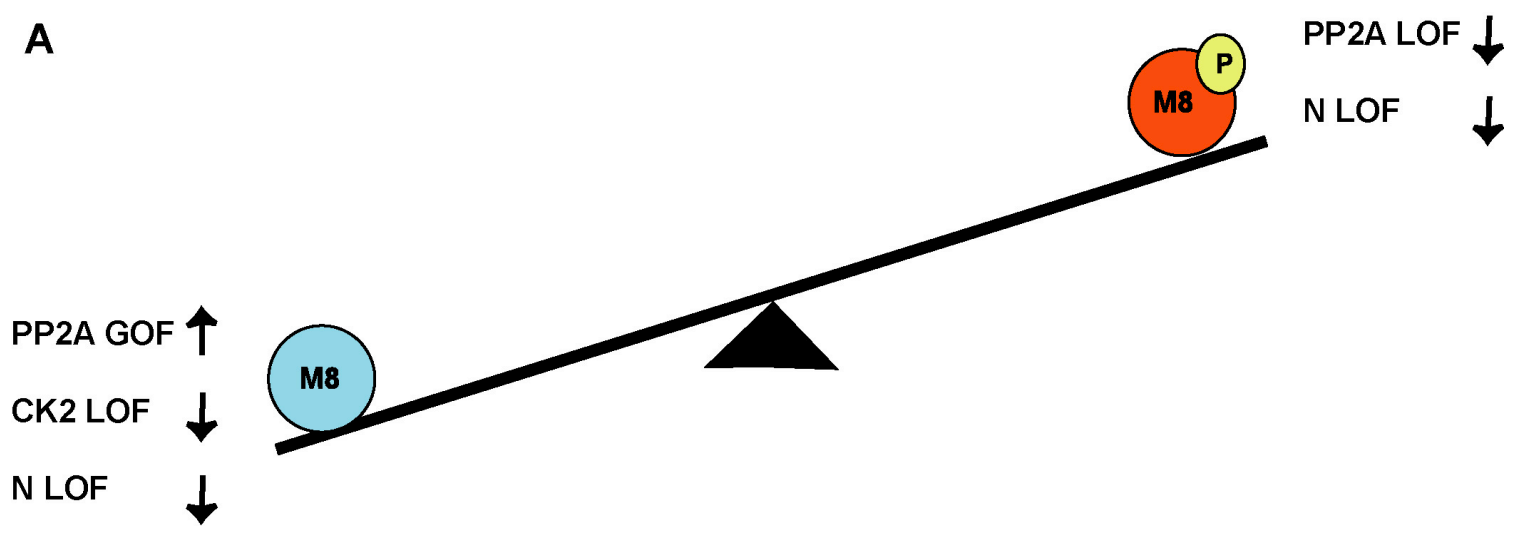

B

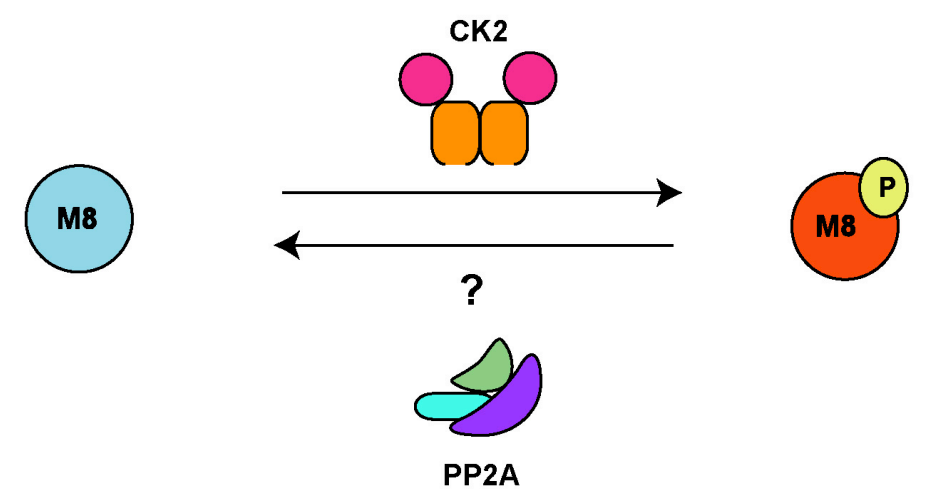

Figure 35: Potential antagonistic roles of CK2 and PP2A during $\mathbf{N}$ signaling. A)

Reduced CK2 activity/levels (CK2 LOF) and increased PP2A activity/levels (PP2A GOF) elicits phenotypes which have been shown in Notch loss of function (N LOF) mutants. On the other hand, reduced PP2A activity/levels (PP2A LOF) elicits phenotypes that have been found in Notch gain of function (N GOF) mutants. PP2A activity might attenuate, whereas CK2 augments, inhibitory $N$ signaling (hypophosphorylated M8 (blue) and hyperphosphorylated M8 (red)). B)Given the previously described genetic and direct biochemical interactions between $C K 2$ and $E(s p l) M 8 / M 5 / M 7, P P 2 A$ might serve in an antagonistic manner by dephosphorylating these proteins. 


\section{CHAPTER 5.}

REFERENCES 
Akten, B., Jauch, E., Genova, G. K., Kim, E. Y., Edery, I., Raabe, T., and Jackson, F. R. (2003). A role for CK2 in the Drosophila circadian oscillator. Nat Neurosc 6, 251-257. Allada, R. (2003). Circadian clocks: a tale of two feedback loops. Cell 112, 329-341. Allende, J. E., and Allende, C. C. (1995). Protein kinase CK2: An enzyme with multiple substrates and a puzzling regulation. FASEB J 9, 313-322.

Arroyo, J. D., and Hahn, W. C. (2005). Involvement of PP2A in viral and cellular transformation. Oncogene 24, 7746-7755.

Artavanis-Tsakonas, S., Matsuno, K., and Fortini, M. E. (1995). Notch Signalling. Science 268, 225-232.

Artavanis-Tsakonas, S., Rand, M. D., and Lake, R. J. (1999). Notch signaling: cell fate control and signal integration in development. Science 284, 770-776.

Ausubel, F. M., Brent, R., Kingston, R. E., Moore, D. D., Seidman, J. G., Smith, J. A., and Struhl, K., eds. (1989). Current Protocols in molecular biology. (New York, Wiley Interscience).

Baker, N. E., Yu, S., and Han, D. (1996). Evolution of proneural atonal expression during distinct regulatory phases in the developing Drosophila eye. Curr Biol 6, 1290-1301. Bandhakavi, S., McCann, R. O., Hanna, D. E., and Glover, C. V. (2002). A positive feedback loop between protein kinase CKII and Cdc37 promotes the activity of multiple protein kinases. J Biol Chem 278, 2829-2836.

Barford D, D. A., and MP. Egloff (1998). The structure and mechanism of protein phosphatases: insights into catalysis and regulation. Annu Rev Biophys Biomol Struct 27, 133-164.

Berger, J. (2004). Regulation of circdian rhythms. Journal of Applied Biomedicine 2, 131140.

Bidwai, A. P., Hanna, D. E., and Glover, C. V. C. (1992). Purification and characterization of Casein Kinase II (CKII) from DCKA1 DCKA2 S. cerevisiae rescued by Drosophila CKII subunits: The free catalytic subunit of casein kinase II is not toxic in vivo. J Biol Chem 267, 18790-18796.

Bidwai, A. P., Reed, J. C., and Glover, C. V. C. (1993). The phosphorylation of Calmodulin by the catalytic subunit of casein kinase II is inhibited by the regulatory subunit. Arch Biochem Biophys 300, 265-270.

Bidwai, A. P., Reed, J. C., and Glover, C. V. C. (1995). Cloning and disruption of CKB1, the gene encoding the 38-kDa beta subunit of Saccharomyces cerevisiae casein kinase II 
(CKII). Deletion of CKII regulatory subunits elicits a salt-sensitive phenotype. J Biol Chem 270, 10395-10404.

Bidwai, A. P., Zhao, W. F., and Glover, C. V. C. (1999). A gene located at 56F1-2 in Drosophila melanogaster encodes a novel metazoan beta-like subunit of casein kinase II. Mol Cell Biol Res Comm 1, 21-28.

Blaumuller, C. M., and Artavanis-Tsakonas, S. (1997). Comparative aspects of Notch signaling in lower and higher eukaryotes. Perspect Dev Neurobiol 4, 325-343.

Boldyreff, B., and Issinger, O. G. (1997). A-Raf kinase is a new interacting partner of protein kinase CK2 beta subunit. FEBS Lett 403, 197-199.

Bosc, D. G., Graham, K. C., Saulnier, R. B., Zhang, C., Prober, D., Gietz, R. D., and Litchfield, D. W. (2000). Identification and Characterization of CKIP-1, a Novel Pleckstrin Homology Domain-containing Protein That Interacts with Protein Kinase CK2. J Biol Chem 275, 14295-14306.

Bosc, D. G., Slominski, E., Sichler, C., and Litchfield, D. W. (1995). Phosphorylation of casein kinase II by p34 cdc2. J Biol Chem 270, 25872-25878.

Bose, A., Kahali, B., Zhang, S., Lin, J.-M., Allada, R., Karandikar, U., and Bidwai, A. (2006). Drosophila CK2 regulates lateral-inhibition during eye and bristle development. Mech Dev 123, 649-664.

Bourbon, H. M., Martin-Blanco, E., Rosen, D., and Kornberg, T. B. (1995). Phosphorylation of the Drosophila Engrailed protein at a site outside its homeodomain enhances DNA binding. J Biol Chem 270, 11130-11139.

Brand, A. H., and Perrimon, N. (1993). Targeted gene expression as a means of altering cell fates and generating dominant phenotypes. Development 118, 401-415.

Bray, S. (1998). A notch affair. Cell 93, 499-503.

Burnett, G., and Kennedy, E. P. (1954). The enzymatic phosphorylation of proteins. J Biol Chem 211, 969-980.

Cadigan (1997). Wnt signaling: a common theme in animal development. Genes \& Dev 11, 3286-3305.

Chantalat, L., Leroy, D., Filhol, O., Nueda, A., Benitez, M. J., Chambaz, E. M., Cochet, C., and Dideberg, O. (1999). Crystal structure of the human protein kinase CK2 regulatory subunit reveals its zinc finger-mediated dimerization. EMBO J 18, 2930-2940.

Chen, G., and Courey, A. J. (2000). Groucho/TLE family proteins and transcriptional repression. Gene 249, 1-16. 
Cho, U. S., Morrone, S., Sablina, A. A., Arroyo, J. D., Hahn, W. C., and Xu, W. (2007). Structural Basis of PP2A Inhibition by Small t Antigen. PLoS Biol 5, e202.

Cho, U. S., and Xu, W. (2007). Crystal structure of a protein phosphatase 2A heterotrimeric holoenzyme. Nature 445, 53-57.

Ciccarelli FD, A. A., and S. Alberti (2000). Large and diverse numbers of human diseases with HIKE mutations. Hum Mol Genet 9, 1001-1007.

Cochet, C., and Chambaz, E. M. (1983). Oligomeric structure and catalytic activity of G type casein kinase. J Biol Chem 258, 1403-1406.

Coghlan, V. M., Perrino, B. A., Howard, M., Langeberg, L. K., Hicks, J. B., Gallatin, W. M., and Scott, J. D. (1995). Association of protein kinase A and protein phosphatase 2B with a common anchoring protein. Science 267, 108-111.

Dawson, S. R., Turner, D. L., Weintraub, H., and Parkhurst, S. M. (1995). Specificity for the hairy/enhancer of split basic helix-loop-helix (bHLH) proteins maps outside the bHLH domain and suggests two separable modes of transcriptional repression. Mol Cell Biol 15, 6923-6931.

Delidakis, C., and Artavanis-Tsakonas, S. (1991). The enhancer of split [E(spl)] locus of Drosophila encodes seven independant helix-loop-helix proteins. Proc Natl Acad Sci USA 89, 8731-8735.

Donella-Deana, A., Cesaro, L., Sarno, S., Brunati, A. M., Ruzzene, M. and L. A., Pinna (2001). Autocatalytic tyrosine-phosphorylation of protein kinase CK2 alpha and alphah subunits : implication of Tyr182. Biochem 357, 563-567.

Draetta, G. (1990). Cell cycle control in eukaryotes: Molecular mechanisms of cdc2 activation. Trends Biochem Sci 15, 378-387.

Duffy, J. B. (2002). GAL4 System in Drosophila: A Fly Geneticist's Swiss Army Knife. Genesis 34, 1-15.

Edelman, A. M., Blumenthal, D. K., and Krebs, E. G. (1987). Protein serine/threonine kinases. Ann Rev Biochem 56, 567-613.

Filhol, O., Baudier, J., Delphin, C., Loue-Machenbach, P., Chambaz, E. M., and Cochet, C. (1992). Casein kinase II and the tumor suppressor protein p53 associate in a molecular complex that is negatively regulated by p53 phosphorylation. J Biol Chem 267, 2057720583.

Flores, G. V., Duan, H., Yan, H., Nagaraj, R., Fu, W., Zou, Y., Noll, M., and Banerjee, U. (2000). Combinatorial signaling in the specification of unique cell fates. Cell 103, 75-85. 
Gao, Y., and Wang, H. Y. (2006). Casein kinase 2 Is activated and essential for Wnt/betacatenin signaling. J Biol Chem 281, 18394-18400.

Ghavidel, A., and Schultz, M. C. (2001). TATA binding protein-associated CK2 transduces DNA damage signals to the RNA polymerase II transcriptional machinery. Cell 106, 575584.

Giot, L., Bader, J. S., Brouwer, C., Chaudhuri, A., Kuang, B., Li, Y., Hao, Y. L., Ooi, C. E., Godwin, B., Vitols, E., et al. (2003). A protein interaction map of Drosophila melanogaster. Science 302, 1727-1736.

Glover, C. V. C. (1998). On the physiological role of casein kinase II in Saccharomyces cerevisiae. Prog Nuc Acid Res \& Mol Biol 59, 95-133.

Glover, C. V. C., Bidwai, A. P., and Reed, J. C. (1994). Structure and function of Saccharomyces cerevisiae casein kinase II. Cell Mol Biol Res 5/6, 481-488.

Glover, C. V. C., Shelton, E. R., and Brutlag, D. L. (1983). Purification and characterization of a type II casein kinase from Drosophila melanogaster. J Biol Chem 258, 3258-3256.

Goldberg, Y. (1999). Protein phosphatase 2A: who shall regulate the regulator? Biochem Pharmacol 57, 321-328.

Goldsmith, C. a. (1998). How MAP kinases are regulated. J Biol Chem 270, 14843-14846. Groves MR, H. N., Turowski P, Hemmings BA, and D.Barford (1999). The structure of the protein phosphatase 2A PR65/A subunit reveals the conformation of its 15 tandemly repeated HEAT motifs. Cell 96, 99-110.

Gurland G, G. G. (1993). Protein phosphatase inhibitors induce the selective breakdown of stable microtubules in fibroblasts and epithelial cells. Proc Natl Acad Sci U S A 90, 88278831.

Hanna, D. E., Rethinaswamy, A., and Glover, C. V. C. (1995). Casein kinase II is required for cell cycle progression during $\mathrm{G} 1$ and G2/M in Saccharomyces cerevisiae. J Biol Chem 270, 25905-25914.

Harper, J. W., Adami, G. R., Wei, N., Keyomarski, K., and Elledge, S. (1993). The p21 cdkinteracting protein cip1 is a potent inhibitor of $\mathrm{G} 1$ cyclin-dependant kinases. Cell $75,805-$ 816.

Hathaway, G. M., Lubben, T. H., and Traugh, J. A. (1980). Inhibition of casein kinase II by heparin. J Biol Chem 255, 8038-8041.

Hathaway, G. M., and Traugh, J. A. (1984). Interaction of polyamines and magnesium with casein kinase II. Arch Biochem Biophys 233, 133-138. 
Haystead TA, S. A., Carling D, Honnor RC, Tsukitani Y, Cohen P, Hardie DG. (1989).

Effects of the tumour promoter okadaic acid on intracellular protein phosphorylation and metabolism. Nature 337, 78-81.

Henriksson, M. a. L., B. (1996). Proteins of the Myc network: essential regulators of cell growth and differentiation. Adv Cancer Res 68, 109-182.

Heriche, J. K., Lebrin, F., Rabilloud, T., Leroy, D., Chambaz, E. M., and Goldberg, Y. (1997).

Regulation of protein phosphatase $2 \mathrm{~A}$ by direct interaction with casein kinase 2alpha.

Science 276, 952-955.

Hsiung, F., and Moses, K. (2002). Retinal development in Drosophila: specifying the first neuron. Human Molecular Genetics 11, 1207-1214.

Hunter, T. (1995). Protein kinases and phosphatases: The yin and yang of protein phosphorylation and signaling. Cell 80, 225-236.

Jaffe, L., Ryoo, H.-D., and Mann, R. S. (1997). A role for phosphorylation by casein kinase II in modulating Antennapedia activity in Drosophila. Genes \& Develop 11, 1327-1340.

Janssens, V., and Goris, J. (2001). Protein phosphatase 2A: a highly regulated family of serine/threonine phosphatases implicated in cell growth and signalling. Biochem $\mathrm{J} 353,417-$ 439.

Jauch, E., Melzig, J., Brkulj, M., and Raabe, T. (2002). In vivo functional analysis of Drosophila protein kinase casein kinase 2 (CK2) beta-subunit. Gene 298, 29-39. Jiang, Y. (2006). Regulation of the cell cycle by protein phosphatase 2A in Saccharomyces cerevisiae. Microbiol Mol Biol Rev 70, 440-449.

Karandikar, U., Anderson, S., Mason, N., Trott, R. L., Bishop, C. P., and Bidwai, A. P. (2003). The Drosophila SSL gene is expressed in larvae, pupae, and adults, exhibits sexual dimorphism, and mimics properties of the b subunit of casein kinase II. Biochem Biophys Res Commun 301, 941-947.

Karandikar, U., Trott, R. L., Yin, J., Bishop, C. P., and Bidwai, A. P. (2004). Drosophila CK2 regulates eye morphogenesis via phosphorylation of E(spl)M8. Mech Develop 121, 273-286. Knighton, D. R., Zheng, J., Ten-Eyck, L. F., Xuong, N. H., Taylor, S. S., and Sowadski, J. M. (1991). Structure of a Peptide Inhibitor Bound to the Catalytic Subunit of Cyclic Adenosine Monophosphate-Dependant Protein Kinase. Science 253, 414-420.

Kobe B, G. T., Horne J, Jennings IG, Scotney PD, and T. Teh (1999). Turn up the HEAT. Structure 7, 91-97.

Krebs, E. G. (1986). The enzymology of control by phosphorylation, Vol $17,3^{\text {rd }}$ edn, Academic Press). 
Krebs, E. G., Graves, D. J., and Fischer, E. H. (1959). Factors affecting the activity of muscle phosphorylase b kinase. J Biol Chem 234, 2867-2873.

Kudlicki, W. N., Szyszka, R., and Gasior, E. (1984). A cytoplasmic cyclic nucleotideindependent casein kinase II from Saccharomyces cerevisiae. Biochim Biophys Acta 784, 102-107.

Kuenzel, E. A., Mulligan, J. A., Sommercorn, J., and Krebs, E. G. (1987). Substrate specificity determinants for casein kinase II as deduced from studies with synthetic peptides. J Biol Chem 262, 9136-9140.

Laemmli, U. K. (1970). Cleavage of structural proteins during the assembly of the head of bacteriophage T4. Nature (London) 227, 680-685.

Lai, E. C. (2004). Notch signaling: control of cell communication and cell fate. Development 131, 965-973.

Lebrin F, B. L., Rabilloud T, Chambaz EM, and Y. Goldberg (1999). CK2alpha-protein phosphatase 2A molecular complex: possible interaction with the MAP kinase pathway. Mol Cell Biochem 191, 207-212.

Li, Y., Lei, L., Irvine, K. D., and Baker, N. E. (2003). Notch activity in neural cells triggered by a mutant allele with altered glycosylation. Development 130, 2829-2840.

Ligoxygakis, P., Yu, S. Y., Delidakis, C., and Baker, N. E. (1998). A subset of Notch functions during Drosophila eye development require $\mathrm{Su}(\mathrm{H})$ and $E(\mathrm{spl})$ gene complex. Develop 125, 2893-2900.

Lin, J. M., Kilman, V. L., Keegan, K., Paddock, B., Emery-Le, M., Rosbash, M., and Allada, R. (2002). A role for casein kinase 2alpha in the Drosophila circadian clock. Nature 420, 816-820.

Litchfield, D. (2001). Functional specialization of CK2 isoforms and characterization of isoform-specific binding partners. Molecular and Cellular Biochemistry 227, 21-29.

Litchfield, D. W. (2003). Protein kinase CK2: structure, regulation and role in cellular decisions of life and death. Biochem J 369, 1-15.

Liu, Z. P., Galindo, R. L., and Wasserman, S. A. (1997). A role for CKII phosphorylation of the cactus PEST domain in dorsoventral patterning of the Drosophila embryo. Genes Dev 11, 3413--3422.

Longin S, Z. K., Louis JV, Dilworth S, Goris J, Janssens V. (2007). Selection of protein phosphatase $2 \mathrm{~A}$ regulatory subunits is mediated by the $\mathrm{C}$ terminus of the catalytic Subunit. $\mathrm{J}$ Biol Chem 282, 26971-26980. 
Ma, C., and Moses, K. (1995). Wingless and patched are negative regulators of the morphogenetic furrow and can affect tissue polarity in the developing Drosophila compound eye. Development 121, 2279-2289.

MacKay, V. a. M., T. R. (1974). Mutations affecting sexual conjugation and related processes in Saccharomyces cerevisiae. I. Isolation and phenotypic characterization of nonmating mutants. Genetics 76, 255-271.

Mayer-Jaekel RE, O. H., Gomes R, Sunkel CE, Baumgartner S, Hemmings BA, Glover DM. (1993). he $55 \mathrm{kd}$ regulatory subunit of Drosophila protein phosphatase 2A is required for anaphase. Cell 72, 621-633.

Meggio, F., Brunati, A. M., and Pinna, L. A. (1983). Autophosphorylation of type 2 casein kinase TS at both its alpha and beta subunits. FEBS Lett 160, 203-208.

Meggio, F., and Pinna, L. A. (2003). One-thousand-and-one substrates of protein kinase CK2. FASEB J 17, 349-368.

Meier, U. T., and Blobel, G. (1992). Nopp 140 shuttles on tracks between nucleolus and cytoplasm. Cell 70, 127-138.

Miller, J. H. (1972). Experiments in molecular genetics. In (New York, Cold Spring Harbor), pp. 352-355.

Miyata, Y., and Yahara, I. (1992). The 90-kDa heat shock protein, HSP90, binds and protects casein kinase II from self aggregation and enhances its kinase activity. J Biol Chem 267, 7042-7047.

Mumby, M. (2001). A new role for protein methylation: switching partners at the phosphatase ball. Sci STKE 2001, pe. 1.

Mumby MC, W. G. (1993). Protein serine/threonine phosphatases: structure, regulation, and functions in cell growth. Physiol Rev 73, 673-699.

Mumm, J. S., and Kopan, R. (2000). Notch signaling: From the outside in. Dev Biol 228, 151-165.

Niefind, K., Guerra, B., Ermakowa, I., and Issinger, O. G. (2001). Crystal structure of human protein kinase CK2: insights into basic properties of the CK2 holoenzyme. EMBO J 20, 5320-5331.

Oelgeschlager, M., Krieg, J., Luscher-Firzla?, J. M. and Luscher, B. (1995). Casein kinase II phosphorylation site mutations in c-Myc affect DNA binding and transcriptional cooperivity with NF-M. Mol Cell Biol 15, 5966-5974.

Olsen, B. B., Petersen, J., and Issinger, O. G. (2006). BID, an interaction partner of protein kinase CK2alpha. Biol Chem 387, 441-449. 
Olsten ME, W. J., and DW Litchfield (2005). CK2 interacting proteins: emerging paradigms for CK2 regulation? Mol Cell Biochem 274, 115-124.

Phillip Kaldis. (1999). The cdk-activating kinase (CAK): from yeast to mammals. Cell Mol Life Sci 55, 284-296.

Packman, L. C., Kubota, K., Parker, J., and Gay, N. J. (1997). Casein kinase II phosphorylates Ser468 in the PEST domain of the Drosophila IkappaB homologue cactus. FEBS Lett 400, 45-50.

Padmanabha, R., Chen-Wu, J. L. P., Hanna, D. E., and Glover, C. V. C. (1990). Isolation, sequencing, and disruption of the yeast CKA2 gene: Casein kinase II is essential for viability in Saccharomyces cerevisiae. Mol Cell Biol 10, 4089-4099.

Paroush, Z., Finley, R. L., Kidd, T., Wainwright, S. M., Ingham, P. W., Brent, R., and IshHorowcz, D. (1994). Groucho is required for Drosophila neurogenesis, segmentation, and sex determination and interacts directly with hairy related bHLH proteins. Cell 79, 805-815. Pepperkok, R., Lorenz, P., Ansorge, W., and Pyerin, W. (1994). Casein kinase II is required for transition of G0/G1, early G1, and G1/S phases of the cell cycle. J Biol Chem 269, 69866991.

Pinna, L. A. (1990). Casein kinase 2: an 'eminence grise' in cellular regulation? Biochim Biophys Acta 1054, 267-284.

Pinna, L. A. (1994). A historical view of protein kinase CK2. Cell Mol Biol Res 40, 383-390.

Pinna, L. A. (2002). Protein kinase CK2: a challenge to canons. J Cell Sci 115, 3873-3878.

Polakis, P. (2000). Wnt signaling and cancer. Genes Dev 14, 1837-1851.

Price, J. V., Savenye, E. D., Lum, D., and Breitkreutz, A. (1997). Dominant enhancers of Egfr in Drosophila melanogaster: genetic links between the Notch and Egfr signaling pathways. Genetics 147, 1139-1153.

Rasmussen, T., ISkjøth, I. H. E., Jensen, H. H., Niefind, K., Boldyreff, B., and Issinger, O. G. (2005). Biochemical characterization of the recombinant human Drosophila homologues Timekeeper and Andante involved in the Drosophila circadian oscillator. Mol Cell Biochem 274, 151-161.

Ratcliffe MJ, I. K., Sokol SY. (2000). A positive role for the PP2A catalytic subunit in Wnt signal transduction. J Biol Chem 275, 35680-35683.

Rethinaswamy, A., Birnbaum, M. J., and Glover, C. V. C. (1998). Temperature-sensitive mutations of the CKA1 gene reveal a role for casein kinase II in maintanance of cell polarity in Saccharomyces cerevisiae. J Biol Chem 273, 5869-5877.

Rimington, C. (1926). Phosphorylation of Proteins. Biochem 21, 272-281. 
Rivera T, a. A. L. (2006). Shugoshin and PP2A, shared duties at the centromere. Bioassays 28, 775-779.

Russo, G. L., Vandenberg, M. T., Yu, J. Y., Bae, Y. S., Franza, B. R., and Marshak, D. R. (1992). Casein kinase II phosphorylates p34cdc2 kinase in G1 phase of the HeLa cell division cycle. J Biol Chem 267, 20317-20325.

Ruvolo PP, D. X., Ito T, Carr BK, May WS. (1999). Ceramide induces Bcl2 dephosphorylation via a mechanism involving mitochondrial PP2A. J Biol Chem 274, 2029620300.

Ruzzene, M., Penzo, D., and Pinna, L. A. (2002). Protein kinase CK2 inhibitor 4,5,6,7tetrabromobenzotriazole (TBB) induces apoptosis and caspase-dependent degradation of haematopoietic lineage cell-specific protein 1 (HS1) in Jurkat cells. Biochem J 364, 41-47. Sathyanarayanan, S., Zheng, X., Xiao, R., and Sehgal, A. (2004). Posttranslational regulation of Drosophila PERIOD protein by protein phosphatase 2A. Cell 116, 603-615. Solomon, M. J., Glotzer, M., Lee, T. H., Philippe, M., and Kirschner, M. W. (1990). Cyclin activation of $\mathrm{p} 34^{\mathrm{cdc} 2}$. Cell $63,1013-1024$.

Sontag, E., Fedorov, S., Kamibayashi, C., Robbins, D., Cobb, M., and Mumby, M. (1993). The interaction of SV40 small tumor antigen with protein phosphatase $2 \mathrm{~A}$ stimulates the map kinase pathway and induces cell proliferation. Cell 75, 887-897.

Taylor, S. S. (1989). cAMP-dependent protein kinase: Model for an enzyme family. J Biol Chem 264, 8443-8446.

Tournier-Lasserve, A. J. a. E. (1998). Notch signaling pathway and human disease. Semin Cell and Dev Biol 9, 619-625.

Tuazon, P. T., and Traugh, J. A. (1990). Casein kinase I and II - multipotential serine protein kinases: Structure, function and regulation. In Advances in second messenger and phosphoprotein research., P. Greengard, and G. A. Robinson, eds. (New York, Raven Press), pp. 123-164.

Tuazon, P. T., and Traugh, J. A. (1991). Advances in second messenger and phosphoprotein research. In (New York, Raven Press), pp. 123-164.

Uemura T, S. K., Togashi S, Takeichi M. (1993). Mutation of twins encoding a regulator of protein phosphatase $2 \mathrm{~A}$ leads to pattern duplication in Drosophila imaginal discs. Genes \& Dev 7, 429-440.

Verde F, M. J., Nurse P. (1995). Fission yeast cell morphogenesis: identification of new genes and analysis of their role during the cell cycle. J Cell Biol 131, 1529-1538. 
Wang Y, N. T. (2005). Phosphatase 2A negatively regulates mitotic exit in Saccharomyces cerevisiae. Mol Biol Cell 17, 80-89.

Wassarman, D. A., Solomon, N. M., Chang, H. C., Karim, F. D., Therrien, M., and Rubin, G. M. (1996). Protein phosphatase 2A positively and negatively regulates Ras1-mediated photoreceptor development in Drosophila. Genes Dev 10, 272-278.

Xing, Y., Xu, Y., Chen, Y., Jeffrey, P. D., Chao, Y., Lin, Z., Li, Z., Strack, S., Stock, J. B., and Shi, Y. (2006). Structure of protein phosphatase $2 A$ core enzyme bound to tumor-inducing toxins. Cell 127, 341-353.

Xu, Y., Xing, Y., Chen, Y., Chao, Y., Lin, Z., Fan, E., Yu, J. W., Strack, S., Jeffrey, P. D., and Shi, Y. (2006). Structure of the protein phosphatase 2A holoenzyme. Cell 127, 1239-1251. Xu Z, W. B. (2000). The B56alpha regulatory subunit of protein phosphatase 2A is a target for regulation by double-stranded RNA-dependent protein kinase PKR. Mol Cell Biol 20, 5285-5299.

Yarden, Y., and Ullrich, A. (1988). Growth factor receptor tyrosine kinases. Ann Rev Biochem 57, 443-478.

Zhang, C.-K., Vilk, G., Canton, D. A., and Litchfield, D. W. (2002). Phosphorylation regulates the stability of the regulatory CK2beta subunit. Oncogene $21,3754-3764$. 


\section{APPENDIX A}

\begin{tabular}{|c|c|c|}
\hline Primer Name & Sequence (5'-3') & Description \\
\hline DMABAMPK & $\begin{array}{l}\text { t agg atc cgc aac atg aca ctt cct agt } \\
\text { gcg gct }\end{array}$ & $\begin{array}{l}\text { Forward primer to introduce } \\
\text { BamH1 site to the dCK2 } \alpha \text { ORF }\end{array}$ \\
\hline DMA-XH2PK & $\begin{array}{l}\text { gcg ctc gag tta ttg ctg att att ggg att } \\
\text { cat } t\end{array}$ & $\begin{array}{l}\text { Reverse primer to introduce Xho1 } \\
\text { site to the dCK2 } \alpha \text { ORF }\end{array}$ \\
\hline Tik-P1 & $\begin{array}{l}\text { gta aag ccc cac aat gtt aag ata gat } \\
\text { cac gac aat cga aat tgc gcc }\end{array}$ & $\begin{array}{l}\text { Forward SDM primer for } \\
\text { dCK } 2 \alpha-\text { Tik }\end{array}$ \\
\hline Tik-P2 & $\begin{array}{l}\text { ggc gca att ttt cga ttg tcg tga tct atc } \\
\text { tta aca ttg tgg ggg ctt tac }\end{array}$ & $\begin{array}{l}\text { Reverse SDM primer for } \\
\text { dCK2 } \alpha \text {-TiK }\end{array}$ \\
\hline Tik-MKP1 & $\begin{array}{l}\text { gta aag ccc cac aat gtt aag ata gat } \\
\text { cac gaa aaa tcg aaa att gcg cc }\end{array}$ & $\begin{array}{l}\text { Forward SDM primer for } \\
\text { dCK2 } \alpha-M K\end{array}$ \\
\hline Tik-MKP2 & $\begin{array}{l}\text { ggc gca att ttc gat ttt cgt gat cta tct } \\
\text { taa cat tgt ggg gct tta c }\end{array}$ & $\begin{array}{l}\text { Reverse SDM primer for } \\
\text { dCK } 2 \alpha-M K\end{array}$ \\
\hline Tik-EDP1 & $\begin{array}{l}\text { gta aag ccc cac aat gtt atg ata gat } \\
\text { cac gac aat cga aaa ttg cgc c }\end{array}$ & $\begin{array}{l}\text { Forward SDM primer for } \\
\text { dCK2 } \alpha-E D\end{array}$ \\
\hline Tik-EDP2 & $\begin{array}{l}\text { ggc gca att ttc gat tgt cgt gat cta tca } \\
\text { taa cat tgt ggg get tta c }\end{array}$ & $\begin{array}{l}\text { Reverse SDM primer for } \\
\text { dCK2 } \alpha-E D\end{array}$ \\
\hline dCK2E2-P1 & $\begin{array}{l}\text { gga cca acc gac tga gaa cgg aat } \\
\text { cag c }\end{array}$ & Forward primer for dCK2 $\alpha$-Exon3 \\
\hline dCK2E2-P2 & $\begin{array}{l}\text { ggc ttc tat ccc aaa ctc gaa tta cag } \\
\text { tat tca tc }\end{array}$ & Reverse primer dCK2 $\alpha$-Exon3 \\
\hline
\end{tabular}

Table 3: Primers used during this study: Multiple set of primers were employed throughout this study to introduce either point mutations or specified deletions as well as for routine PCR synthesis. 